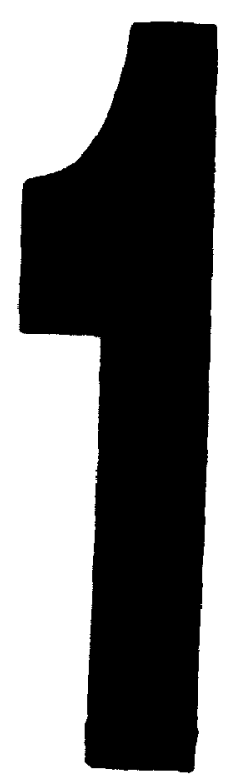

PM-1 3K"

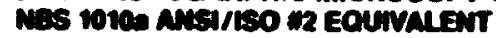

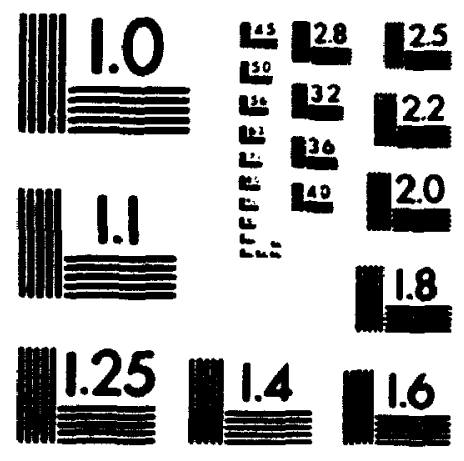

PRECHSIONm RESOLUTION TARCETS 
National Libray

of Canada

Acquisitions and Bibliographic Senvices Branch

395 wetingen sired Orewa. Oritero KiA ONi
Bifliotheque nationale

du Canada

Direction des acquisitions et

des senvices bibliographiques

395. na watingon

Onive (Ontaio)

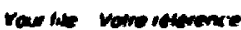

Orw menes

AVIS

The quality of this microform is heavily dependent upon the quality of the original thesis submitted for microfilming. Every effort has been made to ensure the highest quality of reproduction possible.

If pages are missing, contact the university which granted the degree.

Some pages may have indistinct print especially if the original pages were typed with a poor typewriter ribbon or if the university sent us an inferior photocopy.
La qualité de cette microforme dépend grandement de la qualité de la these soumise au microfilmage. Nous avons tout fait pour assurer une qualité supérieure de reproduction.

S'il manque des pages, veuillez communiquer avec l'université qui a conféré le grade.

La qualité d'impression de certaines pages peut laisser à désirer, surtout si les pages originales ont été dactylographiées à l'aide d'un ruban usé ou si l'université nous a fait parvenir une photocopie de qualité inférieure.

La reproduction, même partielle, de cette microforme est soumise a la Loi canadienne sur le droit d'auteur, SRC 1970, c. C-30, et ses amendements subséquents.
Reproduction in full or in part of this microform is governed by the Canadian Copyright Act, R.S.C. 1970, c. C-30, and subsequent amendments. 


\title{
Integrating Process Modeling and Metrics Support for Object Oriented Software Development
}

\author{
by \\ Craig Robert Hayashi \\ A thesis submitted to \\ the Faculty of Graduate Studies and Research \\ in partial fulfillment of \\ the requirements for the degree of \\ Master of Computer Science \\ School of Cumputer Science \\ Carleton University \\ Ottawa, Ontario \\ November 10, 1994 \\ copyright \\ 1994, Craig Robert Hayashi
}


National Libray

of Canada

Acquisitions and

Bibliographic Senvices Branch

395 Wetingron Streer

Ourma. Orierio

Kinon
Bibliotheque nationale du Canada

Direction des acquisitions et des senvices bibliographiques

395. ne Weungton

Ontwa (Ontano)

KIA ONA
THE AUTHOR HAS GRANTED AN IRREVOCABLE NON-EXCLUSIVE LICENCE ALLOWING THE NATIONAL LIBRARY OF CANADA TO REPRODUCE, LOAN, DISTRIBUTE OR SELL COPIES OF HIS/HER THESIS BY ANY MEANS AND IN ANY FORM OR FORMAT, MAKING THIS THESIS AVAILABLE TO INTERESTED PERSONS.
L'AUTEUR A ACCORDE UNE LICENCE IRREVOCABLE ET NON EXCLUSIVE PERMETTANT A LA BIBLIOTHEQUE NATIONALE DU CANADA DE REPRODUIRE, PRETER, DISTRIBUER OU VENDRE DES COPIES DE SA THESE DE QUELQUE MANIERE ET SOUS QUELQUE FORME QUE CE SOIT POUR METTRE DES EXEMPLAIRES DE CETTE THESE A LA DISPOSITION DES PERSONNE INTERESSEES.
THE AUTHOR RETAINS OWNERSHIP

OF THE COPYRIGHT IN HIS/HER THESIS. NEITHE? THE THESIS NOR SUBSTANTIAL EXTRACTS FROM IT MAY BE PRINTEO OR OTHERWISE REPRODUCED WITHOUT HIS/HER PERMISSION
L'AUTEUR CONSERVE LA PROPRIETE DU DROIT D'AUTEUR QUI PROTEGE SA THESE. NI LA THESE NI DES EXTRAITS SUBSTANTIELS DE CELLECI NE DOIVENT ETRE IMPRIMES OU AUTREMENT REPRODUITS SANS SON AUTORISATION.

ISBN $\quad 0-612-03002-4$ 
Nome

Hayashi

Dissertotion Abstracts Interntional is arronged by brood, generol subject cotegories. Flease select the one subject which most nearty describes the content of your dissertotion. Enter the corresponding four-digit code in the spaces provided.

Cumputer Science

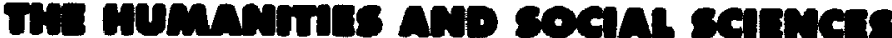

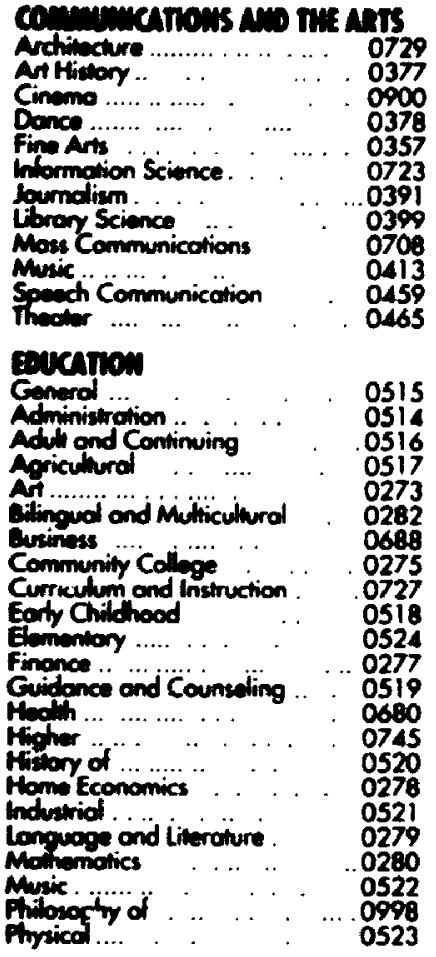

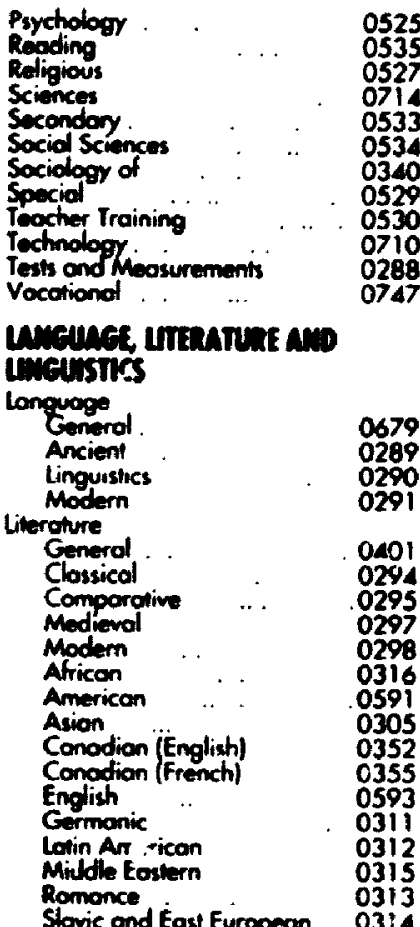

\section{mir semancts and Eneinatano}

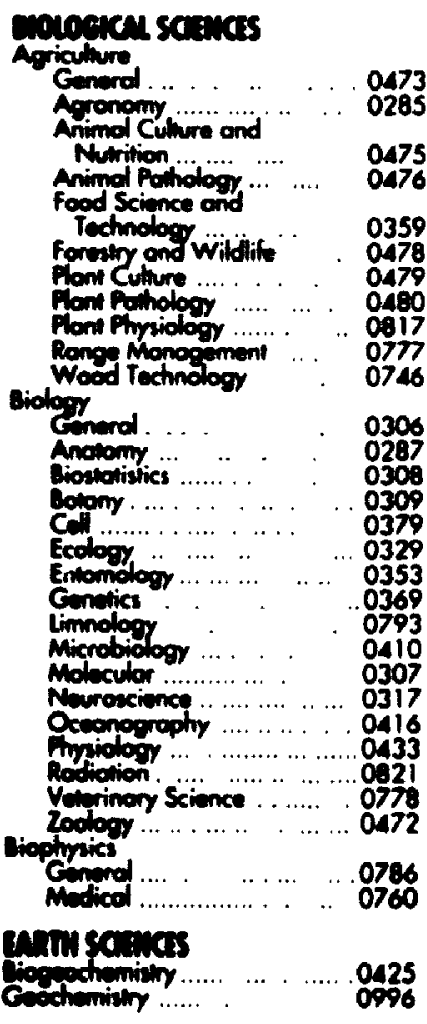

miosorir, nateren amp

inzelogr

Philosophy

Religion

Biblical Studies

Clergy

History of

Philosophy of

Theology

socell sognces

American Studies

Anthropology

Archooglogy

Physical

Business Administration

Generd

Accounting

Bonting

Monogement

Marketing

Conudion Studres

Economics

Generol
Agricuthurat

Commerce-Business

Finonce

History

Lobor

Folltore

Geogrophy

Geronlology

Mistory

General

0422

0318

0321

0319

0320

0322
0460

0323

0324

0326
0327

0327

0310

0272

0454

0338

0385

0501

0503

0505

050

0509

0510

0511

0358

0351

0578

suect $\cos$

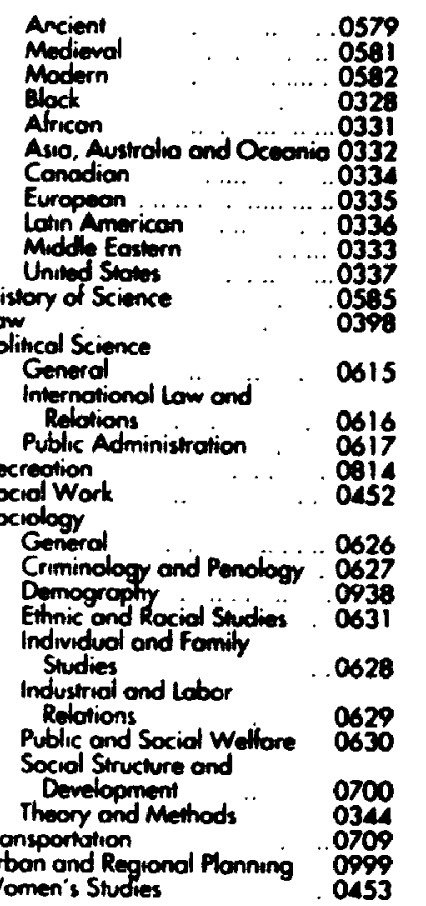

Speech Pothology
Toxicology

Horne Economics

mureal satucts

Pure Sciences

Chermisiny

General

Analytical

Broctiemisiny

Inorganic

Nucleor

tharmoceutical

Physicol

Polymer

Radiotion

Mathernotics

Phrsics

Generol

Acoustics

Astronomy and

Astrophynes

Amosphertc Scrence

Alomic

Electronics and Eloctricin

Elementory Porticles ond

High Energy

Fhud and Plosmo

Moloculo.

Nucloon

Optics

Sclit Stan.

038

Ophnhomolosy

Prormocology.

Phormocos

Prucel theropy

Rodic Hoont

Recrostion
Stotistics

057

Avind Scinnes:

Aplied Mechonics

Compuner Scinge
0460

0383

048

0749

0486

0487

0488

0490

0491

049

0495

0754
0405

0605

0986

0606

0608

0748

0607

0798

0759

0609

010

0752

0756

0463

0346

090
Engineering

Aenerol

Agrospoce

Automotive

Bromedical

Chemrcal

Civit

Electronics and Electricol

Heot ond Thermodynomic:

Hydroulic

industrica

Moleriols Science

Mechonical

Metallurgy

Mining

Poctoring

Petrobum

Sonivory ond Minicipol.......

Syrbem Science

Geowechnology

Operations Reworch

Plostics Technology

Textile Technology

0537

0538

0540

OS4)

0542

0543

0348

Prapleor

Generd

entovioral

Clinicel ....

Demolopinomiol

Experimontol.

industriol

Arronglion.

Pryiobopesel.

Puxchomentic:

Sociel

28

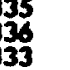

300

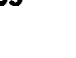


The undersigned recommend to the Faculty of Graduate Studies and Research acceptance of this thesis.

Integrating Process Modeling and Metrics Support

for Object Oriented Software Development

submitted by

Craig Robert Hayashi, BCS

in partial fulfillment of the requirements for the degree of Master of Computer Science
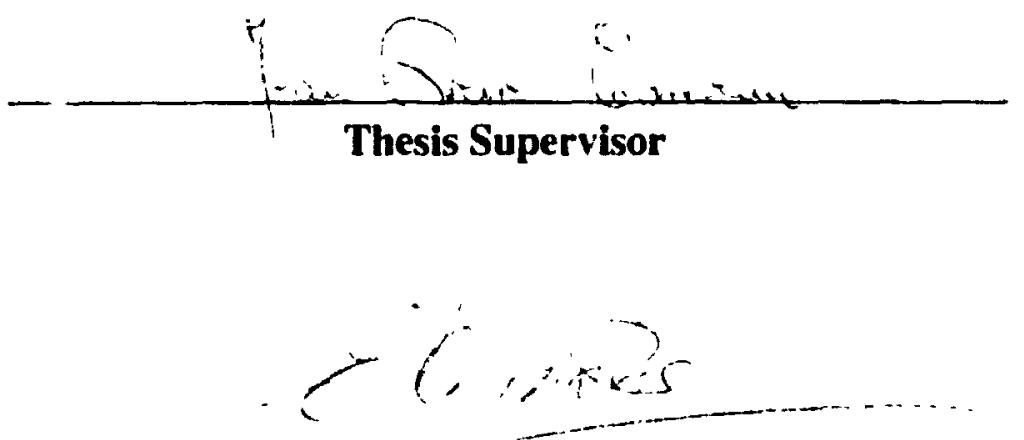

Director, School of Computer Science

Carleton University

November 10, 1994 


\section{Abstract}

Object-oric ited software development has come forth in recent years as a promising solution to the software crisis, which constitutes a crippling factor in our ability to realize large complex computer systerns. But object-oriented software engineering is still in its infancy and several of its facets remain largely ignored in the literature. In particular, little has been done in the domain of object-oriented project management.

In this thesis, a framework to assist with the project management and development of object-oriented systems is presented. The central idea of this framework is the use of process models to help with project management. The proposed approach offers several original and promising characteristics.

First, this framework is integrated with the development environment (namely, Smalitalk). Consequently, process models can be specified and scrutinized from a single environment. By directly consulting such models, developers can readily obtain guidance on how to follow the development process as they are proceeding with the development effort. Information on the progress of the project can also be automatically gathered from the development environment and supplied to managers. Furthermore, such an integrated approach has the virtue of avoiding the problem of inter-tool communication, which often plagues CASE (Computer-Aided Software Engineering) strategies.

Second, the use of process models allows a complex iterative process to be expressed as a sequence of tasks within each iteration. In other words, the spiral model generally associated with object-oriented development can be 'unwound' into a sequence of short iterations, each obeying the same process model. Consequently, the risk associated with an iterative process can be greatly reduced by carefully monitoring each iteration using a process model for it.

Finally, the proposed framework allows for the specification of metrics and their integration within the process models. For example, a userdeficed metric can act as the exit criterion of a task in a process model. Because the framework exists within the development environment, such metrics can be automatically and consistently collected. Thus, managers and developers can quickly analyze metric results and evaluate their usefulness in guiding further development. In other words, the framework facilitates the investigation of new metrics for object-oriented development. This is highly desirable given that a set of well-established metrics for object-oriented development still does not exist.

A tool named Vision was built to realize this framework. Vision provides significant benefits in project tracking, process reuse, process communication, metric collection, and metric analysis. 


\section{Acknowledgments}

I would first like to thank my advisor Jean-Pierre Corriveau. His knowledge, insight, and pragmatism were greatly appreciated throughout the duration of this thesis. I would also like to thank Newbridge Networks Corporation for providing me the opportunity to do this thesis. Thanks to Raluca Dragnea and everyone else at Newbridge who gave feedback and suggestions. Finally, thank you to Shehnaz Mohamed for always being there for me. 


\section{Trademarks}

ENVY/Developer is a registered trademark of Object Technology International Inc.

VisualWorks is a registered trademark of ParcPlace Systems Inc.

Other products may be trademarks of their respective companies. 


\section{Table of Contents}

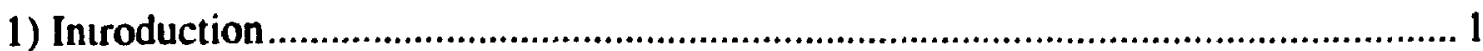

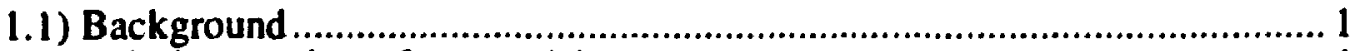

1.2) Solutions to the software crisis .............................................................

1.2.1) High level languages............................................................... 2

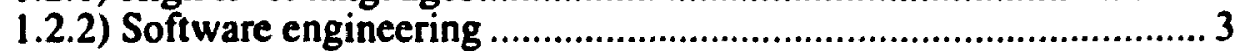

1.2.3) Computer aided software engineering ...................................... 4

1.3) Motivation .................................................................................................... 4

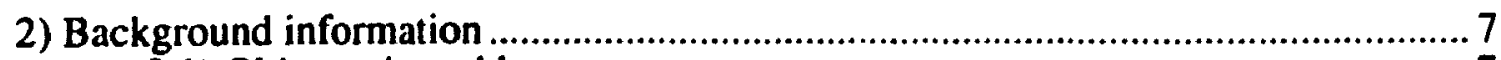

2.1) Object oriented languages ...................................................................

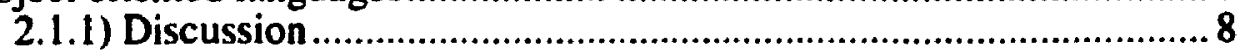

2.2) Object oriented methodologies .................................................................

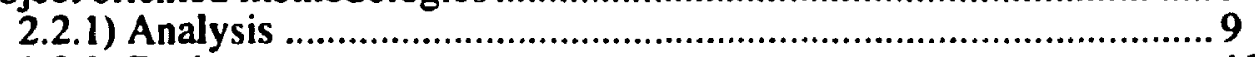

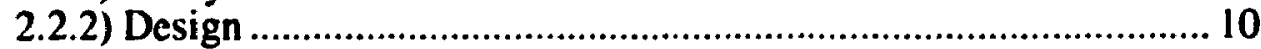

2.2.3) Implementation ...................................................................... 10

2.2.4) Quality assurance ...................................................................... 11

2.2.5) Maintenance ........................................................................ 11

2.2.6) How they are performed .......................................................... 12

2.2.7) Discussion .......................................................................... 13

2.3) Object oriented CASE tools........................................................................ 13

2.3.1) Discussion ................................................................................ 14

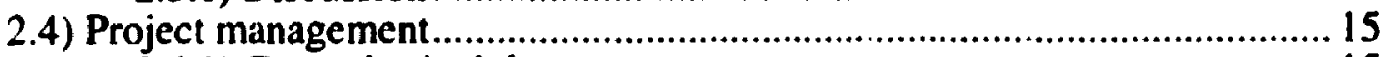

2.4.1) General principles .................................................................. 15

2.4.1.1) Planning .................................................................... 15

2.4.1.2) Tracking ........................................................................ 16

2.4.2) Object oriented project management ........................................... 17

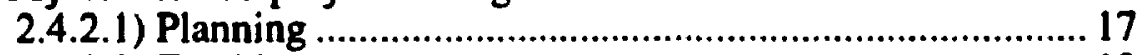

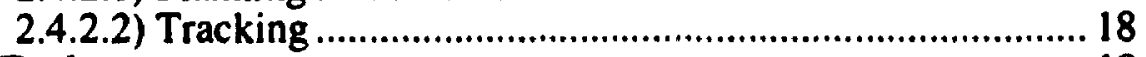

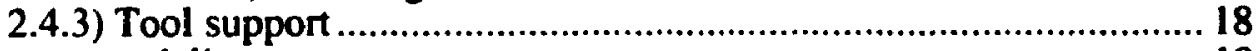

2.5) Process modeling .................................................................................. 19

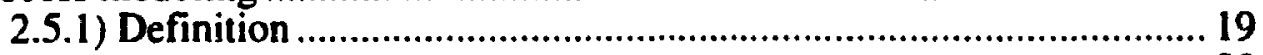

2.5.2) Uses...................................................................................... 20

2.5.2.1) Process communication ................................................. 20

2.5.2.2) Process improvement .................................................. 21

2.5.2.3) Project management ........................................................ 21

2.5.2.4) Process guidance and automation .................................. 21

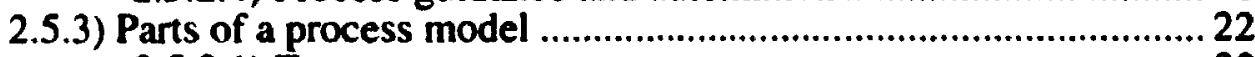

2.5.3.1) Types......................................................................... 22

2.5.3.2) Customization ................................................................... 26

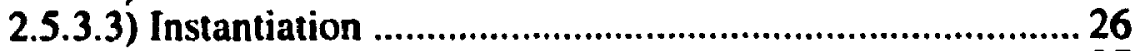

2.5.3.4) Enactment .................................................................. 27

2.5.4) Discussion ..................................................................................... 28

2.6) Metrics ................................................................................................ 29

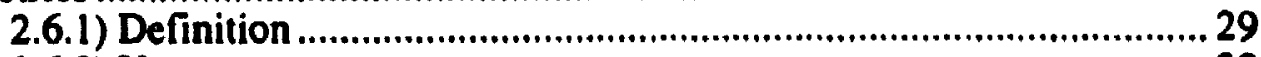

2.6.2) Uses............................................................................................. 29

2.6.3) Costs ...................................................................................... 31

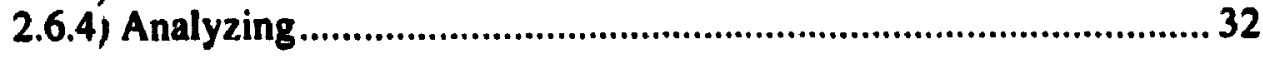


2.6.4.1) Mathematical tools........................................................ 32

2.6.4.2) Graph based tools ...................................................... 34

2.6.5) User's perspectives ...................................................................... 38

2.6.6) Ensuring acceptance ............................................................... 39

2.6.7) Object oriented metrics ............................................................... 42

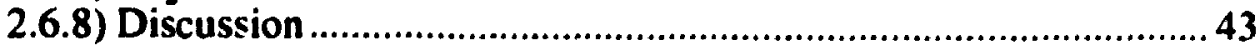

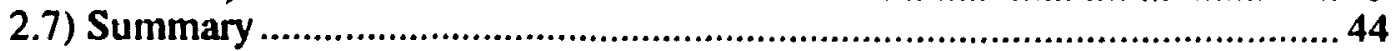

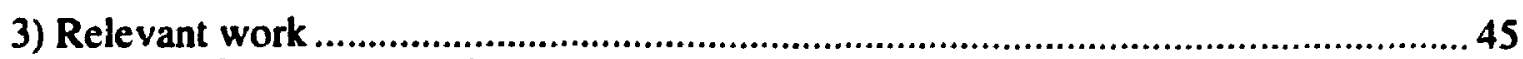

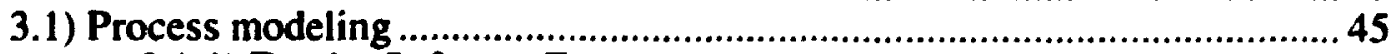

3.1.1) Eureka Software Factory ……….................................................45

3.1.2) Project Master Database .............................................................46

3.1.3) Visual Process Language ..............................................................47

3.1.4) Process Weaver ......................................................................... 48

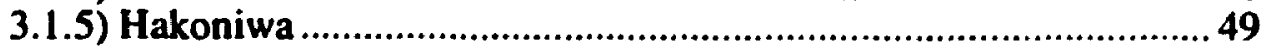

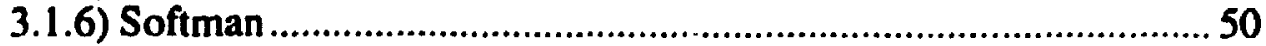

3.1.7) Amadeus ................................................................................... 51

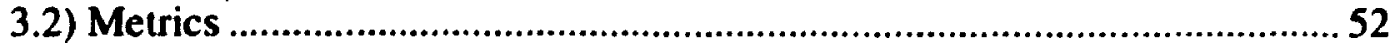

3.2.1) Productivity ................................................................................... 52

3.2.1) Quality ...................................................................................5 53

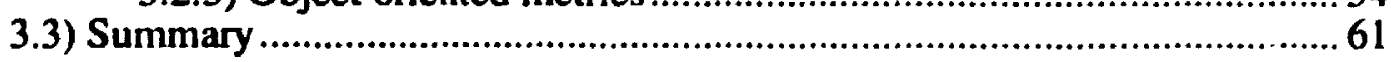

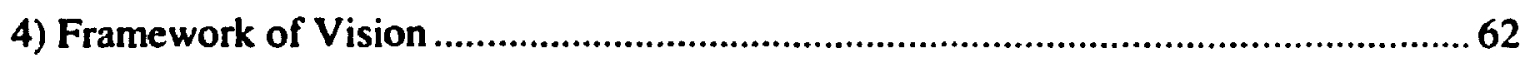

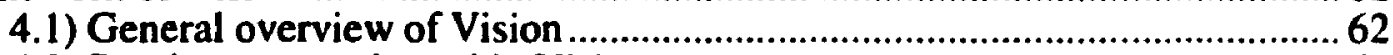

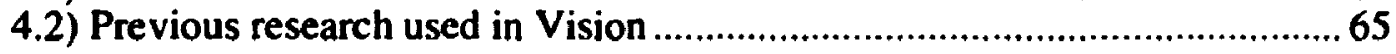

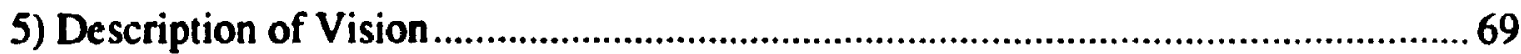

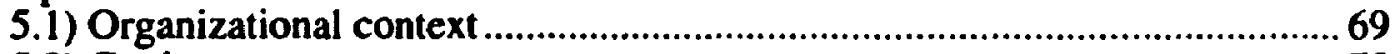

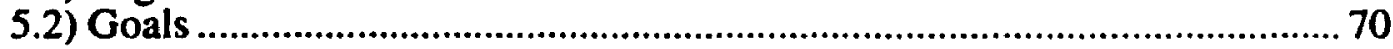

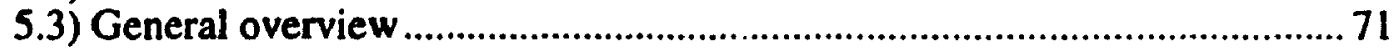

5.4) Process modeling ......................................................................................... 74

5.4.1) Process editor ............................................................................74

5.4.1.1) Process and phase levels ...........................................74

5.4.1 2) Process level objects ...................................................... 76

5.4.1.3) Phase level objects ......................................................... 80

5.4.1.4) Process editor features ..................................................83

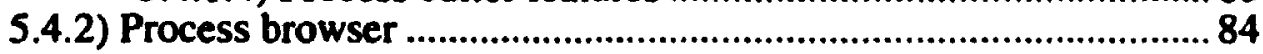

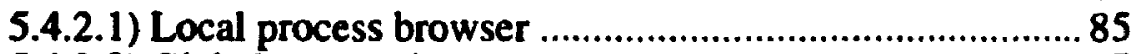

5.5) Metrics

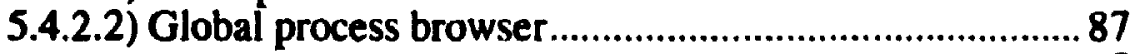

5.5.1) GQMA objects

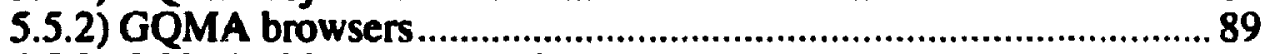

5.5.3) GQMA objects proposed ………............................................... 91

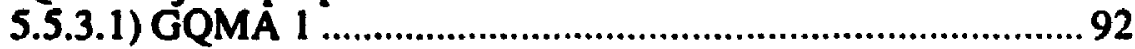

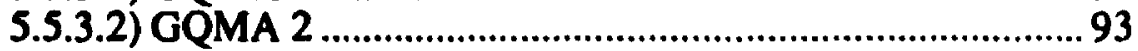

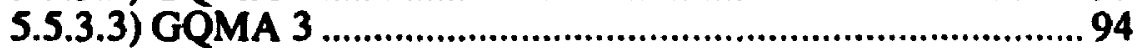

5.5.3.4) GQMA 4 ................................................................. 94

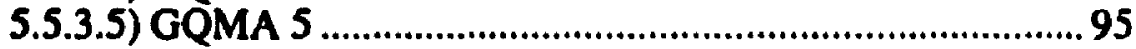

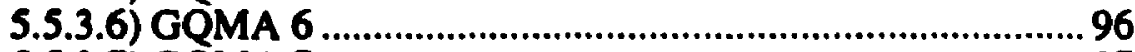

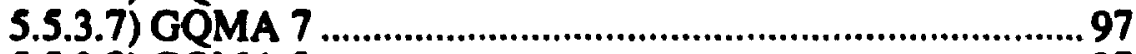

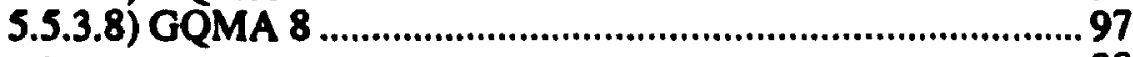

5.6) Management 


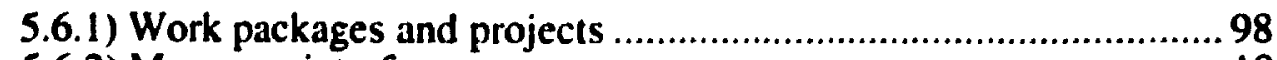

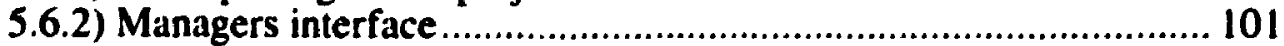

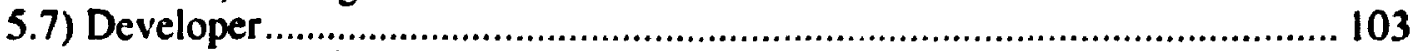

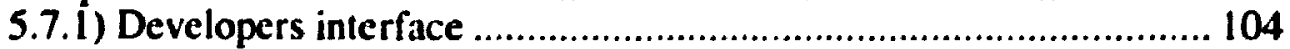

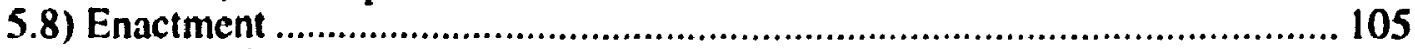

5.8.1) Process model viewer ....................................................... 106

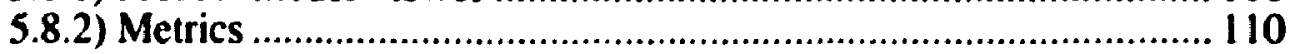

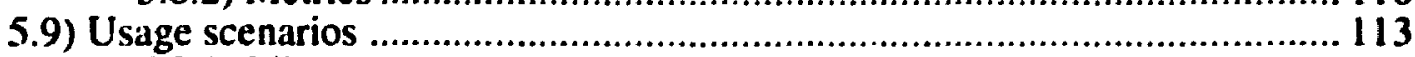

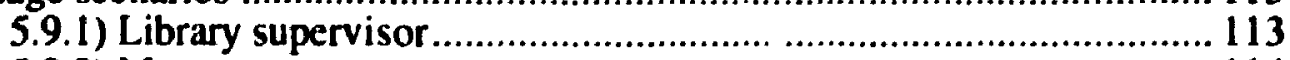

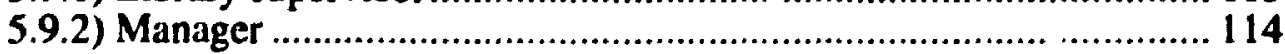

5.9.3) Developer .................................................................... 115

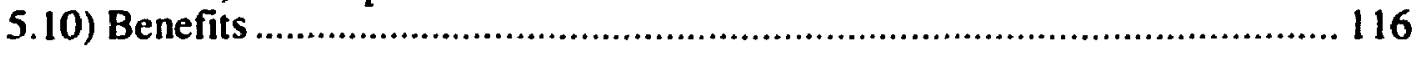

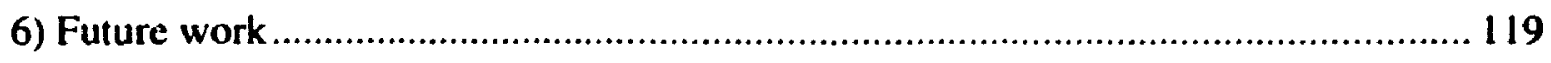

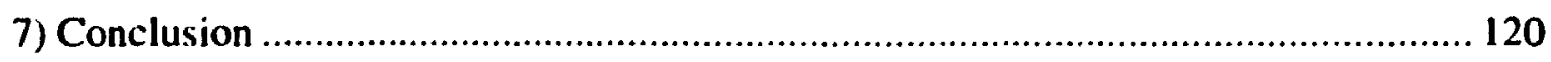

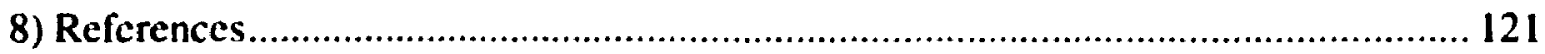

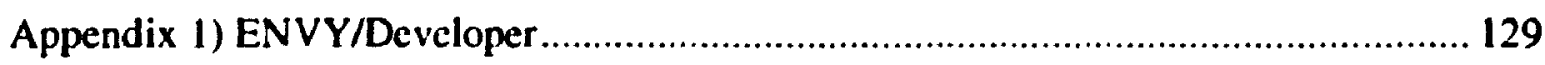

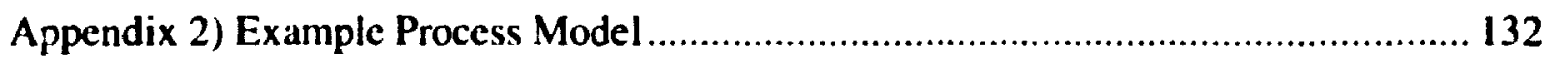

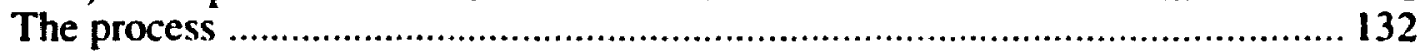

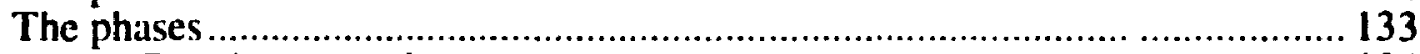

Requirements phase ................................................................. 134

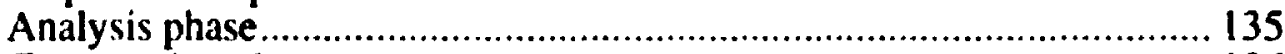

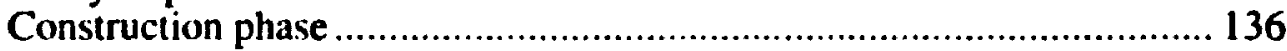

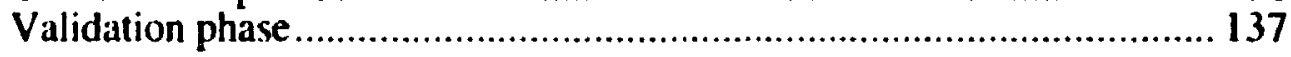

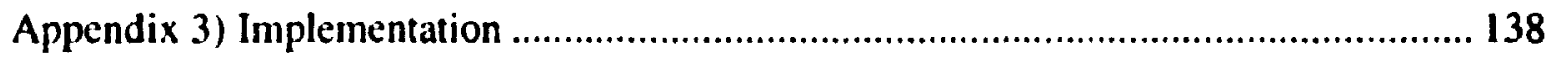




\section{1) Introduction}

\section{1) Background}

Since the beginning, hardware technology has advanced at a fast, but predictable. rate. Unfortunately, the ability to develop software has not kept pace with the hardware advances. In the early seventies, it was recognized that there was a software crisis [Sommerville, 1992]. This means that software systems were often being delivered late, over budget, and with a high number of errors [deMarco, 82]. Furthermore, maintaining these large software systems is now becoming increasingly more difficult [Lientz and Swanson, 1981].

As a result, developing the software is often the bottleneck in the ability to realize large complex systems. Therefore, lessening the software crisis by making it possible to develop high quality software in a reasonable amount of time and money will significantly advance the field of computer science. This provides the motivation for this dissertation.

\section{2) Solutions to the software crisis}

Several fields in computer science each provide important tools and principles to help with software development. These fields are high level languages, software engineering, and computer aided software engineering (CASE). A brief overview of each field is given below. 


\subsection{1) High level languages}

Computers are ultimately low level devices that work on sequences of bits. The first languages were very close to the hardware level. Programs were specified in terms of simple instructions. The best example of this type of language is assembler. It is considered a low level language because of its dependence on the hardware. Due to its lack of high level constructs, assembler programs are very tedious and time consuming to write.

Second generation languages provided a set of higher level constructs. These constructs better matched the programmer's representation of the problem. It was therefore possible to implement more complex prograuns. Examples of second generation languages are COBOL or FORTRAN.

Third generation languages (also called structured languages) further refined second generation languages by adding procedures. This made the control flow of programs easier to manage and allowed larger programs to be developed and maintained. Pascal and $\mathrm{C}$ are examples of third generation languages.

The latest generation of languages are called object oriented languages' ${ }^{1}$. These languages provide a new way of representing programs. They concentrate on the interactions of objects as opposed to the data driven approach of the previous generations of languages [Sommerville, 1992]. It is felt that objects are a more natural way for humans to think about a problem [Rosson and Alpert, 1990]. Two examples of object oriented languages are Smalltalk and C+t.

\footnotetext{
'Note that 4th generation languages are not covered since they refer mainly to husiness oriented applications as opposed to general programming languages.
} 


\section{Commentary}

Higher level languages have helped to close the gap between the way that humans and computers represent the problem domain. This increases the productivity of programmers and allows larger and more complex applications to be developed. The languages also scek to minimize the number of errors that can be introduced into the code. High level languages, therefore, help ease the software crisis by attempting to provide the best possible way to implement a solution on a computer.

\subsection{2) Software engineering}

As larger and larger applications were developed, it was recognized that the manner in which software was developed was just as important as developing the software. Software engineering seeks to make the development of software a science as opposed to an art [Sommerville, 1992]. To this avail, it calls for the use of a development process to specify how software is to be developed and maintained. The process defines the specific order of tasks and quality controls to be followed. Metrics are used to track the development process and development product - namely the code.

\section{Commentary}

The benefits of having a formal methodology to develop software is that it becomes possible to measure and control the development. Management tools can be used to ensure the development is going according to schedule. Well-defined deliverables increase the quality of the product. Furthermore, by measuring the activities and products of the development process, it becomes possible to analyze and improve it. 
Software engineering principles help lessen the software crisis by working to improve the manner in which software is developed.

\subsection{3) Computer aided software engineering}

Computer aided software engineering (CASE) is a broad term used to encompass software tools to help with the development of software. Examples of CASE tools are programming environments, configuration management tools, debuggers, or high level analysis and design tools [Sommerville, 1992]. The goal of CASE tools is to use the power of computers to help automate and assist certain aspects of software development.

\section{Commentary}

When the idea of CASE was first proposed, there were great hopes and expectations as to what benefits it would provide. However, several problems, such as inter-tool communication and integration, have plagued the CASE enterprise [Wilkie, 1993, p.162]. Nevertheless, CASE tools do provide important and necessary support for the development process. There is still great potential that CASE tools can significantly contribute to easing the software crisis.

\section{3) Motivation}

As it was previously mentioned, the overall goal of this research is to help improve the manner in which software systems are developed. The research encompasses the three fields that were discussed in the preceding section. 
Object oriented technology is now coming into its own. Numerous companies have developed software using the object oriented paradigm. Their success and validation of the benefits of object criented technology have inspired other companies to use it [Meyer and Nerson, 1993]. As a result, object oriented software development has a strong foothold in industry. The field is still maturing but the benefits are real. For these reasons, it is felt that there is much room for significant and beneficial research in this area.

Within the object oriented world, there are many languages and methodologies available. Out of these, there are a few strong contenders. However, due to the abundance of active research and products in these areas, proposing another object oriented language or methodology would be of limited benefit. Areas that are still little researched include project tracking and metrics [deChampeaux et al., 1993]. These areas have an important impact as to the success of developing software on time and of high quality. Furthermore, CASE support for object oriented project tracking and metrics has not been thoroughly explored.

This thesis concentrates on proposing a framework to help with development and management of object oriented projects. The central idea of this framework is the use of process models to help with project management. The proposed approach offers several original and promising characteristics:

1) the framework is integrated with the development environment

2) the use of process models simplifies the management of an iterative process

3) the framework allows for the specification of metrics and their integration within the process models 
The advantages of these characteristics will be discussed at length throighout this dissertation. For now, it is important to understand that the intent of this work is not to motivate a particular management strategy or set of 'correct' metrics for object-oriented development, but instead to focus on the expression of such artifacts 'on top of an existing development environment, namely VisualWorks. The process models and metrics presented here are therefore merely illustrative. Indeed, at this point in time, it appears that the notions of a coirect project management strategy and of a correct set of metrics are quite problematic [Jacobson et al., 1992; Meyer and Nerson, 1993] for, in object-oriented software engineering, each problem typically requires a specific solution, only constrained rather than determined by general guidelines and strategies.

To demonstrate the framework's feasibility and validity, a tool was built to realize the framework. The tool is called Vision. The scope of this thesis is to motivate and describe the development of the framework and Vision. Specific uses and results of using Vision are not discussed here but rather left as an area of future research.

To ensure that Vision is as industrially relevant as possible, a research partnership was formed with a local company. The company's name is Newbridge Networks Corporation. Newbridge markets both hardware and software support for wide area data networks. At the time of this thesis, Newbridge was just beginning to adopt object oriented software development. Therefore, this provided an ideal opportunity to develop Vision to use in an actual object oriented software development project.

The remainder of this thesis is organized as follows. In the next two sections, the appropriate background information and relevant work will be presented. Vision will then be described and its benefits discussed. The final section will give conclusions and areas of future research. 


\section{2) Background information}

\section{1) Object oriented languages}

Object oriented languages can trace their beginnings to the language Simula ${ }^{2}$ [Meyer, 1988]. Simula was first used as a simulation language and therefore modeled the real world interactions of objects. The concept of an object was applied to general purpose languages in later versions of Simula and subsequent object oriented languages that were developed [Budd, 1991].

With object oriented languages, the basic building block is the object. Objects are defined to have state (private data) and behaviour (functions) [Booch, 1991]. Software systems are viewed as a collection of interacting objects. This is in contrast to the previous paradigm where systems were composed of data and functions that operated on the data.

Four defining characteristics of object orientation are messaging, encapsulation, inheritance, and polymorphism [Henry and Humphrey, 1990]. Messaging refers to the fact that objects communicate with each other by sending messages. Objects are therefore passive entities that respond to messages that they are sent. Encapsulation means the object's state can only be accessed through the object's public message interface. Inheritance allows child objects to share common state and behaviour with a parent object. Finally, polymorphism permits different objects to handle the same message in different ways.

${ }^{2}$ The first versions of Simula were object based but later versions were object oriented. 
As a result of these features, the object oriented paradigm is viewed as a better way to develop software [Wirfs-Brock et al., 1990]. Some of the advantages are as follows. Since objects are concrete entities that have real world counterparts, objects better match the human and computer representation of the problem [Rosson and Alpert, 1990]. Objects are also more reusable which increases the reliability of systems and the productivity of developers. Finally, it is easier to fix bugs and add functionality to object oriented programs [Booch, 1994].

There are many different research and commercial object oriented languages available. In industry, the two most popular object oriented languages are Smalltalk and $\mathrm{C}++$. Each of the languages comes with a programming environment where development is done. This environment contains browsers, editors, debuggers, and graphical user interface builders [Goldberg and Robson, 1983].

\subsection{1) Discussion}

In industry, object oriented languages have proven themselves and are used in real world systems [Meyer and Nerson, 1993]. As it was discussed in the previous section, the notion of a development environment offers a powerful and integrated set of tools to help develop code. This seamless integration of tools provides the developer with convenience and productivity gains.

In keeping with this model, Vision is completely integrated with the development environment. This will evolve the development environment to contain project tracking and metric specification tools. With Vision, the environment gives support for both the development process and the development product (the code). 


\section{2) Object oriented methodologies}

While object oriented languages provide the medium to develop software, object oriented methodologies provide the steps to follow in order to develop software. In following with software engineering principles, development is defined as a series of phases. A typical development lifecycle contains analysis, design, implementation, quality assurance, and maintenance phases.

\subsection{1) Analysis}

In the analysis phase, the real world problem domain is studied. The goal of analysis is to produce a description of what the system must do. The system is described in terms of the problem environment without considering computer related isslies. It should capture the requirements the automated system must fulfill, knowledge learned about the application domain, and how the system will interact with the problem domain [Høydalsvik and Sindre, 1993].

The results of object oriented analysis include a description of the objects in the problem; identification of classes and their attributes, behaviour, and relationships; and documentation of the manner in which the objects interact with each other [Monarchi and Puhr, 1992]. The end result after analysis is a thorough understanding of what the software system must do and how it fits in with the real world problem space. The documents that are created are used for two purposes. First, they serve as a communication medium between analysts and customers to ensure the customer and developer agree on what the system will do. Secondly, they form a bridge from analysis to design by telling designers what functionality the system must have. 
2.2.2) Design

From the system described in analysis, a solution to realize the system is produced in design. This forms the solution domain and tells how the system will work. This includes a detailed description of the system's parts and how they interact with each other [Høydalsvik and Sindre, 1993]. It does not go into coding details and is therefore language independent.

The artifacts that are produced in design are similar to those produced in analysis. Classes and the interaction between objects are again described. The design will include objects that are not explicitly in the real world problem domain but are required in order to realize the software system. Notwithstanding, many of the objects identified in analysis form part of the design solution.

How detailed design goes can vary from project to project. Nevertheless, any design effort will focus on refining the objects so they are encapsulated, form meaningful hierarchies, and are reusable. From the design, the implementation of the system begins.

\subsection{3) Implementation}

Implementaiion concerns the language specific code to implement the system on a particular hardware platform. Implementation is the final step towards getting the system to run. The class hierarchies, class descriptions, and method definitions will be compiled to produce the machine executable instructions that fulfill the system's requirements. 
The level of detail described in the design phase will determine how closely the design and implementation match. At any rate, implementation should augment the design with language or hardware specific constructs that optimize the system's performance and implementation.

\subsection{4) Quality assurance}

Quality assurance activities take place in all three of the previously discussed phases. Quality assurance is done to ensure that each phase correctly accomplishes its goals. Quality assurance in analysis determines that the described system correctly models the real situation and requirements it must meet. Quality assurance in design checks that the system correctly fulfills the requirements. Quality assurance in implementation makes sure the code properly implements the design. Additionally, quality assurance in each phase is performed to ensure that the solution will work. In implementation, this means the program will run without causing any errors. At the analysis and design levels, this quality assurance can take the form of logical walkthroughs or simulation.

\subsection{5) Maintenance}

In order to develop and deliver the system, the four phases of analysis, design, implementation, and quality assurance are performed. Any activities after the delivery are termed maintenance. This can include bug fixes in the system, evolving the system due to changes in the problem domain, or extending the system to give more functionality [Hall, 1992]. 
Maintenance will encompass the tasks of analysis, design, implementation, and quality assurance. The added difference is that the existing system must be first understood in order for it to be modified [Chapin, 1988].

\subsection{6) How they are performed}

Object oriented methodologies were not the first to introduce the concept of a phased development effort. The structured paradigm also uses a phased development effort with the same phases as described above. The difference with object oriented methodologies is how the phases are performed.

In the structured paradigm, a phase must be completely finished before the next phase is started. This is called the waterfall model of software development. The object oriented paradigm, follows an iterative or spiral model [Boehm, 1986]. Under this model, a small fraction of each phase is completed. This constitutes one cycle or iteration through the lifecycle phases. The end result is a coded and tested product that implements a small part of the overall system. These cycles are continuously performed until the entire system is realized. Therefore, the development of the system can be viewed as a spiral that consists of many successive cycles through the lifecycle phases.

There are several advantages to the incremental development process that the spiral model supports. One of the main benefits is that information learned during a cycle can be applied to subsequent cycles. This allows the design to evolve towards the final product. It also provides incremental releases of the product during the development effort. This is useful to show progress and to ensure the system is developed as the customer expects. Since the system is incrementally built up, there is no big integration 
phase at the end. Furthermore, testing is distributed across the cycles so errors are detected earlier.

\subsection{7) Discussion}

Although the same phases are performed in each cycle, the cycles need not be identical throughout the entire development. For example, the initial cycles may spend more time in the requirements phase because so little is known about the problem. As more of the solution is implemented in the middle cycles, the majority of time may be spent in the design and implementation phases. Finally, the later cycles may spen:? more time integrating and testing the new code with the old code.

None of the current methodologies go into any great detail about the dynamics that occur during the spiral process. Capturing data such as this will lead to a better understanding of the object oriented software development process. This research is dedicated towards the development of a tool to track a methodology. In the future, after Vision is completed and used to track several projects, it should provide accurate information as to how the development process was performed. This data can be used to further research of object oriented methodologies.

\section{3) Object oriented CASE tools}

There are many object oriented CASE tools available. They can be grouped into two general (possibly overlapping) categories - development centered and methodology 
centered ${ }^{3}$. Development centered tools focus on assisting with the development of code. Examples of this kind of tool are debuggers, graphical user interface builders, or configuration management tools. Since they mainly help with implementation, they are closely integrated with the language and programming environment.

Methodology centered tools give support for a particular object oriented methodology. The tools provide features to help the user work with the notation that a given methodology uses. The system can be graphically described and code stubs can be generated. By supporting the development of a system using graphical notations, development is raised to a higher level than coding. The tools strive to be analysis and design tools so they are language independent. There is no close integration between these tools and the programming environment.

\subsection{1) Discussion}

Within the object oriented CASE tool world, there is an important gap between the development centered and methodology centered tools. There are no tools to support the process that takes place during the development of the system. Pruject tracking and metric collection do not appear to be handled by any of the tools. The development centered tools are too low level and the methodology centered tools are too high level. This research addresses this gap and the proposed tool provides important and necessary computer support for these tasks. This gap is significant because tracking and metrics allow measurement and measurement allows a project to be controlled [deMarco, 1982].

\footnotetext{
${ }^{3}$ Note that the catcgory names were chosen because the development centered tools are primarily used to support development and the methodology centered tools are primarily used to support a methodology. The names are meant to best characterize how the tools are used, nothing more. In other words, it is quite possible that a tool may apparently belong to both categories, in which case its main role should determine the category to which it does belong.
} 


\section{4) Project management}

\subsection{1) General principles}

As the name suggests, project management is a term that describes the tools and techniques that are used to oversee the completion of a project [Zells, 1990]. It covers all aspects of the project from planning the tasks, scheduling resources to do the tasks, and tracking the progress of the tasks that make up the project. Project management is a general field and is applicable to all types of projects, not just software development projects.

\subsubsection{1) Planning}

The first step in managing a project is to identify the jobs that have to be performed in order to complete the project. Each job or task contains a description of the job, the tools and skills needed to do the job, inpui and output deliverables, and criteria for completion. Next, the effort that each job wil! equire is estimated.

From this information, the jobs can be scheduled and a project plan can be devised. The project plan onders the jobs to account for any dependencies among the tasks. The resources are then assigned to the tasks, taking into account availability constraints. The project plan should be constructed as to allow as much parallelism as possible between the jobs and make the best use of the resources.

The project plan is usually represented graphically. The most popular method of doing this is by using a PERT chart [Reuter, 1979]. On a PERT chart, boxes or nodes are 
used to represent a job. The start date, completion date, resource assigned, and percent complete for a job is printed in its box. Edges connecting the nodes capture the dependencies among the jobs. By placing and connecting all jobs of the project in the PERT chart, a network is created. From this network, the critical path can be calculated. The critical path gives the minimum time required to perform the jobs as they are ordered in the PERT chart. Alternate PERT networks can be tried to see if they better utilize the resources and give a shorter critical path.

\subsubsection{2) Tracking}

During the span of the project, time, costs, and resources can be tracked. This will ensure the project is proceeding according to how it was planned. Any deviations from the plan will mean the schedule, budget, or resources required will have to be adjusted.

Time is tracked by regularly logging the time spent on each job. From this, the total time spent on a job can be calculated. When this is paired with an estimate of how much more time is required to complete the job, the probability of finishing the job by the scheduled completion date can be determined. Using this information, corrective action can be undertaken. For example, if a job has used 80 percent of its allotted time but is only 50 percent finished, there is a high probability that it will not be finished on time. If the job is on the critical path, a slippage in its time of completion will affect the length of the entire project. It may be necessary to divert additional resources to the job so that it is completed in the scheduled time.

In a sinilar fashion, the costs incurred and resources used are actively monitored against their target values. The key to project success is to ensure that the project is 
completed according to its plan. The only way that corrective action can be performed is if potential problem areas are be discovered and monitored.

\subsection{2) Object oriented project manageisent}

The project management techniques that were described above are fairly general and could be applied to any type of project. When an object oriented software development effort is managed, there are some specific issues that have to be addressed [Pittman, 1993].

\subsubsection{1) Planning}

As it was discussed in the section on methodologies, object oriented software development is performed according to an iterative model. This means the project evolves as it is developed and change is a fundamental part of the process. It is not possible to give a detailed plan of the entire project from start to finish because what is done in the later stages of the project depends on the work that is done in the initial iterations. However, a general idea of what will be done and when it will be done by is calculated by estimates. For example, the system's requirements will become clearer as the project proceeds. Therefore, at the beginning of the project it is not possible to tell what features will be developed near the end of the project because those features have not been discovered yet.

Although the precise nature of future tasks is unknown, the process specifying how they are to be developed is known. At the beginning of the project, the number and duration of the lifecycle itc.atinns that will be done are planned out. An iteration will consist of the various phases as given by the methodology. These phases are further 
broken down into the steps required to complete each phase. Even though the content (work to be done) of each iteration is not known in advance, the structure (process to follow) of each iteration is well defined and repeats over the entire project.

\subsubsection{2) Tracking}

With an object oriented project being prone to change, it places greater importance on tracking. The project must be well tracked so that any changes are detected and absorbed into the plan. The best way to accurately track a project is to have tasks that do not take a long time to complete (e.g. 3 to 7 days of work) [Pittman, 1993]. The tasks are defined to be 0 or 100 percent complete. This eliminates the 90 percent done / 90 percent remaining phenomenon that occurs because the last 10 percent of a job usually takes a disproportionate amount of its time [Pittman, 1993]. The downside of tracking such small work packages is the added overhead of breaking down work into the small tasks and tracking each of the tasks.

\subsection{3) Tool support}

For general project management duties, there are several powerful software packages that provide automated assistance. The features of these tools include graphical display of PERT charts, calculation of the critical path, assignment of resources based on calendar availability, and budget calculation [Zells, 1990].

What is lacking are tools to support the management of object oriented software

development projects. As it was discussed, these types of projects are characterized by a regular structure of iterations and a need for fine grained tracking. This research shows how process modeling can take advantage of an object oriented project's repetitive 
structure to provide detailed tracking without adding extra overhead to the developers or project managers.

\section{5) Process modeling}

\subsection{1) Definition}

A process is defined as a set of partially ordered steps that tell how to accomplish a goal [Shepperd, 1992]. The words 'process' and 'methodology' are often used interchangeably to describe how software is developed. Any software development effort will follow some sort of process. However, depending on the organization, the process will vary as to how detailed and complete its steps are. There are now recognized organizations that will certify a process as meeting certain minimum quality standards 4 . This is recognition that a well defined process with the appropriate quality checkpoints is a determining factor in the quality of the software that is produced.

A model is defined as '... an abstract representation of reality that excludes much of the world's infinite detail.' [Curtis et al., 1992]. In other words, models are used to reduce the complexity of understanding a phenomenon by eliminating the detail that does not influence its relevant behaviour. Models have long been used to experiment and predict situations that were not feasible or cost effective to do in real life. For example, models are used to predict weather or simulate how buildings react to earthquakes.

\footnotetext{
4 Such certification presupposes a well-accepted lis of evaluation criteria for the process under scrutiny. Such criteria have not yet been established for object-oriented development.
} 
Taken together in the context of software development, a process model is defined as a representation of how a software development process is performed in the context of the goals and constraints of a given organization [Krasner et al., 1992]. The model formally captures the steps, who does the steps, what resources are required, and in what order they are to be performed. A process model is a more detailed representation of a process because it shows how the process fits into an organization and how it interacts with its resources.

\subsection{2) Uses}

Process models have many uses to an organization [Curtis et al., 1992]. These uses are discussed in the following sections.

\subsubsection{1) Process communication}

One benefit of a having process model is that it facilitates human understanding of the software development process. In many organizations, the development process is not understood because it was never formally captured. Development proceeds according to some loose guidelines that are heavily supplemented by individual developers' experience. As a result, there is no clear and accurate way to determine how the development process works. This makes it harder for new developers to learn how to develop software and results in valuable information being lost when an experienced developer leaves.

By having a process model, the process is easy to understand because it is represented in a common and consistent format. Furthermore, it allows people to unambiguously exchange their ideas about the process. 


\subsubsection{2) Process improvement}

After the development process has been modeled and understood, it is possible to analyze it. The model can be examined for areas that $2 \mathrm{e}$ inefficient or prone to error. Modifications to the process can be proposed to address these areas. Most importantly, the improved mociel can be compared to the old model to ensure that the modifications were indeed beneficial. This supports the evolution and continual improvement of the process. The most effective processes can be reused across projects.

\subsubsection{3) Project management}

Since process models represent the development process, they provide a template to which actual project behaviours can be compared. This helps with project management because it is possible to track people's project related activities against the process model. Compared to a project plan, the level of tracking is much finer with the process model because the model gives the precise steps required in order to complete a task.

\subsubsection{4) Process guidance and automation}

While managers can use a process model to better track a project, developers can use a process model to help them follow the development process. By using a process model, the developers have a clear guide as to how to develop as specified by the process. The process model can tell the developer the appropriate template files or reference material to use at a given point in the process. 
If the process model is represented on-line in a CASE tool, there is the possibility of providing automated assistance to the developer. The tool can be kept up to date as to which step the developer is currently working on. Given this information, the tool can actively help the developer. For example, it can automatically start the correct tools and open the necessary files in order to do a task. This will increase the developer's productivity since the mundane tasks will be done by the computer.

\subsection{3) Parts of a process model}

In this section, the different parts of a process model will be discussed. First, the different types of process models will be presented. Then the steps on how to use a process model in a project will be given.

\subsubsection{1) Types}

Within a process, activities interact with resources to produce a product. Therefore, a process model must somehow represent all three of these entities. Different types of process models put different emphasis on each of the entities [Fernström, 1993]. Table 1 gives a summary of the types and how they each represent process information.

\begin{tabular}{|c|c|c|}
\hline Type & Boxes & Lines \\
\hline Product centered & Product states & Allowable state changes \\
\hline Activity Centered & Activities & Flow of products \\
\hline Resource centered & Resources & Flow of products \\
\hline
\end{tabular}

Table 1: Information represented by process models 
Product centered models (also referred to as deliverable centered models) concentrate on the product. The model shows the state information of the product as it proceeds through the process. Activities and resources are considered a means to change the state of a product. An example of a product centered model is shown in figure 1 .

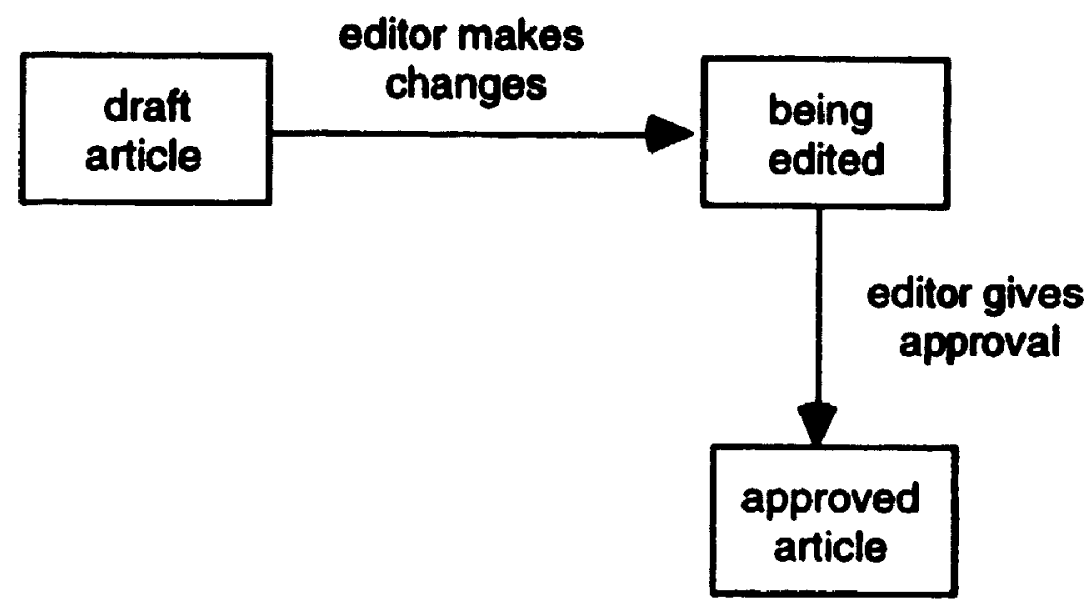

Figure 1: A product centered process model

The model depicts a very simple process where a document is edited and approved for release 5 . The boxes represent the various states of the product (the document) and the arrows specify the allowable sequence of state changes. As it can be seen from the figure, the model gives a clear indication of the various states the product can be in.

Activity centered models place their emphasis on the activities in the process. An example is shown in figure 2.

${ }^{5}$ Note that this is only a fictional example and is not meant to depict any actual process. In other words, it is irrelevant to the discussion at hand whether editing includes an approval sub-phase in itself or whether it is followed by a review state. 


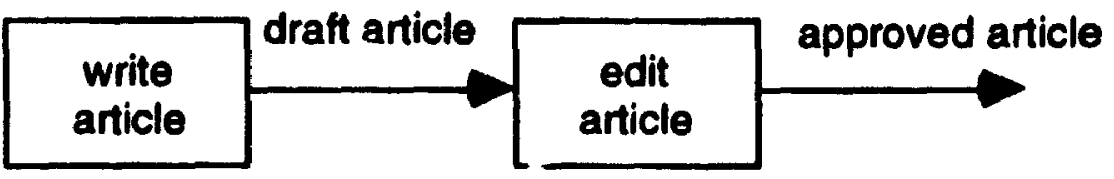

Figure 2: An activity centered process model

The same process used in figure 1 is now shown from an activity centered view. The boxes represent activities and the arrows show the flow of products between the activities.

The last type of process model is a resource centered model. A resource centered model of the editing process is shown in figure 3.

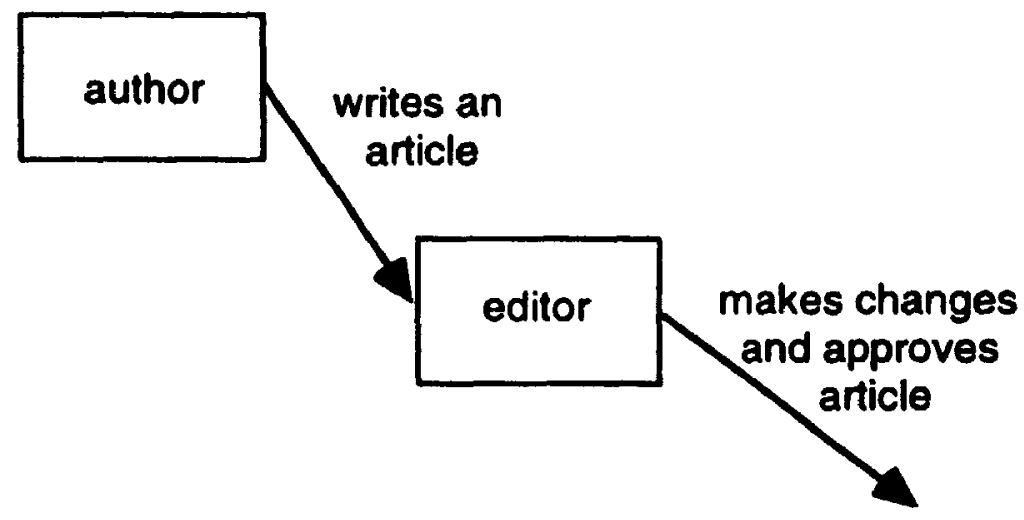

Figure 3: A resource centered process model

The boxes represent the resources that perform the activities in the process. The arrows are used to show the flcw of products between the resources. 
As it can be seen from the examples, each type of process model gives more importance to one of the entities involved in the process. The type of process model that should be used therefore depends on the objectives of the modeler. If it is desired to use a process model to guide people on how to follow a process, then an activity centered process model is the best choice. As an example, consider a new employee following a process model on how to get approval for a purchase order. The process model will show the employee the steps that are required and the order in which they must be done.

To track a product, a product centered process model should be used. A manager using a product centered model to track the development of an application is an example of this use. The model will allow the manager to determine what state the application is in (e.g. developed, tested, released) at any point in the development effort.

To manage resources, a resource centered model is the most appropriate. For example, modeling the construction of a house from a resource centered view will show when the various resources are active. This will allow scheduling of resources at the appropriate times (e.g. plumber, electrician, brick layer, ...).

The preceding description on the different types of process models has been general and abstract. Actual process models may not fall exactly into one of the three categories. In other words, they can be termed hybrid and contain multiple representations of information. The models will also differ as to how the information is expressed. In the section on relevant work, some example process modeling systems will be described. 


\subsubsection{2) Customization}

Once it has been determined which type of process model will be used, the model Inust now be customized to a specific project [Basili and Rombach, 1987]. This involves capturing the project's development process in the process model. The process model must capture the steps in the process with respect to the project's goals. For example, if the project involved developing a system to control a nuclear power plant, the process model should include stringent quality assurance steps. This is because testing and reliability of the software system are important goals. If the project involved adding new features to an existing application to keep pace with a competitor's product, the model may lessen testing steps to quicken development. This would match the project's goal of speed of development.

The power of using computerized process modeling tools is that previous models are stored and are readily retrievable. This allows an organization to build up a library of process models to match the needs of any given project. The need to create or customize process models to a specific project decreases as more and more process models are saved in the library.

\subsubsection{3) Instantiation}

At the end of the customization stage, a process model that is suited to a specific project is available. This can be thought of as a master template for the project. In order to use the process model in the project, it has to be instantiated. This involves creating copies of the process model and setting its parameters appropriately [Notkin, 1988]. This will allow the process model to accurately be used in the project. 
For example, each developer in the project will be given a separate copy of the process model. Each copy will specify the documents and code that will be created by the developer. They will also specify the schedule the developer must adhere to. When the appropriate process model is given to a developer, he or she has an accurate of record of what they must do over the life of the project.

\subsubsection{4) Enactment}

The final step to using a process model in a project is enactment. This means the process models are followed and the process is carried out as it is described in the model. Enactment can take different forms depending on the degree of coupling between the process model and the environment [Fernström, 1993].

In an active support system, the process modeling system is tightly integrated with the project environment. The modeling tool has access to the various tools and files that are used during development. The developers keep the process model synchronized as to what task they are currently working on. In response, the modeling tool will run the appropriate tool with the appropriate file for the current task. This relieves developers from some of the manual tasks of software development.

A process enforced system takes this one step further. It will control access to the tools and files. The only way in which the developers can access the tools and files is if it is needed for the current task in the process. This forces developers to adhere to the process as specified by the model.

At the opposite end of the spectrum are loosely coupled systems. In these systems, there is no automatic connection between the process model and the project 
environment. The process model is primarily used to guide developers as to what to do next and how to perform a task.

This completes the discussion of process modeling. In the next section, the previous information on process modeling is related to the current research.

\subsection{4) Discussion}

Vision includes process modeling capabilities. With respect to process modeling, the two main goals of the tool will be to help managers track the project and to provide assistance for developers in following the process. Given these goals, Vision uses activity centered process models to represent the process. It is felt that activity centered models will provide the best balance between guiding developers and tracking the process. Resource or product centered process models place too much emphasis on their respective entities to be simultaneously useful to both managers and developers.

Vision also aims to provide as much active process support as possible. This is possible because Vision is completely integrated with the development environment. However, Vision does not enforce a process because it may stifle the productivity and creativity of developers.

In the section on relevant work, some existing process modeling tools will be surveyed. These tools were all developed for use with projects using structured languages. This thesis represents the first known process modeling tool to be developed specifically for object oriented software development. With the tool being integrated with the Smalltalk development environment, it has access to the code produced during development. This makes automated metric collection possible. As it will be shown in 
the next section, combining process modeling with metrics collection is the source of the real power of Vision.

\section{6) Metrics}

\subsection{1) Definition}

Metrics are a way to characterize some aspect of a product or process by assigning them a numerical value [Fenton, 1991]. Product metrics measure features of the work produced. For example, lines of code is a product metric on the code produced as a result of a software development effort. Process metrics measure features of the process that is used to produce the product. The time to implement a piece of code is a process metric on the development process.

Metrics play a critical role in controlling software development because they allow a project to be measured. Only then can the current state of the project be accurately assessed. However, as it will be shown in the next section, metrics are used in all aspects of software development and not just project management.

\subsection{2) Uses}

In this section, example uses of metrics are described. Again, these examples are strictly illustrative. They are only meant to show how metrics could be used - not which metrics to use. For object-oriented development, the recent book by [Lorenz and Kidd, 1994] should be consulted for suggestions of possible metrics. 
As it was discussed, metrics provide numerical measures for a software development project. This can be used to profile the project [Valett and McGarry, 1985]. A profile compares a metric's value across the entire project. From this, decisions can be made based on how the metric is distributed. For example, say the time to complete each phase in the project is measured. If it is found that the majority of time was spent in the testing phase, extra resources can be assigned to the testing phase because it takes the most time. Without the profile, the extra resources would have to be assigned without the benefit of supporting metric values. This could result in the resources being assigned to a phase that takes less time than the testing phase, which would limit the benefit to the project.

Another use of metrics is to monitor the project [Pfleeger, 1993]. To monitor a project, target ranges for certain metric values are set at the beginning of the project. During the duration of the project, the metrics are compared to their targets to make sure they are within the specified range. For example, a target range can be set for test coverage. After each class is tested, the test coverage metric must be above the target range. If it is not, additional testing should be done. This will ensure a standard consistency of testing across the project.

When a metric is correlated with another metric, prediction is possible [Kolewe, 1993]. For example, suppose a metric collected during the coding phase is shown to correlate with the number of defects found in the testing phase. By using the metric, an indication of code quality can be determined before the actual testing of the code. If a high number of defects is predicted for a piece of code, it can be re-written before resources are wasted on testing it. 
Metrics can also be used to give developers a qualitative evaluation of their work [Ince, 1990]. As an example, consider the code metric that is known to correlate with defect rate. By collecting the metric on their code, developers can get an indication of how defect free their code is.

Finally, the accuracy of estimates can be tracked with metrics [Dale and van der Zee, 1992]. Estimates are made at the beginning of the project. At the end of the project, the estimates can be compared to the actual values as collected by metrics. This can be used improve the estimating process. Furthermore, metrics can be used to provide estimates based on historical data. For example, the average number of defects per line of code can be calculated from past projects. Using this figure, an estimate of the number of defects in a current project can be determined by multiplying this ratio with the current number of lines of code.

\subsection{3) Costs}

Metrics offer many advantages, but how much overhead do they add to development? In a twelve year study on the use of metrics at one organization, the following results were found [Valett and McGarry, 1989]. Collecting the metric data took 2 to 3 percent of the total development costs. Processing the data look 5 percent of the costs and analyzing the data took 10 to 15 percent of the total costs. It should be noted that in this organization, much of the data was manually collected using forms that had to be entered into a database. Providing automated assistance for the collection and analysis of metrics will significantly reduce their overhead costs. 


\subsection{4) Analyzing}

The analysis of metric data is time consuming because it is like detective work. The main goals of metric analysis are to find unusual metric values, to identify trends of metric values, and to find relationships between metrics. To help with this, there are many tools and techniques available to analyze metric data. These will be discussed in the following sections.

\subsubsection{1) Mathematical tools}

Calculating the mean, median, and mode are probably the best known ways to analyze data [Kitchenham and Pickard, 1987]. Each of them work on a set of data points. The mean is defined as the numerical average of the values. It will give the single number that represents the average value of all values in the set. The median is the midpoint between the highest and lowest values in the set. It gives an idea of the range of values, not considering the distribution. Finally, the mode is defined as the most frequently occurring data value. It provides an indication of how the values are distributed.

The mean, median, and mode each attempt to provide a single number to represent all of the values in the data set. This makes them useful in identifying unusual metric values, otherwise known as outliners. This can be done by comparing a current metric value to the mean, median, or mode calculated from past values. If the current value is far from the mean, median, or mode then it may indicate the metric is in need of further analysis. For example, suppose that the average project value for time spent testing a line of code is $\mathbf{8}$ minutes. If a certain piece of code has a value of 2 minutes, then it may suggest this code is not well tested and has a high rate of defects. Note that in 
identifying outliners, all that can be said is that the value is an outliner and deserves further attention. It cannot be concluded with certainty that the value is good or bad.

While the mean, median, and mode attempt to summarize a data set, other methods are concerned with how the data set is distributed [Conte et al., 1986]. One of these methods is the standard deviation. A large standard deviation indicates that there are many values that are far from the mean. Skewness measures the symmetry of the distribution. A zero value for skewness indicates that the distribution is symmetric on both sides of the mean. Positive or negative values indicate that data is clustered on either the left or right of the mean. Finally, kurtosis measures the height of the distribution. The peek of the normal distribution is defined to have a kurtosis of zero. A positive or negative kurtosis indicates the distribution peeks higher or lower than the normal distribution.

It is important to know if a sample of data has a normal distribution because many of the more powerful statistical analysis techniques assume a normal distribution for the data being analyzed. Some of the techniques requiring a normal distribution are regression, analysis of variance (ANOVA), factor analysis, and chi-square [Conte et al., 1986]. Since metric data usually has a non-normal distribution, these techniques cannot be used unless the data is first transformed. Even then it is not guaranteed that transformation can make a data set follow a normal distribution [Fenton, 1991].

Regardless of the theoretical issues, it has been shown that the key for the acceptance of metrics in industry is to keep analysis simple [Anderson, 1992]. Only then will developers feel comfortable in performing metric analysis. This is for the best because the majority of developers will not be trained statisticians. Therefore, they will not understand how to use or interpret the results of the more sophisticated analysis 
techniques. For these reasons, these analysis methods will not be further investigated in this thesis.

\subsubsection{2) Graph based tools}

The next set of tools rely on visual means to analyze data. Graph based tools use the graph's ability to present a large amount of data in an easily understandable form to allow quick but powerful analysis.

A histogram is used to display the frequency of occurrence for a single variable [Robertson and Secor, 1986]. The $x$ axis contains the range of values for the variable. The $y$ axis contains the frequency for each value. An example of a histogram is shown in figure 4. Histograms are best used to display the distribution of the data sample. The histogram in figure is skewed to the left and therefore does not follow a normal distribution.

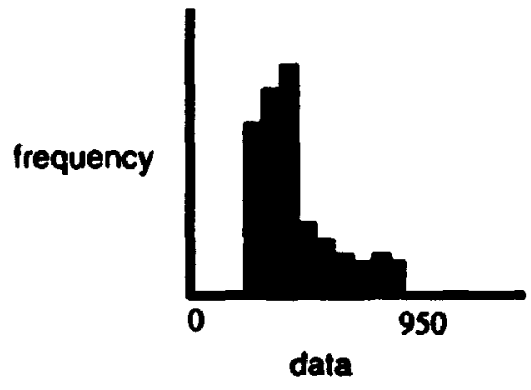

Figure 4: A histogram

Another graph used to display a single variable's distribution is the box plot [Myrvold, 1990] and [Fenton, 1991]. It is constructed as follows. The mean and median of the data set are calculated. A box is then drawn from the first quartile to the third quartile of the distribution (the first quartile is the median of all data points up to the 
median of the distribution and the third quartile is the median of all data points beyond the median of the distribution). The median is marked as a vertical line inside of the box and the mean is marked with a + symbol. The box length is multiplied by 1.5. From this value, the upper and lower tail values are calculated by adding and subtracting the first and third quartiles. The values of the upper and lower tails are truncated to the nearest data point. The tails are drawn projecting from the box. Finally, any data points outside of the tails represent outliners and are plotted individually. An box plot using the same data as in the histogram of figure 4 is shown in figure 5 .

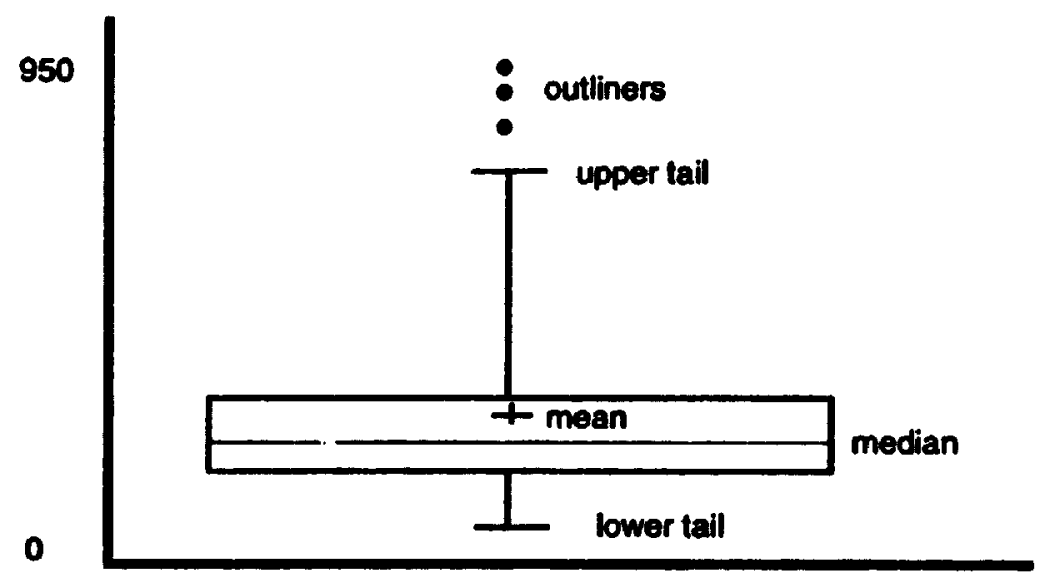

Figure 5: A box plot

The skewness of the data is shown by the lengths of the tails. With the lower tail being shorter than the upper tail, the data is more skewed to the lower values (the left side in the histogram). The position of the median in the box also shows the skewness of the distribution. A normal distribution would have equal length tails and a median in the center of the box.

The next type of graph, a scatter plot, displays two variables simultaneously [Kitchenham and Pickard, 1987]. The $x$ axis displays one variable, and the $y$ axis the other. An example would be to plot time spent testing verses number of defects found. 
Using the scatter plot, the relationship between the two variables can be determined. In the example, one would expect that as testing time increases, so does the number of defects found. The scatter plot can also be used to visually detect outliners. If in the example, one point is well below all of the others, it is an outliner. For this point, doing the specified testing found less than the expected number of defects. This may mean the testing was not thoroughly done and should be done again or that the code was of above average quality. This again illustrates how outliners just point out possible problems and further investigation is required to determine what the outliner actually means.

The last type of graph that will be discussed is called a multiple metrics graph [Pfleeger et al., 1992]. As the name suggests, it can be used to show an arbitrary nuniber of metrics at the same time. It is constructed by first drawing an inner and outer circle. The outer circle represents the maximum values for the metrics. The inner circle represents the desired values for the metrics. The circle is divided into slices. Each slice represents a metric and the size of each slice is representative of the relative importance of the metric. Each of the slices are normalized so their scales are equal. The values for each metric are indicated as points in each slice. The position of the point indicates the value of the metric. Each point is connected with a line to the intersection of the inner circle and the edges of the slice. An example multiple metrics graph with three metrics is shown in figure 6. 


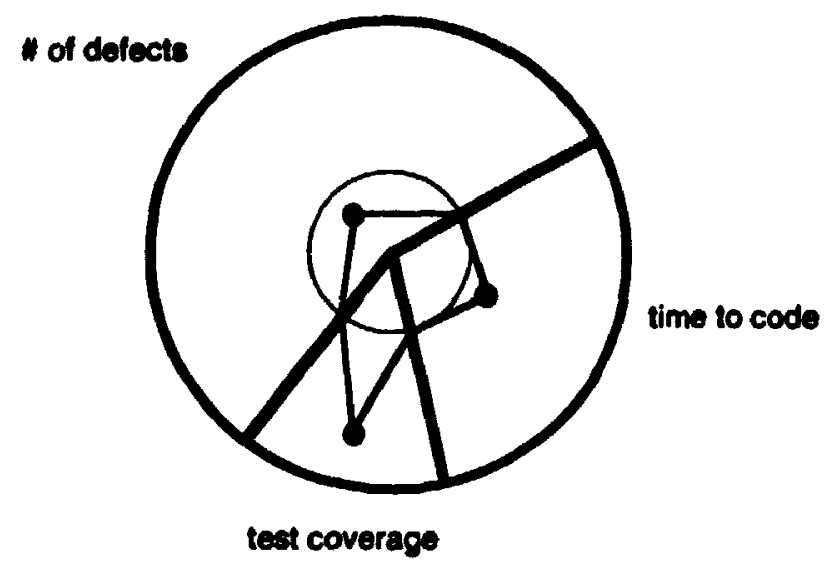

Figure 6: A multiple metrics graph

The graph in figure 6 shows three metrics, the number of defects, the time to code, and the test coverage as measured on a piece of code. From the sizes of the slices, it can be seen that the defect metric is the most important, followed by the time to code. and lastly the test coverage. The point representing the value of the defect metric is inside the inner circle. This means the value exceeded the goal (i.e. there were a very few number of defects). The time to code was close to the goal and the test coverage was far from the goal. From the relative area of each triangle with respect to the size of the slice it is contained in, it can be judged how well the metric met its goal. In the figure, the size of the triangle inside of the defect metric's slice is small in comparison to the slice. This confirms that the metric met its goal. On the other hand, the test coverage's triangle takes up a large portion of its slice. This indicates that the metric did not meet its goal. By viewing the area of the polygon created by the union of the triangles with respect to the area of the circle, an overall assessment of the metrics can be made.

From the multiple metrics graph, the following analysis can be performed. For the code the metrics were collected on, it is very important to have few defects. This goal 
was achieved. Since making good quality code takes more time, the low defect count must not come at the expense of the time to produce the code. This goal was not achieved, however the time to code was not too far off from its target. Finally, the amount of testing determines how many defects are detected. Therefore, a goal must be set for the test coverage to make sure an adequate attempt was made to find all of the defects in the code. This goal was not met. Apparently, high quality code was produced in an acceptable amount of time, but at the expense of testing. The code should be more thoroughly tested to uncover any additional defects. This example shows the power of multiple metrics graphs. They allow related metrics to be viewed together so their values can be analyzed in the proper context.

This concludes the discussion on analysis techniques. As it will be discussed in the next section, different people within a project have different objectives in using metrics. These objectives will dictate which metrics they find useful and how they will analyze the metrics.

\subsection{5) User's perspectives}

For the purposes of this discussion, three general roles within a project will be assumed [Grady, 1990]. The first role is that of the developer. These are the people who do the work resulting in the development of tic system. Developers need metrics to give an indication of the quality of the work they produce. This can encompass such measures as code quality, code understandability, code complexity, or testing adequacy. They will analyze these metrics to find ways to improve their work.

The second role is filled by the project manager. The project manager must coordinate the developers so that each of them is working on the correct tasks and the 
tasks are done according to schedule. Managers are interested in the project as a whole. They need metrics to identify trends and problem areas. The metrics must be analyzed to determine if the project is falling off of the schedule. Managers do not need to know the intricate details of individual pieces of work as developers do.

The last role is the public. These are people who are not directly involved with the project but still want to get an idea of how the project is going. The public will be interested in general project metrics that give an indication of the project's current state. The public only needs a general view of the project, and not the specific metrics that project managers use.

\subsection{6) Ensuring acceptance}

Given the different objectives of the people who use metrics in a project, difficulties may arise as to various members of the project accepting the metrics. The acceptance of metrics is important due to the time it takes to collect and analyze them. If people are not convinced of a metric's utility, they may provide inaccurate data or not bother to analyze the metric. As an example, consider the number of defects metric. Assume that after the testing phase, the number of defects are collected. If the owner of the code is given this metric and allowed to fix the defects, the metric should be seen as useful since it allows developars to improve their work. If, on the other hand, the metric is used by managers to assess the skill of the developer who wrote the code, the metric will not be accepted. The metric may even be falsely reported by the developer so he or she gets a good skill rating. Based on the experiences of organizations using metrics, the following recommendations can be made to help ensure the acceptance of metrics in a project. 
The first recommendation is to make sure everybody understands the need for metrics and can benefit from the metric's information [Pfleeger, 1993]. Metrics are traditionally viewed as a project management tool. Developers often have to expend the effort to collect them, but they do not get any use out of them. For a metrics program to be successful, everybody on the project must be able to use metric data to help with their work on the project.

Another recommendation is to keep the metrics manageable [Anderson, 1992]. This means collecting only a small number of metrics and keeping their analysis simple. Having too much data only leads to confusion and a cursory analysis. It is better to collect a few metrics that can be thoroughly analyzed. Analysis techniques must be kept simple because most project members will not have the theoretical background to use sophisticated statistical techniques. It must be made clear when and how the metrics are collected, how they are analyzed, and how they can be used to benefit the project.

Lastly, it should be stressed that the metrics are not going to be used to judge or criticize people [Grady and Caswell, 1987]. Without this philosophy, people will worry about how metrics make them look to others. This can cause people to alter their working habits to favorably affect the values of the metrics.

When metrics are used on a project, there are some other issues .., at are of concern. The most important one is that metrics are more than just numbers [Dale and van der Zee, 1992]. It is not enough to say that a metric, for example time to code, is to be collected on a project. The goal behind the metric must also be specified. This is because there can be many goals for a given metric and it is the goal that dictates how the metric is used and analyzed [Basili and Rombach, 1988]. For example, the time to code metric can have the goals of determining how much more productive developers are 
using a new development environment, the effect of adding more people to the project, finding the most productive developers so they can be given the hardest tasks, or tracking the project against the schedule. Each of these goals necessitates a different analysis procedure and application of the results to the project. With each metric, it should be understood what the intent of using the metric is, how it is analyzed, and how the analysis is applied to the project.

A related issue is integrating metrics with the development process [Shepperd, 1992]. Just as a metric may have many goals, a given metric can be collected at many different points in the procuss. For example, the number of defects metric can be collected before compilation, after compilation, before testing, after testing, before code review, or after code review. Depending on where it is collected, it will have different values. For metric analysis to be accurate, it must be certain that each metric value was collected at the same point. It would make no sense to compare the number of defects for two pieces of code when one was collected before testing and the other was collected after code review. This also applies to inter-project analysis of metrics. Two projects cannot be compared unless the metrics were consistently collected on both of them.

As a final word on metrics, something must be said with respect to the power of metrics. Metrics are important and provide useful information because they give numerical values to certain attributes. The problem that some people have in using metrics is that they try to equate a metric to a global property [Evangelist, 1988]. For example, they will equate code with a high number of branches as being complex and prone to defects [Grady and Caswell, 1987]. Although many metrics viewed together can give an indication of a global property, one metric alone cannot be said to be the sole predictor of the property. This is analogous to determining a person's health. It would be erroneous to base a person's health solely on their cholesterol level. Although a person's 
cholesterol level is an indicator of their health, it cannot be said to determine their health. However, if many of these indicators are taken together (age, heart rate, cholesterol level, exercise habits, and diet) a good picture of the person's health can be drawn. This is precisely the way in which software metrics are meant be used.

\subsection{7) Object oriented metrics}

The preceding discussion on metrics was kept general and could relate to metrics used on any type of project. While there has been a lot of work done in the area of structured metrics, metrics for object oriented projects are still actively being researched [Lorenz and Kidd, 1994]. In this section, aspects of object oriented development and object oriented languages that differentiate them from the structured paradigm will be discussed. Object oriented metrics must take these into account in order to provide accurate measurements and results.

Object oriented programs consist of interacting objects. This has two implications. The first implication is that the object becomes a fundamental unit of measure. Since objects contain state and behaviour, any metric relating to objects, must take this into account. For example, the size or complexity of an object depends on both its attributes and its methods. The second implication results from the fact that objects communicate with each other through messages. In an object oriented program, objects invoke services of other objects though messaging. This makes the flow of control more distributed across objects.

Tracing the flow of control is also complicated by dynamic binding (if the object oriented language is dynamically bound). This means that the object a message is sent to 
is determined at run time. Furthermore, due to polymorphism, many different objects can respond to the same message in different ways.

The last special feature of object oriented languages is inheritance. This allows state and behaviour of one object to be shared by another object. The subclassed object can add additional behaviour or override existing behaviour. As a result of inheritance, understanding a subclassed object necessitates understanding all of its superclasses.

With respect to the object oriented development process, the two defining features are incremental development and reuse. The incremental development style means an object oriented project is broken down into many repetitive micro-development efforts. Reuse has a effect on the time spent developing. The amount of code reused and the time it took to locate and understand the reused code must be taken into account. Additionally, the time to generalize code to be reusable must also be considered.

These aspects of object oriented technology mean that object oriented metrics have different properties to measure. Although some of the more general structured metrics can be applied to object oriented projects, most code specific metrics must be specially defined for object oriented languages. This will be further discussed in the section on relevant work.

\subsection{8) Discussion}

From the discussion on metrics, the following inferences can be drawn. First, in order for metrics to be used successfully on a project, they must be treated as more than just numbers. They have to have goals and analysis techniques associated with them. Only then can the metrics' data be accurate' : used to make decisions to benefit the 
project. Secondly, metrics collection must be integrated with the process model of the development process. In this way, it can be guaranteed that metrics are collected consistently throughout the project. Comparisons between metrics can then be made with high reliability. Taking these two points into consideration, it should be clear that the 1001 must include these ideas if it is to provide the maximum benefits of metrics. Exactly how the tool achieves this will be explained in sections 4 and 5 .

\section{7) Summary}

This concludes the presentation on background information. The preceding sections have given information on specific areas that Vision covers. In each section, it was shown what could be drawn from the information and applied to Vision. In the next sections, relevant work in the areas of process modeling and metrics will be discussed. The relevant work will provide further ideas for Vision. 


\section{3) Relevant work}

\section{1) Process modeling}

This section will describe several process modeling systems. The systems will be used to show a sample of some of the similar work being done in the area of process modeling. Many of the systems gave ideas for this research.

\subsection{1) Eureka Software Factory}

The Eureka Software Factory is an architecture for integrating CASE tools [Fernström et al., 1992]. The main drawback of CASE tools is that there is no one tool that will cover the entire lifecycle. For example, analysis and design tools do not handle testing. As stated in Fernström et al. [Fernström et al., 1992], ‘... no CASE vendor can support all the administrative and technical activities of a software enterprise. So CASE developers specialize, providing support for only part of the process.' In other words, no one company has the expertise or resources to create a tool to cover the entire lifecycle. Therefore, the CASE marketplace is made up of many niche tools that deal with specific parts of the lifecycle. The goal of the Software Factory is to provide an environment for integrating the various CASE tools. In this way, complete lifecycle coverage can be attained.

The Software Factory uses a process model to define how the development occurs. The process model specifies the tasks to do and which of the CASE tools are used to perform each task. When developers receive a task, they are provided with a description of the task, the appropriate tools and data to do the task, and a way to tell the 
Software Factory when they have completed the task. By having the developers follow the process model and perform their tasks, the Software Factory can coordinate the use of the appropriate CASE tools for a given task.

The main idea that the Software Factory provided for this research was the need for integration with other tools. Vision will implement process modeling and metrics features. However, it may become desirable to use the process model to invoke other tools like the Software Factory does. This was done through the use of generic objects and will be detailed further when Vision is described.

\subsection{2) Project Master Database}

The Project Master Database (PMDB) is a tool for constructing process models [Penedo and Shu, 1991]. Its defining feature is the large database of process model components it supplies. The database contains many objects. Some examples of the objects in the database are test cases, products, developers, or documents. The objects can have attributes like id or status. Specific relationships are used to tie objects together. For example, a test case object tests a software component object and causes a problem report object. Objects have operations associated with them that are invoked by the reception of events. An example operation is to change status after receiving the update event.

Using this library, an appropriate process model it i "l i. The model will show the objects and attributes and how the objects are related. When the model is run, events will trigger operations to update the model. In this way, complex process models can be quickly developed by using the existing library components. 
The power of having a library of process model components is the main idea garnered from the PMDB. Vision will take a slightly different approach. It will not have a library of pre-defined components. It will, however, allow process models and components of process models that were developed by users to be stored in a library. This will let users build up a library of process models and reuse them appropriately.

\subsection{3) Visual Process Language}

Vist!dl process tanguage (VPL) is a visual language used to represent process models [Shepard et al., 1992]. The language consists of nodes that are linked together to form a graph. The graph represents the process model and specifies the control flow in order to enact it. It has the following node types. The start and finish nodes mark the start and end of the process model. A task node represents some work to be done (e.g. write test cases). Decompose and recompose nodes are used to break up and combine work. For example, a code module can be decomposed into many sub-modules to be implemented by different developers. Once completed, they are recomposed into one module again. Split nodes are used to duplicate control flow. A piece of code can be copied so that several different developers can examine it to prepare for a code review. Merge nodes are used to combine multiple control flows into one. After each of the developers has examined the code, their paths will be merged so they can all met to perform the review. Branch nodes selectively choose an output path based on some criteria. For example, after exiting the testing task, a branch will be made depending on whether the testing was successful. Finally, procedure nodes are used to aggregate related nodes together. The nodes inside of the procedure node form a graph. Procedure nodes can be used to represent complicated tasks or sub-processes. 
Once the process model is represented in VPL, it can be enacted. Enactment begins at the start node and flows through the graph until it reaches the finish node. While traversing the graph, the appropriate tasks are performed as specified by the process model.

A visual graph based language was also used to represent the process in Vision. It has many analogous node types to those of VPL. The main ideas for how to represent the process model came from VPL and Process Weaver. Process Weaver is discussed in the next section.

\subsection{4) Process Weaver}

Process Weaver is an environment to give automated process support to a software development project [Fernström, 1993]. It has the following tools. A graphical editor allows the construction of process models, a model instantiation tc $>l$ is used to create instances of process models to be used in a project, the process engine enacts the process models, an agenda displays relevant tasks, and a process viewer is used to display enacied process models.

To construct a process model, the graphical editor is used. The process is first broken down into a hierarchy of sub-processes. This allows large processes to be abstracted into smaller sub-processes. Each sub-process has a cooperative procedure associated with it. This represents the steps to perform the sub-process. The steps are represented in a visual graph like language. Similar to VPL, there are start, end, condition (branch), and action (task) icons. Each action icon represents a unit of work to be done. When the action icon is enacted, it opens up a work context window. This window displays a text description of the work to be performed, a notepad where 
personal information can be entered, icons to run the appropriate tools to do the task, and control buttons to tell the tool the status of the task (e.g. started, on hold, completed).

After a process model has been created, it is instantiated. This involves initializing the process model's parameters with the appropriate values. For example, the tasks that a given developer are to perform have to be specified. Each developer on the project interacts with Process Weaver through the agenda tool. The agenda tool acts as a mail box for receiving assigned work contexts. From the agenda tool, the work context can be opened and the specified task performed. The process models created for the project are viewed with the process viewer and enacted with the process engine.

The important ideas provided by Process Weaver are the use of a graphical editor to create processes, the manner in which tasks are represented, the agenda tool to hold work contexts assigned to a developer, and the viewer to view the enacted process models.

\subsection{5) Hakoniwa}

Hakoniwa is another system designed to provide process support for projects [lida et al., 1993]. Similar to Process Weaver, it allows graphical process models to be constructed and enacted and it presents each developer with a list of tasks they are assigned. The interesting part is how the model is enacted. Hakoniwa uses messages to synchronize the various nodes in the graph representing the process model. For example, to start a node, a start message is sent to the node. The node then performs its purpose and sends a start message to the next node in the graph. This makes the enactment object-like since it uses messages and treats nodes as objects. This type of enactment was used in Vision because it naturally fit into the object oriented implementation of the tool. 


\subsection{6) Softman}

Just like Hakoniwa and Process Weaver, Softman also provides process support through the use of graphical process models [Mi and Scacchi, 1992]. Softman differentiates itself from the previous systems by the importance it places on roles. It assumes that a project will have manager and developer roles. Depending on the role of the person using the tool, different information is required.

The information provided by the developer role is similar to the information given by the developer task list in the other systems. It presents the developer with a list of tasks they have been assigned and provides an interface to the tools they need to perform these tasks. The developer can also be given a process model to specify how the tasks are to be done.

People filling the manager role have the objective of managing the resources assigned to the project. They need information as to resource utilization and the current status of an activity. They also have to be able to assign work to specific developers. In Softman, this is done through the managers interface. For a given task assigned to a given developer, the managers interface can show the individual steps that comprise the activity. This is displayed as a hierarchical tree. The task can be tracked by showing the process model that tells the developer how to do the task. This will show the point in the process where the developer is currently working. Tables are used to summarize resource utilization and task information for the entire project. Finally, the managers interface can be used to create tasks and assign them resources. The new tasks will be displayed in the task list of the developer who will perform them. 
The of roles is the main contribution of Softman. As it will be shown later, Vision will also have managers and developers roles. This gives Vision added power since it recognizes and supports the different objectives of different people on a project. It allows Vision to provide equal support for both project management and project development.

\subsection{7) Amadeus}

Amadeus is the last process modeling system that will be discussed [Selby et al. 1991]. Similar to the other systems, it provides process support for projects. The main difference with Amadeus is that it integrates metrics with the process model. In Amadeus, the process model specifies both the tasks to perform and the metrics to collect. This allows metrics to dynamically guide the process. For example, after a coding phase, metrics can be collected to give the number of revisions and cyclomatic complexity ${ }^{6}$ for the code. If there were many code revisions and the cyclomatic complexity is high, there is a greater chance of the code containing defects than if the values were low. Therefore, if the metric values are above a certain range, the code should be subjected to a very detailed test suite. If the values are below the range, it can be tested less stringently. This allows the project's limited resources to be concentrated on the highest risk components.

The use of metrics to pro-actively guide the process is a very powerful idea. It is incorporated into Vision through dialog objects. This will be discussed further in the section describing the tool.

\footnotetext{
6Cyclomatic complexity is a popular structured metric that attempts to measure the complexity of code by counting the number of control branches [Fenton, 1991].
} 
This concludes the overview of relevant process modeling systems. In the next section, relevant research on metrics is presented.

\section{2) Metrics}

As it was previously stated, there has been a lot of work done in the area of structured metrics but not much in the area of object oriented metrics. Due to the differences between structured and object oriented languages and development, most of the work in structured metrics work cannot be directly applied to object oriented metrics. However, there are a few general metrics that apply to any development effort. These include measures of productivity and quality.

\subsection{1) Productivity}

The most common way to measure productivity is by the number of lines of code produced per developer month [Factor et al., 1988]. Although this is not perfect, it has become fairly standard for use on structured projects. Its main drawback is that it only considers the developer as a determinant of productivity. It ignores other influences such as the caliber of management, organizational structure, software tools used, and methodology followed [Myers, 1989].

The applicability of lines of code per developer month as applied to an object oriented project is unknown. While the time required to complete a task is a valuable measure, relating it to lines of code may not be useful. This is because lines of code is probably too fine grained. It may be better to use methods or classes as the base unit of measure. Furthermore, the effects of reuse and generalization must also be considered. 
A definitive way to measure object oriented productivity is still the subject of active research.

\subsection{1) Quality}

The main determinant of quality is the number of defects that are found. Although there are other factors such as cost of the system, time to delivery, and user friendliness, most quality metrics focus on defects [Inglls, 1986]. The definition of defect can vary, but usually it is taken to mean a failure of the software to work properly or perform according to specifications.

In a similar manner to productivity, quality is usually related to a base unit of measure. In structured projects, quality is typically defined as the number of defects per thousand lines of code [Conte et al., 1986]. This allows pieces of code of different sizes to be compared with respect to quality. However, just as basing productivity on lines of code was of questionable use in object oriented projects, so is basing quality on lines of code. It is probably better to base it on some measure of a class' size.

Although it is desirable to find defects through testing, it is even more desirable to predict defects. This can be used to pinpoint defect prone code. Once this code has been found, it can be re-written or subjected to greater testing. Some metrics that have been correlated with defect rate are test coverage and number of code changes [deMarco, 1982]. For example, if only a small percentage of the code has been tested it can be said to have a low measure of test coverage. This means the untested portions of the code may contain defects that will show up at a later time. Code with low test coverage can therefore predict an increased chance of defects. The number of code changes metric works on the principle that changes to code can introduce bugs to previously working 
features. Code with a high number of changes is therefore at a higher probability of containing defects. In theory, these two metrics are a good indicator of defects. However, for any given project they should be tested to see how well they actually predict defects.

\subsection{3) Object oriented metrics}

There have only been a handful of papers published on object oriented metrics. Each of these paper's contribution will be discussed below.

Probably the most well known paper is by Chidamber and Kemerer which was published in OOPSLA '91 [Chidamber and Kemerer, 1991]. In it, they propose six object oriented metrics. Also included is a good presentation on the background theory behind the metrics. The metrics are summarized below.

Weighted methods per class (WMC) is calculated by multiplying each method by its complexity and summing the results. This is used to measure the size of the class. The size can be related to the time it takes to develop and maintain the class. Furthermore, large classes with many methods have a greater impact on subclasses since the subclasses inherit all of the methods. The metric can also indicate how reusable the class is. Classes with too many methods are more likely to be application specific and not reusable.

Depth of inheritance tree (DIT) is defined as the number of parent classes a given class has. This measures how many classes can potentially provide state and behaviour to a class. Classes with a high depth of inheritance are more likely to inherit 
attributes and methods. This makes them more complex to design and understand because they depend on other classes.

Number of children (NOC) is the number of immediate subclasses a class has. It measures how many other classes will inherit from the class. Classes with a large number of children should be well tested because many other classes will directly use its behaviour.

Coupling between objects (CBO) counts the number of methods that a given class uses of another class. Method coupling by inheritance is not included in this count. Coupling between objects measures the degree that one class depends on another class. It is not good to have two classes extensively coupled since it limits the reusability of one class without the other. It also makes the coupled class more sensitive to changes in the other class.

Response for a class (RFC) is defined as the union of all methods in a class and all methods called by methods in a class. This measures the communication with outside objects. Having a high RFC is not good because many other methods must be understood to understand how the methods in the class work. This makes testing, debugging, and maintenance more difficult.

Lack of cohesion methods (LCOM) measures the number of groups of methods that use common instance variables. It assumes that if two methods access the same instance variable, they are similar in function. Having a low cohesion value (very few methods using similar instance variables) is not good because it does not promote encapsulation. When the cohesion is low, the class should be split into two or more subclasses. 
In their paper Chidamber and Kemerer formally evaluate the metrics against a set of desirable properties metrics should have. However, they do not provide any empirical evidence that the metrics are accurate indicators of the properties they claim to measure.

The research by Chidamber and Kemerer as further extended by $\mathrm{Li}$ and $\mathrm{Henry}$ [ $\mathrm{Li}$ and Henry, 1993]. In it, they try to find which metrics are predictors of maintenance effort. They use all of Chidamber and Kemerer's metrics (except coupling between objects) as well as proposing some additional metrics.

$\mathrm{Li}$ and Henry were especially interested in the types of coupling between classes. Coupling is defined as two classes acting on or requiring each other. They identified three such types and defined metrics to measure the extent of the coupling. The first type of coupling is coupling by inheritance. It reflects the fact that inheritance allows classes to share state and hehaviour. It is measured by Chidamber and Kemerer's depth of inheritance tree (DIT) and number of children (NOC) metrics. The second type of coupling is coupling through message passing. If one class sends a message to another class, the first class is coupled to the second class. This is because the first class needs the second class in order for it to function. Li and Henry measure this type of coupling through their message passing coupling (MPC) metric. It is defined as the number of send statements in a class. The third type of coupling is coupling through abstract data types. This type of coupling occurs when one class contains a reference to another class in its state variables. This is measured by the data abstraction coupling (DAC) metric. It is calculated by counting the number of state variables referencing other classes.

$\mathrm{Li}$ and Henry also defined three additional metrics. The number of methods (NOM) measures the number of local methods in a class. This is used to get a measure 
of how complex the class' interface is. The higher the number of methods, the more methods there are in the class' interface. SIZE1 measures a class' size by counting the number of source code lines in the class. SIZE2 measures size by counting the number of attributes and local methods in a class.

Using these metrics, $\mathrm{Li}$ and Henry ran experiments to measure the number of lines of code that were changed as a result of maintenance. They hypothesized that some or all of the metrics could be used to predict which classes would have a high number of changes. They found that the following metrics were strong predictors of maintenance effort: DIT, NOC, MPC, RFC, LCOM, DAC, WMC, and NOM.

In a paper by Coppick and Cheatham, the researchers attempt to apply two popular structured metrics to object oriented languages [Coppick and Cheatham, 1992]. The metrics in question are Halstead's software science metrics and McCabe's cyclomatic complexity. Halstead's metric is thought to predict programming effort by measuring size attributes of the program [Fenton, 1991]. McCabe's metrics is supposed to predict defects by measuring the complexity of code [Fenton, 1991]. Although their small sample of data supports the validity of these metrics in an object oriented language, more work must be done before the claims can be fully justified.

Chen and Lu attempt to find object oriented design metrics that would predict class complexity [Chen and $\mathrm{Lu}, 1993$ ]. They defined eight metrics that could be calculated from the design specification for a class. These inetrics are described below.

Operation complexity is a measure of a class' method complexity. Each method is assigned a complexity rating. This is determined by subjectively rating a method's complexity (e.g. high, medium, low) and converting this rating to a number by using a 
table they supply. The results for all of the metrics are summed into one value for the class. Operation argument complexity measures the complexity of the arguments of the class' methods. Again, each argument for each method is assigned a value based on its type (e.g. boolean, array, file, etc.). Ti.e mappings between type and complexity value are given in another table. The results are added up to give a single value for the class. Attribute complexity measures the complexity of the class' state variables. Similar to the operation argument complexity metric, each state variable is assigned a complexity value and the results are summed.

The operation coupling metric measures the message coupling of a class. It is defined as the sum of the number of operations which access other classes and the number of operations which are accessed by other classes. The class coupling metric measures the coupling between classes. It is calculated by summing the number of access to other classes and the number of access by other classes.

The cohesion metric is analogous to Chidamber and Kemerer's lack of cohesion in methods (LCOM) metric. It also attempts to group methods into cohesive sets. However, instead of basing method cohesion on state variable references, the cohesion metric uses similarity of method arguments. The class hierarchy metric is defined as the depth of the class in the inheritance tree plus the number of subclasses plus the numi :r of direct super classes plus the number of local or inherited operations available to the class. It attempts to measure the inheritance coupling of a class. Finally, the reuse metric is defined as one if the class is reused and zero if it is not reused.

The researchers did an experiment where several object oriented designs were rated as to complexity. The metrics were correlated to this complexity rating to see which metrics could be said to predict complexity. They determined that the operation 
complexity, operation argument complexity, operation coupling, and class coupling metrics were accurate predictors of class complexity.

The final research that will be discussed is a masters thesis by Haaland [Haaland, 1992]. Haaland proposed many static, runtime, and evolutionary metrics for Smalltalk and ENVY/Developer (for an overview of ENVY/Developer see appendix 1). Static metrics can be calculated from the source code. Runtime metrics are calculated when code is being executed. Evolutionary metrics capture how the system changes over time.

For each metric, he calculated the average value based on several systems. There are ma: $v$ potentially useful metrics proposed in the thesis, however, no explanation as to how they can be applied to a project is given. The average values provide important data but they vary widely depending on the type of system they were collected on. The metrics used in by Haaland are summarized below.

\section{Method level}

- method length - measured by the number of byte codes, source code length, lines of code, and lines of comments

- method complexity - measured by the number of methods sent by a methid, number of arguments accepted, number of temporary variables, rumber of ENVY editions, number of public and private methods, and cyclomatic complexity

\section{Class level}

- inheritance - measured by the number of immediate subclasses, number of descendants of a class, and height of a class 
- state complexity - measured by the number of instance variainles and number of class variables

- reuse - measured by the number of inherited methods, normalized number of inherited methods, number of reused methods, number of refined methods, and number of new methods

- size - measured by the number of implemented methods and the number of public and private methods

- memory - measured by the average space in bytes occupied by instances and the number of instances of the class

\section{ENVY Application level}

- complexity - measured by the number of defined classes, number of extended classes, number of undefined classes, number of classes, number of public classes, and number of private classes

- coupling - measured by the number of prerequisite applications, number of all prerequisite applications, number of dependent applications, number of sub applications, number of all sub applications, and number of visible classes

- evolution - measured by the number of editions

- documentation - measured by the number lines of comments and number of lines of description

ENVY Class edition level

- complexity - measured by the number of methods, number of public methods, and number of private methods

- evolution - measured by the number of editions 
- documentation - measured by the number lines of comments and number of lines of description

\section{Runtime}

- method coverage - measured by the number of methods that were executed and the number of times a method was executed

- run time stack - measured by the method collaborator metric and class stability metric

\section{Evolutionary}

- change - measured by the number of added, reused, and removed classes

For a more complete description on how the metrics were collected and what their average values were, Haaland's thesis should be referenced. This marks the end of the discussion on metric research.

\section{3) Summary}

This concludes the review of relevant work. Many of the process modeling systems provided important ideas for Vision. The metrics research was used to select the best metrics to incorporate into Vision as examples illustrating the specification of metrics in general. As a result, this thesis can be viewed as the integration of the various ideas into one unified tool. The originality of Vision comes from the integration of process modeling and merics in the context of an object oriented software project. Currently, there is no known tool that provides process modeling and metrics support within an object oriented development environment. 


\section{4) Framework of Vision}

In this section, the general idea of Vision will be described. Specific details will be giver in section 5 which describes Vision. The main purpose in presenting the general framework of Vision is to show how it integrates the previous research.

\section{1) General overview of Vision}

As it was previously discussed, Vision's long-term goal is to help overcome the problems associated with the software crisis. Vision addresses this goal by providing project management, project development, and metrics features. The most important part of Vision is its process modeling capabilities. It is the process model that provides the common ground by which the three features are related.

In order to be used in a project, Vision must represent the project's software development process. This is done with a process model which specifies the various phases and steps in the process. A process model representing the possible phases of one iteration in a generic object oriented development process is shown in figure 7 (refer to appendix 2 for an example of using Vision to represent a specific process).

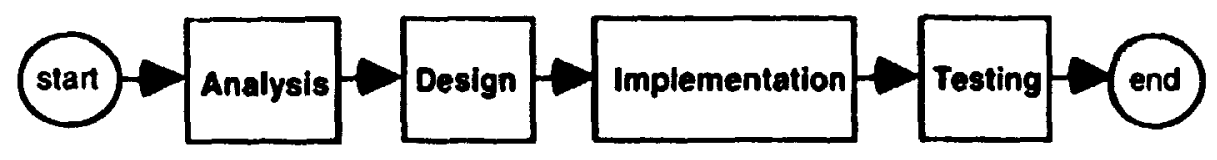

Figure 7: A generic process 
The process depicted in the figure has analysis, design, implementation, and testing phases. These phases are merely illustrative. For example, a prototyping phase could be added and the testing phase could be pervasive to analysis, design and implementation. Furthermore, each of these phases normally contains additional details as to what steps to perform in the phase. For this discussion, these steps are not important and are therefore not shown.

The circles in figure 7 represent the starting and ending point of the process model. The process model by itself is not iterative because it does not have a re-entrant loop from the testing phase back to the analysis phase. As it was discussed in section 2.2, object oriented development is characterized by a spiral which keeps iterating over the phases until the development is complete. Since the process model is figure 7 is not iterative, it represents one cycle of development or one loop of the spiral.

Object oriented development projects are typically composed of a finite number of cycles or loops through the spiral. This iteration is represented in Vision as follows. For each cycle, a separate copy of the process model is created. Therefore, by sequentially lining up many process models, an 'unwound' spiral is depicted. This is shown in figure 8. In each cycle, development occurs according to the process (this is shown as A-D-I-T for analysis, design, implementation, and testing). The entire project is made up of 4 development cycles. 


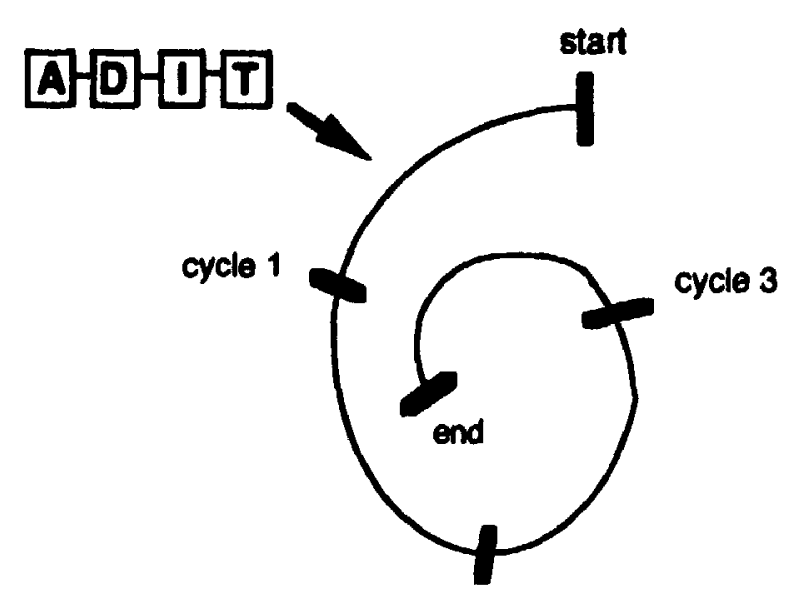

cycle 2

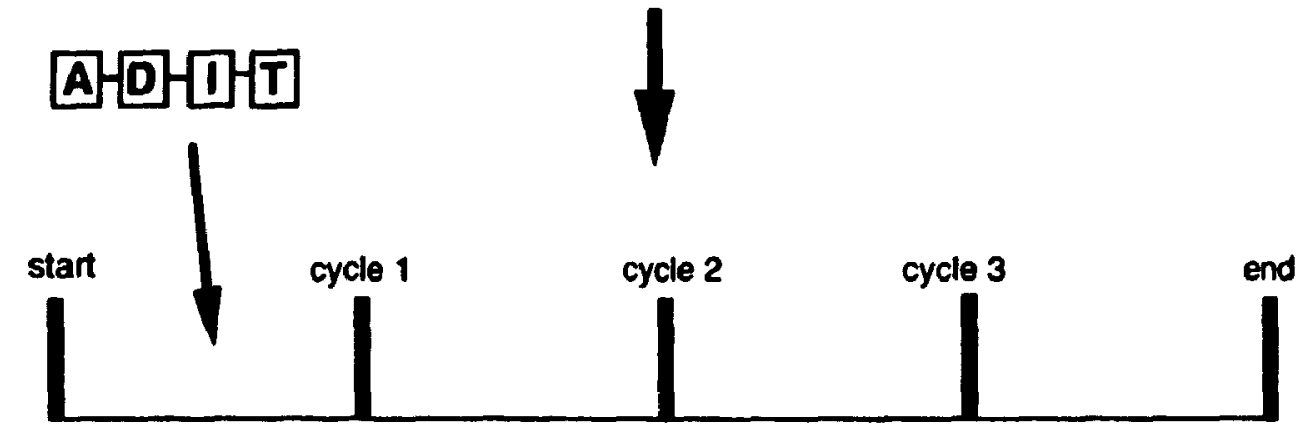

Figure 8: Unwinding the spiral

Within a development cycle, a team of developers implements a small portion of the overall functionality of the system they are creating. The features that will be implemented in a cycle are broken into tasks. Each task is assigned a developer to complete the task. Tasks represent trackable units of work and each one has a separate copy of the process model associated with it. Some examples of tasks in a project that involves developing a word processur are to do the spell checker, to allow files to be saved, or to provide on-line help. Note that since there are many tasks associated with a cycle, each cycle contains many separate copies of the process model that are being performed in parallel. Conceptually, however, the cycle still represents one iteration through the process model. 
From a developer's viewpoint, each developer has a list of tasks they have been assigned. Since a task references a process model, a developer can follow the process model to get assistance as to how to perform the task. By keeping the process model up to date as to which step in the process they are currently working on, it is possible for developers to communicate their progress.

From a manager's viewpoint, a manager has access to each task that is being performed in the cycle. By viewing the process model associated with a developer's task, the manager can find out which step of the process they are working on. This gives managers accurate tracking of tasks.

Within the process model, the places to collect metrics are specified. Since the process model is being kept up to date by the developer performing the task, the metrics can be automatically collected and stored. Therefore, both managers and developers can use a process model to access metric data. This data can be analyzed to help them with their respective work.

In this fashion, Vision supports project tracking, development assistance, and metrics usage within a project. This is the general framework by which Vision operates. In the next section, the previous research that Vision builds on is summarized.

\section{2) Previous research used in Vision}

In table 2, the ideas for Vision are attributed to the existing research that was described in sections 2 and 3. 


\begin{tabular}{|c|c|c|}
\hline Idea & Reason for using & $\begin{array}{l}\text { Source / section } \\
\text { describing }\end{array}$ \\
\hline \multicolumn{3}{|l|}{ General ideas } \\
\hline $\begin{array}{l}\text { Vision specifically } \\
\text { designed for } 00 \text { projects }\end{array}$ & $\begin{array}{l}\mathrm{OO} \text { is accepted in industry and is seen } \\
\text { as the future of software development. }\end{array}$ & Section 1.3.1 \\
\hline $\begin{array}{l}\text { Vision assists project } \\
\text { tracking, development, } \\
\text { and metrics }\end{array}$ & $\begin{array}{l}\text { These areas have an important impact } \\
\text { on the success of a project but have not } \\
\text { been well researched. }\end{array}$ & Section 1.4 \\
\hline $\begin{array}{l}\text { Vision integrated with } \\
\text { the } O O \text { development } \\
\text { environment }\end{array}$ & $\begin{array}{l}\text { The } \mathrm{OO} \text { environment has many } \\
\text { development tools, but no project } \\
\text { tracking or metrics tools. These tools } \\
\text { are needed to support the software } \\
\text { engineering aspect of development. }\end{array}$ & Section 2.1 and 2.3 \\
\hline $\begin{array}{l}\text { Vision tracks tasks but } \\
\text { does not provide } \\
\text { traditional project } \\
\text { management features }\end{array}$ & $\begin{array}{l}\text { There are many good commercial } \\
\text { software packages that handle general } \\
\text { project management. It is better to } \\
\text { work with them rather than duplicating } \\
\text { their functionality. }\end{array}$ & Section 2.4 \\
\hline $\begin{array}{l}\text { Vision uses process } \\
\text { models to represent the } \\
\text { development process }\end{array}$ & $\begin{array}{l}\text { The iterative nature of } O O \\
\text { development means the same process } \\
\text { will be used over and over. Process } \\
\text { models provide a template by which to } \\
\text { track project tasks. }\end{array}$ & Section 2.2 and 2.5 \\
\hline $\begin{array}{l}\text { Vision represents the } \\
\text { traditional view of } \\
\text { metrics as goals, } \\
\text { questions, metrics, and } \\
\text { analysis }\end{array}$ & $\begin{array}{l}\text { Metrics must be treated as more than } \\
\text { numbers if they are to be successfully } \\
\text { used to benefit a project. }\end{array}$ & $\begin{array}{l}\text { Basili and Rombach, } \\
1988 \\
\text { Section } 2.6 \text { and } 5.5\end{array}$ \\
\hline $\begin{array}{l}\text { Vision integrates metric } \\
\text { collection with the } \\
\text { process model }\end{array}$ & $\begin{array}{l}\text { This allows metrics to be collected } \\
\text { automatically and consistently in } \\
\text { projects. }\end{array}$ & $\begin{array}{l}\text { Amadeus } \\
\text { Section } 2.6 \text { and } 3.1 .7\end{array}$ \\
\hline
\end{tabular}




\begin{tabular}{|c|c|c|}
\hline Idea & Reason for using & $\begin{array}{l}\text { Source / section } \\
\text { describing }\end{array}$ \\
\hline \multicolumn{3}{|l|}{ Specific features } \\
\hline $\begin{array}{l}\text { Vision is integrated in a } \\
\text { development } \\
\text { environment containing } \\
\text { VisualWorks and } \\
\text { ENVY/Developer }\end{array}$ & $\begin{array}{l}\text { VisualWorks Smalltalk was chosen } \\
\text { because it is the best of the Smalltalks } \\
\text { with respect to robustness and cross } \\
\text { platform portability. ENVY/Developer } \\
\text { was necessary to provide support for } \\
\text { multiple developers. It also functions } \\
\text { as the database to store Vision's } \\
\text { process model and metric data. }\end{array}$ & Appendix 1 \\
\hline $\begin{array}{l}\text { Vision can use its } \\
\text { process model to } \\
\text { coordinate other tools }\end{array}$ & $\begin{array}{l}\text { Having one tool provide complete } \\
\text { lifecycle coverage is not feasible. By } \\
\text { representing the process, Vision has } \\
\text { knowledge of which tools are } \\
\text { appropriate at a given time. }\end{array}$ & $\begin{array}{l}\text { Software Factory } \\
\text { Section 3.1.1 }\end{array}$ \\
\hline $\begin{array}{l}\text { Vision has a library of } \\
\text { process models and } \\
\text { process components }\end{array}$ & $\begin{array}{l}\text { Storing process models allows them to } \\
\text { be edited and reused. Storing process } \\
\text { components makes it easier to build } \\
\text { new models by reusing existing } \\
\text { components. }\end{array}$ & $\begin{array}{l}\text { PMDB } \\
\text { Section 3.1.2 }\end{array}$ \\
\hline $\begin{array}{l}\text { Vision represents the } \\
\text { process model in a visual } \\
\text { graph }\end{array}$ & $\begin{array}{l}\text { A visual graph representation it easy to } \\
\text { enact the model by following the links } \\
\text { in the graph. }\end{array}$ & $\begin{array}{l}\text { VPL and Process } \\
\text { Weaver } \\
\text { Sections } 3.1 .3 \text { and } \\
3.1 .4\end{array}$ \\
\hline $\begin{array}{l}\text { Vision has nodes in the } \\
\text { graph corresponding to } \\
\text { start, end, split, merge, } \\
\text { branch, and activity }\end{array}$ & $\begin{array}{l}\text { These nodes represent a minimum set } \\
\text { of constructs that are necessary to } \\
\text { represent the process model in a graph. }\end{array}$ & $\begin{array}{l}\text { VPL and Process } \\
\text { Weaver } \\
\text { Sections } 3.1 .3 \text { and } \\
\text { 3.1.4 }\end{array}$ \\
\hline $\begin{array}{l}\text { Vision has a graphical } \\
\text { editor to construct } \\
\text { process models }\end{array}$ & $\begin{array}{l}\text { This makes it easy for anybody to } \\
\text { create a new model or modify an } \\
\text { existing one. }\end{array}$ & $\begin{array}{l}\text { Process Weaver } \\
\text { Section 3.1.4 }\end{array}$ \\
\hline
\end{tabular}




\begin{tabular}{|c|c|c|}
\hline Idea & Reason for using & $\begin{array}{l}\text { Source / section } \\
\text { describing }\end{array}$ \\
\hline \multicolumn{3}{|l|}{ Speciffc features } \\
\hline $\begin{array}{l}\text { Vision has a work } \\
\text { context associated with } \\
\text { an activity }\end{array}$ & $\begin{array}{l}\text { This provides the developer with } \\
\text { information on what to do and a } \\
\text { mechanism to keep the tool up to date } \\
\text { as to the status of the activity. }\end{array}$ & $\begin{array}{l}\text { Process Weaver } \\
\text { Section } 3.1 .4\end{array}$ \\
\hline $\begin{array}{l}\text { Vision uses message } \\
\text { sends between node } \\
\text { objects to enact the } \\
\text { process model }\end{array}$ & $\begin{array}{l}\text { Communicating by messages fits } \\
\text { nicely with the object oriented } \\
\text { implementation of Vision. }\end{array}$ & $\begin{array}{l}\text { Hakoniwa } \\
\text { Section 3.1.5 }\end{array}$ \\
\hline $\begin{array}{l}\text { Vision differentiates } \\
\text { between manager and } \\
\text { developer roles }\end{array}$ & $\begin{array}{l}\text { This recognizes that different roles } \\
\text { require different information. }\end{array}$ & $\begin{array}{l}\text { Softman } \\
\text { Section 3.1.6 }\end{array}$ \\
\hline $\begin{array}{l}\text { Vision has an interface } \\
\text { to view process models } \\
\text { assigned to developers }\end{array}$ & $\begin{array}{l}\text { This allows managers to track the } \\
\text { progress of each developer through the } \\
\text { process model. }\end{array}$ & $\begin{array}{l}\text { Process Weaver and } \\
\text { Softman } \\
\text { Section } 3.1 .4 \text { and } \\
3.1 .6\end{array}$ \\
\hline $\begin{array}{l}\text { Vision presents each } \\
\text { developer with their } \\
\text { assigned tasks }\end{array}$ & $\begin{array}{l}\text { This allows developers to concentrate } \\
\text { on the tasks they have been assigned. }\end{array}$ & $\begin{array}{l}\text { Process Weaver and } \\
\text { Softman } \\
\text { Section } 3.1 .4 \text { and } \\
3.1 .6\end{array}$ \\
\hline $\begin{array}{l}\text { Vision allows metrics to } \\
\text { proactively guide the } \\
\text { process }\end{array}$ & $\begin{array}{l}\text { This makes immediate use of metric } \\
\text { results to tailor the process to the } \\
\text { product produced by the process. }\end{array}$ & $\begin{array}{l}\text { Amadeus } \\
\text { Section 3.1.7 }\end{array}$ \\
\hline
\end{tabular}

Table 2: Previous research providing ideas for Vision

By integrating the best ideas of previous research, Vision has a sound foundation. This is $\mathbf{b}$ cause the ideas in the previous research have been well refined, tested, and validated as a result of the research. In the next section, Vision is described. This will show how Vision uses the previous research to realize the framework that was outlined in section 4.1. 


\section{5) Description of Vision}

In the following sections, Vision will be described. To begin, an overview of the context in which Vision is used will be presented. This is followed by a general description of Vision's features. Subsequent sections will give a more detailed description of each feature. To finish, Vision's benefits will be given.

\section{1) Organizational context}

As it was previously discussed, Vision was developed for Newbridge Networks Corporation. In this section, the organizational context that existed before Vision was developed will be described. This will be used to show areas that need improvement and will motivate the need for Vision. Note that although the Vision was developed for Newbridge, the organizational context that is assumed is kept general. This makes Vision applicable for use at other organizations because the situation at Newbridge is an instance of the general issues that any software development organization has to address.

The typical organization that will use Vision is as follows. The organization will have a well defined and documented process that specifies how software is developed. They will also believe in the use of metrics to monitor the process and product. Their goal will be to produce high quality software on budget and according to schedule.

The organization's development projects can vary in size. A project will, however, have many developers working cooperatively. Their development language will be ParcPlace VisualWorks Smalltalk. OTI's ENVY/Developer will provide configuration management. Managers will use a project management software package 
like Claris' MacProject, to create and maintain the project plan. The project plan will show the tasks in the project, who they are assigned to, how long they are estimated to take, and their current status. The project management application will handle resource allocation, tracking, and report generation on the tasks in the project plan. These tasks will be medium-grained in size and take under twenty days to complete.

Each developer is assigned a list of tasks. Every two weeks, they give their manager revised estimates of the time spent and the percent complete for each of their tasks. The manager uses this information to update the project plan.

\section{2) Goals}

Given this organizational context, the following observations can be made. With respect to project management, time and resources on a task granularity are well tracked. However, tasks can take up to twenty days so tracking within a task is not possible. Therefore it is desirable for tasks to be tracked at a finer level, but without adding the additional overhead of breaking tasks into smaller units of work.

With regards to development, the process is well documented on paper. What is lacking is some form of automated guidance to help developers follow the process. Furthermore, evolution and change of the process must be done through paper documents. There is no easy way to define a new process and distribute it to developers to try out.

In a similar manner, metrics are also documented on paper. There are a set of separate programs to collect the metrics, but no integrated tool to define, collect, and 
analyze them. Any new metrics must also be proposed on paper so there is no quick way to design, collect, and analyze a new metric.

Summarizing from these observations, some high level goals that Vision must address are given below.

- have an on-line process model

- developers will get process assistance

- managers will get finer tracking

- have metrics integrated with the process model

- will provide an environment to define, collect, and analyze the metrics

- have the tool seamlessly integrated with the development environment

- will make the tool's use as transparent as possible

\section{3) General overview}

At an abstract level, Vision can be viewed as consisting of five different subsystems. These are shown in figure 9.

The process modeling sub-system is responsible for the process modeling aspect of Vision. It consists of an editor to create process models and a browser to store and retrieve the models. The metrics sub-system handles metrics and has a browser to create, retrieve, and store metrics. Together, the metrics and process model can be used to represent a process which defines how to perform a task. The management sub-system allows tasks to be created and assigned to developers. The development sub-system lets 
each developer view the tasks they have been assigned. Finally, the enactment subsystem is used to view the status of the process models and the values of the metrics.

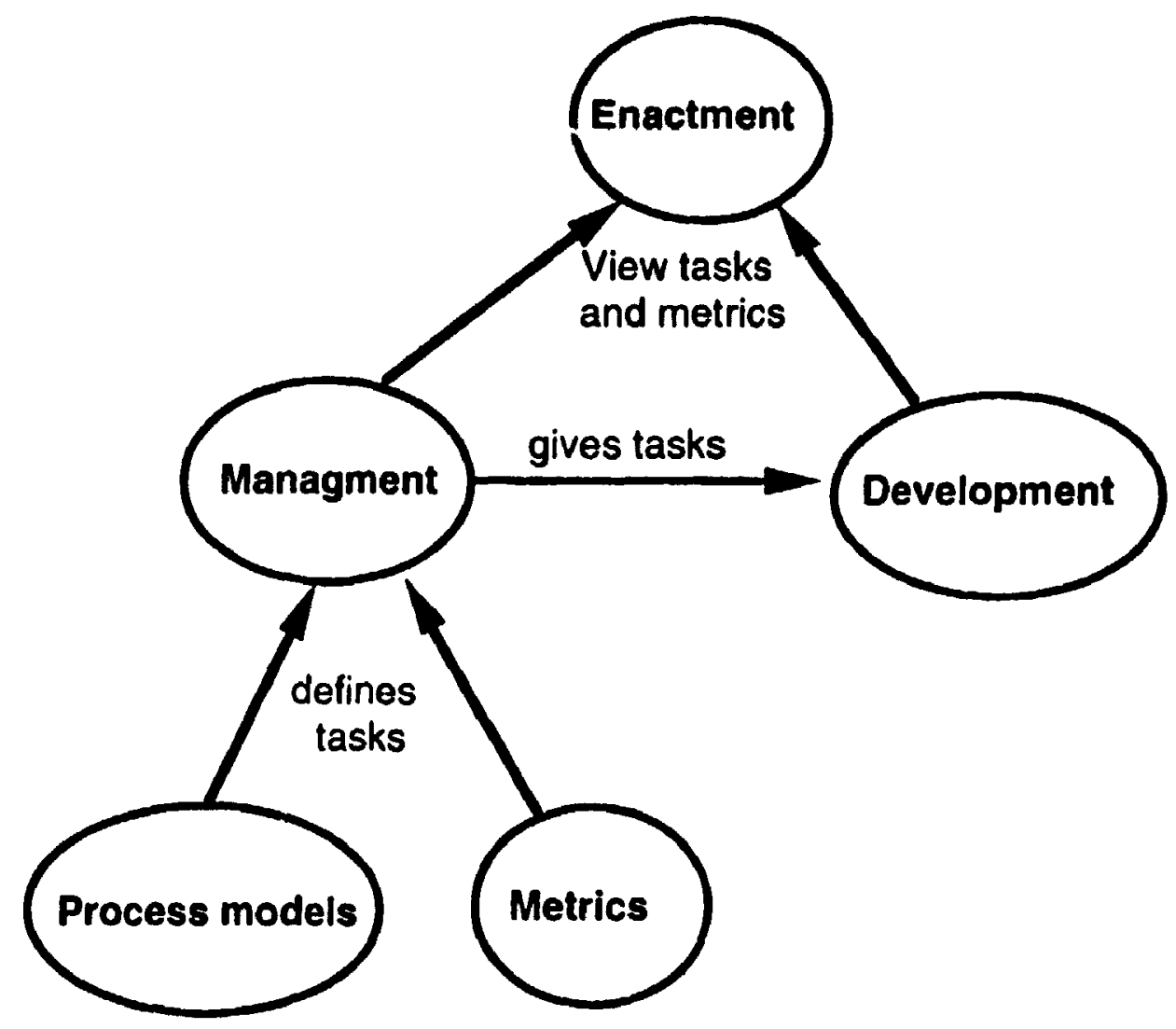

Figure 9: The sub-systems of Vision

The specific windows that comprise Vision are shown in figure 10. Each of these windows and sub-systems are further described in the following sections. The pictures of Vision that are shown represent actual screen snapshots from Vision. Note that the description will be high level and will only cover the main features. For a specific explanation of each feature of Vision, a reference manusi in the form of on-line help is available. A brief overview of the implementation of Vision is provided in appendix 3. 


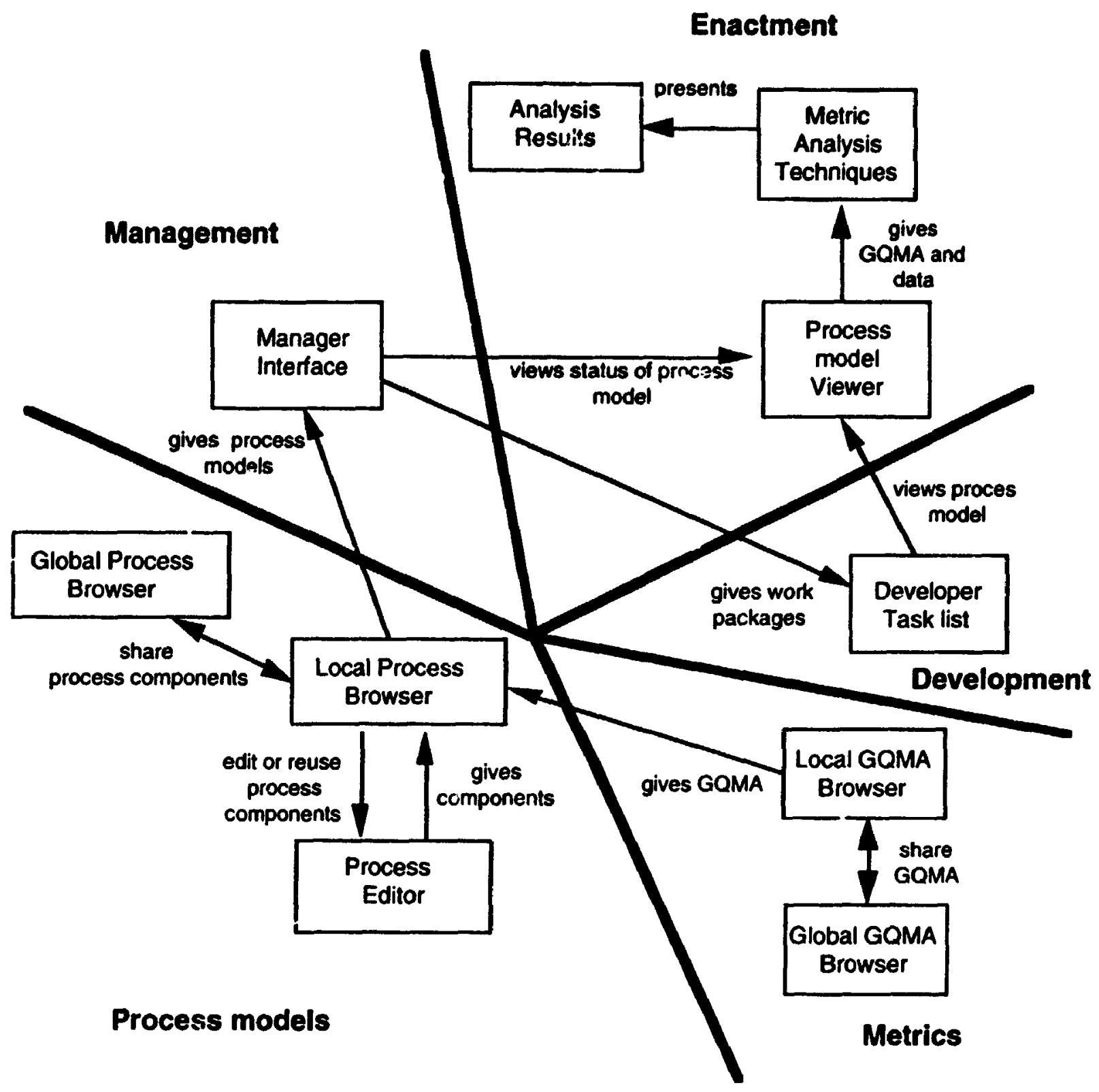

Figure 10: The windows of Vision 


\section{4) Process modeling}

The process modeling sub-system is made up of the process editor, local process browser, and global process browser. As shown in figure 10, the process editor is used to create process components. Process components can either be entire process models or specific objects in process models. The components are stored in a local library and are viewed using the local process browser. Components can be retrieved from the browser to be edited or reused. The global browser is an interface to the global process library. This libiary can be accessed by all of the users of Vision and therefore provides a mechanism for users to share process components.

\subsection{1; Prucess editor}

\subsubsection{1) Process and phase levels}

The process editor allows process models to be graphically constructed. Vision uses a two level system to represent process models. The first level is called the process level which is described in a process diagram. Each process model has one process diagram and it can contain start, end, synchro, dial, s, and phase objects (each of these will be further described later). The pracess level is used to represent the various phases in a process. Phases are defined as a set of related steps and therefore provide a level of abstraction because they allow a group of steps to be treated as a single object.

The second level is called the phase level. Each of the phases in the process level can be 'expanded' to a phase diagram. This diagram can contain start, end, synchro, dialog, generic, metric, and activity objects (again, each will be further described later). 
A given phase diagram gives the steps that make up an individual phase. Together, the two levels make up a proces!; model. A simple example of how Vision represents a process is shown in figure $! 1$.

\section{Phase Model}

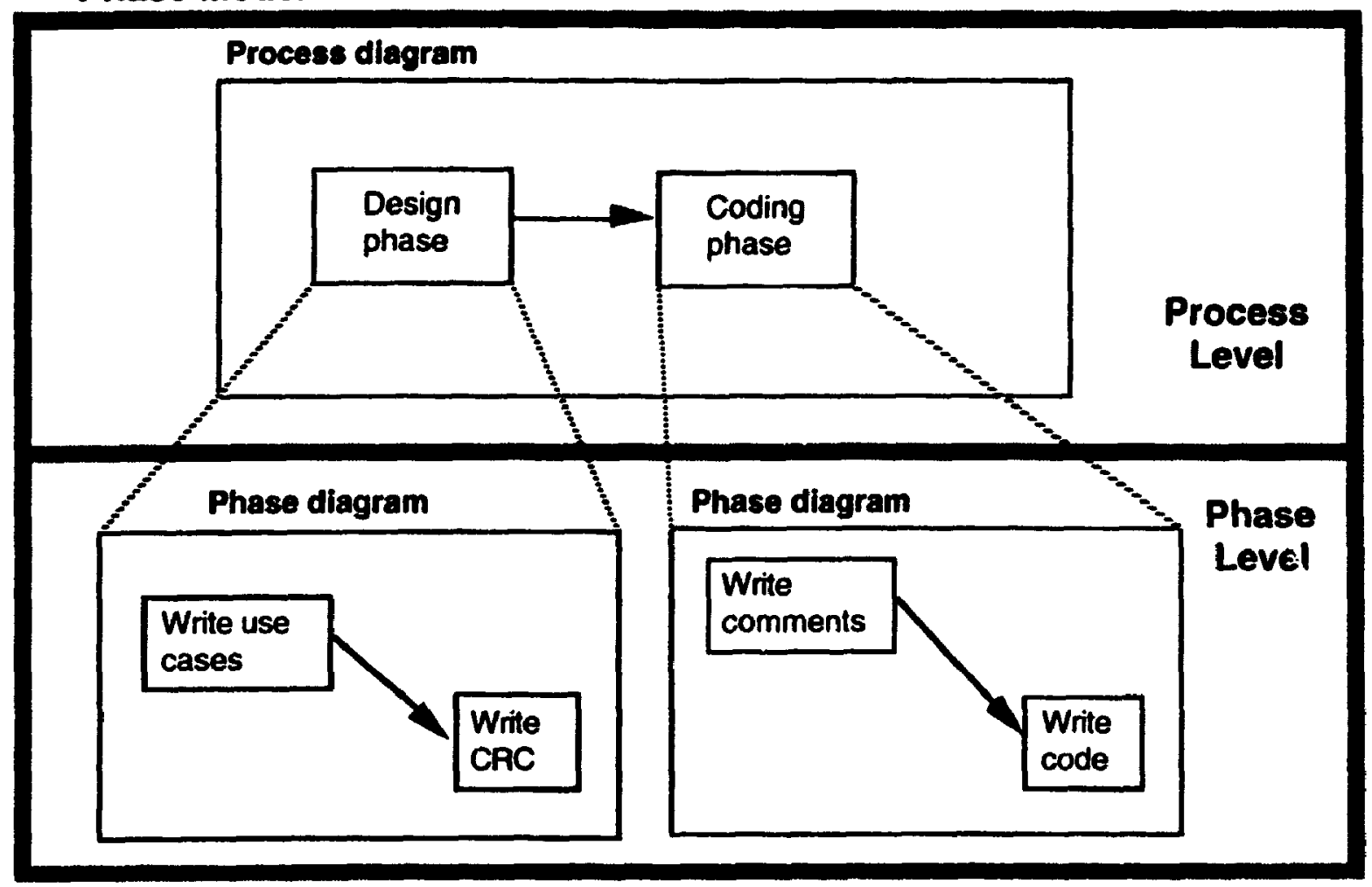

Figure 11: Process and phase levels

Figure 11 shows a process model that captures a design and implementation process. Note that figure 11 is not an actual view from Vision, it is just a conceptual representation of how process models, process diagrams, and phase diagrams are related. The process diagram contains the two phases (design and code) in the process. Each of the phases has a phase diagram stored with it. The phase diagrams contain the individual steps to perform to complete a phase. The directed links specify the control flow within a diagram. For example, in the design phase diagram, the 'write use cases' activity is 
linked to the 'write CRC' activity. This means that after the 'write use cases' activity is completed, the 'write CRC' is the next activity to perform.

\subsubsection{2) Process level objects}

As it was previously stated, a process diagram can have start, end, synchro, dialog, and phase objects. Each of these objects' function and enactment behaviour will be described below. A complete example of how an actual process is represented in Vision is shown in appendix 2. An example of enacting a process model is given in section 5.8 .

A process diagram must always contain a start and end object. The start object represents the starting point of the process. When it becomes active, it opens up a dialog asking the user to confirm that the process should be started. If the user does not confirm, nothing is done. If the user does confirm, the process object that the start model is linked to is activated. This represents the next process object to activate as specified by the control flow of the process diagram. The end object denotes the ending point of the process. When the end object becomes active, it means the process diagram has finished enactment. It opens up a dialog that tells the user the enactment of the process diagram has been completed and nothing further is done.

The phase object represents a phase in the process. The phase object has a name, checklist, and phase diagram associated with it. By using the editor, the user can configure each of these attributes. The name is used to identify the phase - e.g. testing phasc, coding phase, etc The phase diagram graphically represents the steps in the phase. By selecting a phase object in the editor, it can be expanded to show its phase diagram. This allows the phase diagram to be graphically edited. Once the editing is 
finished, the phase diagram can be collapsed to show the process diagram that it is contained in. The checklist stores items of interest or information relevant to the phase. When a phase object is activated, a window is opened that contains the checklist. This allows the developer to check off the items as they are completed. An example checklist for a coding phase is shown in figure 12. It shows that the developer has written the method comments and the test cases but still has to write the class comments. The state of the checklist is stored with the phase so that each time the checklist is opened, it remembers which items were checked and which items were not.

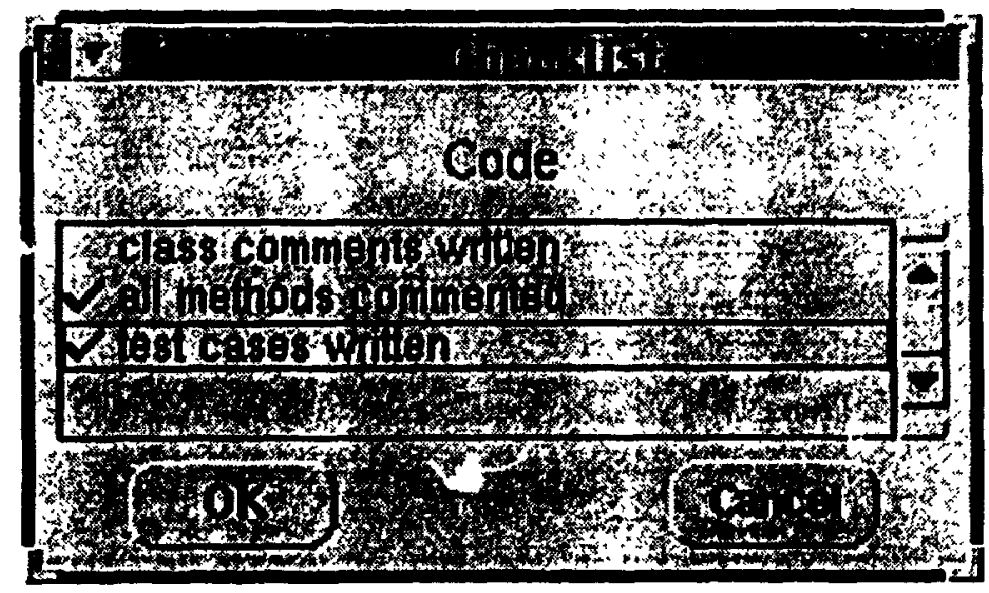

Figure 12: A checklist

The dialog object is used to ask a quesiion. Using the process editor, a question is stored with the dialog. When the dialog object is activated, a dialog window is opened that displays the question. The user can answer the question yes or no. This provides a true or false value on which a synchro object can branch.

The synchro object can have multiple incoming and multiple outgoing links. The synchro object is used to split one in-link into many out-links, merge many in-links into one out-link, or selectively branch based on a boolean argument. The editor is used to 
configure the synchro object's behaviour in the following manner. The synchro object can follow one of two behaviours when an in-link is activated. The synchro object can either become active when any one of its in-links are activated, or can only become active when a specified group of in-links have all been activated. Once the synchro object is active, it can either activate all out-links in a specified group of out-links or activate one group of out-links if it received a true argument ard another group of out-links if it received a false argument. These three types of sehaviours for the synchro object are shown in figure 13 .

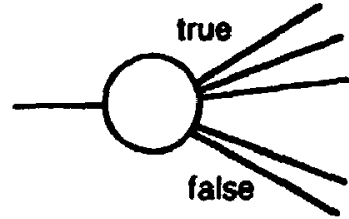

Branch

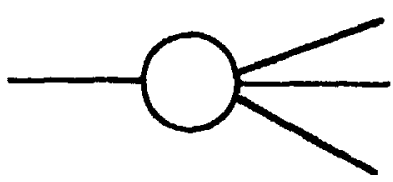

Split

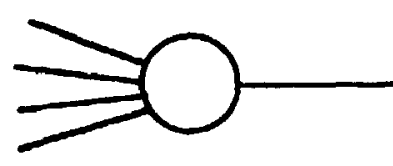

Merge

Figure 13: Synchro object behaviours

With the branch behaviour, the synchro object becomes active when its in-link becomes active and either activates the group of true or false out-links depending on the boolean argument. With the split behaviour, the object hecomes active when its in-link becomes active and activates the group of all its out-links. Finally, with the merge behaviour, the object becomes active when the group of all its in-links have been activated and then activates its out-link.

By linking each of these process diagram objects together, a process diagram is constructed. An example is shown in figure 14. The icons in the left side of the window will be explained in section 5.4.1.4. 


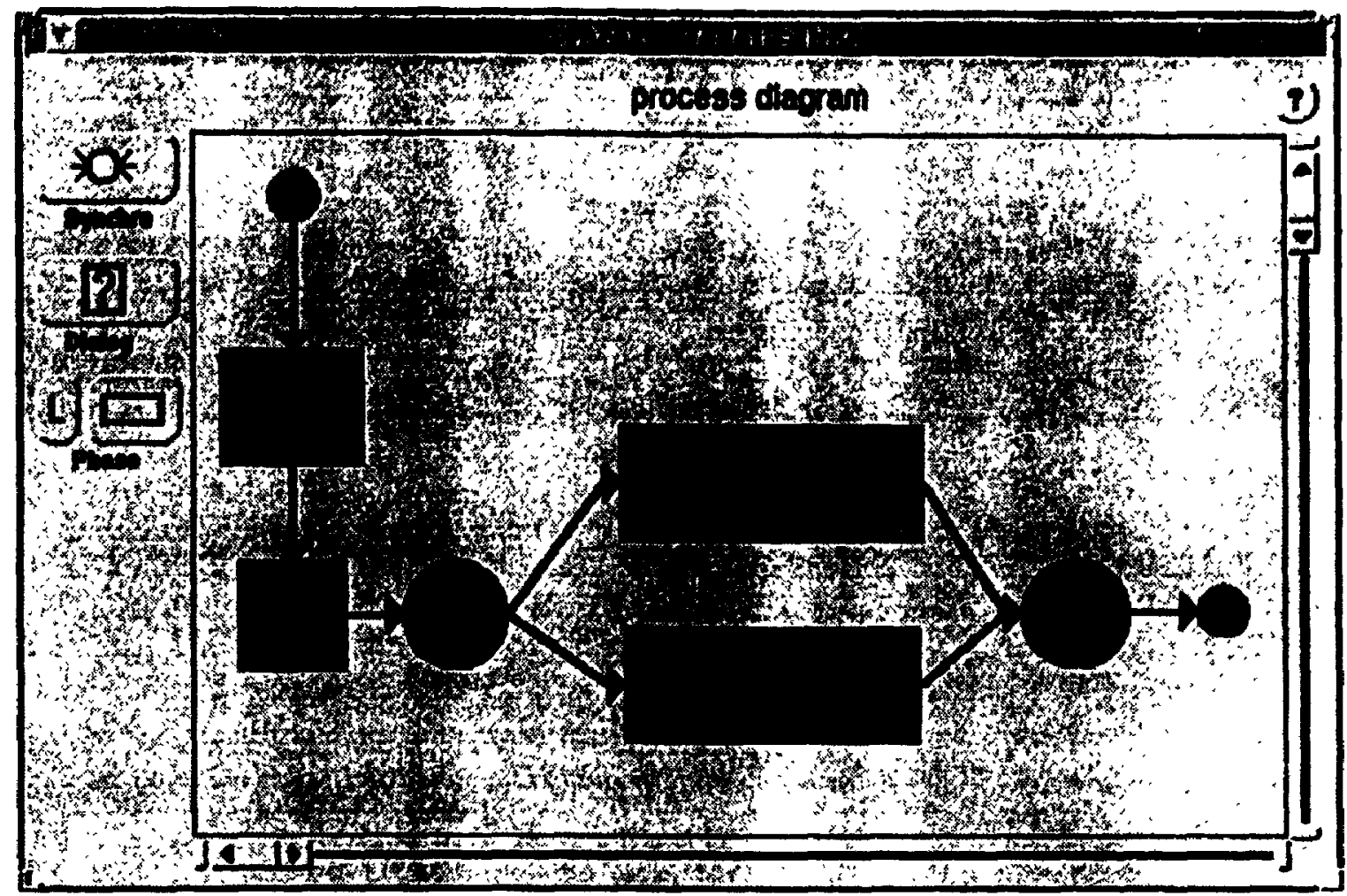

Figure 14: A process diagram

The process diagram in figure 14 represents an implementation and testing process. It has the following behaviour. After the process diagram is started, the code phase will be activated. This will enact the phase diagram that specifies how the coding phase is to be done. When the coding phase is completed, a dialog will ask the question 'Is the code a critical part of the system'. If the developer answers yes, the synchro object will branch to the high stress testing phase. Otherwise, the low stress testing phase will be activated. This allows the process to be guided by the product being developed. After testing, the process diagram will be done. 


\subsubsection{3) Phase level objects}

The phase level can have start, end, dialog, synchro, activity, metric, and generic objects. The start, end, dialog, and synchro objects have identical behaviour as described in the process level objects section. The function and enactment behaviour of the activity, metric, and generic objects are discussed below. Again, an example of enacting a process model is given in section 5.8 .

The activity object is used to represent activities or units of work in a phase. Activities have a name, information, and checklist associated with them. The editor is used to configure each of these parameters. The name provides a name for the activity. The information contains text describing how to perform the activity. Finally, the checklist provides relevant check items for the activity. It works in a similar fashion to the checklist feature in phase objects.

When an activity is activated, a control window is opened. This presents the information, checklist, and status buttons to the user. The information and checklist tell the user how to do the activity. The status buttons allow the developer to keep the tool up

to date as to their current status. An activity can either be current, on hold, or completed. If an activity is current, it means the developer is actively working on the activity. If an activity is on hold, it means the developer is still working on the activity, but at the current time is doing something else (e.g. they are in a meeting). Finally, if an activity is completed, it means the developer has finished the activity. It is through the control window that the developer controls the enactment of the process model. This is because the enactment of the model will not proceed until the developer has clicked the 'ok' button in the window. Through this mechanism, the developer makes Vision wait until they have completed the activity. An example control window is shown in figure 15. 


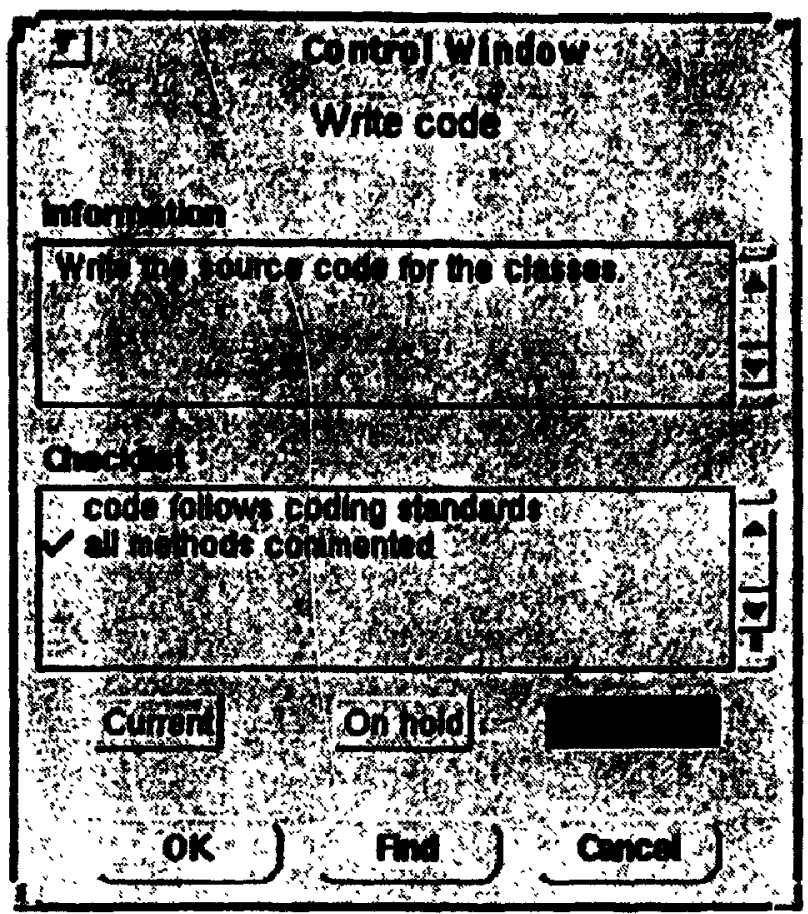

Figure 15: A control window

In the figure, the control window represents the 'write code' activity. The text in the information field tells the developer what to do in the activity. The checklist is used to check off items as they are done. The appropriate status button is selected by the user to represent their current status with respect to the activity. In figure 15 , the developer has indicated that they have completed the activity.

The metric object represents a goal, question, metric, and analysis (GQMA) object. GQMA objects will be descrilut in section 5.5. For the time being, GQMA objects can be viewed as metric collection points. When the metric object is activated, the metric associated with the GQMA object will be collected and stored.

The generic object allows Vision to work with other tools. The editor is used to associate a class name and class method name with the generic object. When the generic 
object is activated, the class method will be sent to the class. Depending on the class and method specified, this can cause arbitrary behaviour to be triggered. For example, this can be used to invoke an analysis tool or a tool that automatically generates test cases. In this manner, the process model provides the infrastructure to use many different CASE tools to give complete lifecycle coverage. The generic object was supported in Vision to make Vision as open and flexible as possible. However, working with other tools was not thoroughly pursued in this thesis and is left as an area of future research.

By using the process editor to link the phase diagram objects together, phase diagrams can be constructed. It is the phase diagrams that represent the steps for a given phase. Figure 16 shows an example phase diagram.

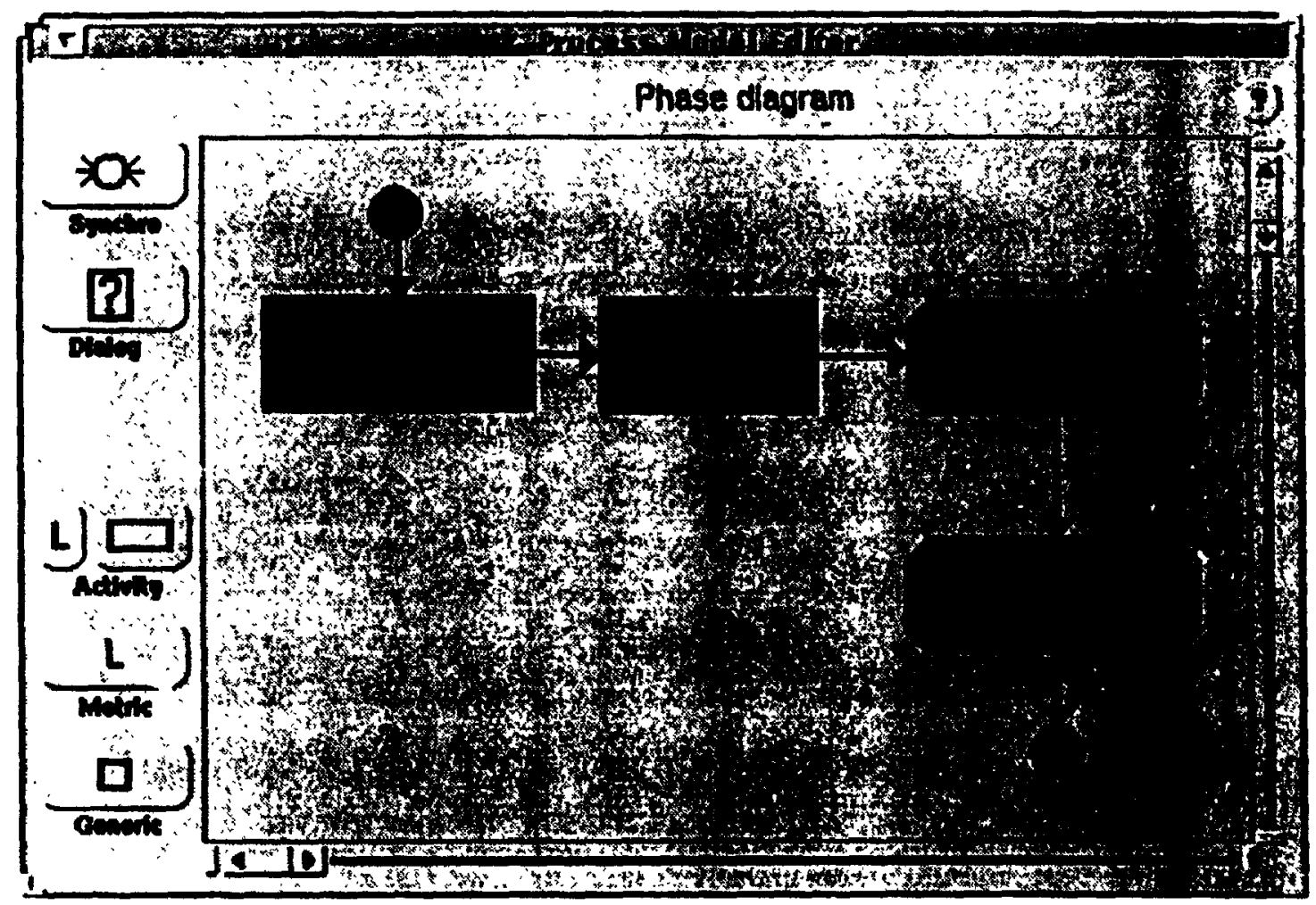

Figure 16: A phase diagram 
In this figure, a typical coding phase is described. The first two activities involve writing the comments and the code. After they are completed, a metric is run to collect the size of the code. Finally, a generic object is used to run a separate tool to generate test cases for the code that was written.

\subsubsection{4) Process editor features}

The previous sections described the objects that the process editor works with. The actual process editor window was shown in figures 14 and 16. The editor works in a similar fashion to any graphical editor. It allows the selection and multiple selection of the component objects. Menu items allow the objects to be linked, deleted, aligned, expanded, or collapsed. On the left side of the window, there are buttons that allow a particular object type to be added to the diagram. The buttons reflect which objects can be added to the current diagram, depending on whether a process or phase diagram is showing.

When a process diagram is showing, as in figure 14 , there are buttons to add synchro, dialog, and phase objects. Clicking on a button adds the appropriate process level object. to the center of the diagram. The button with an ' $L$ ' (for library) as its label that is next to the phase object button is used to access the process library. It allows the user to add a phase object that was previously stored in the library. This is an example of reusing process components to quickly construct new process models.

When a phase diagram is showing, as in figure 16 , there are buttons to add synchro, dialog, activity, metric, and generic objects. Again, clicking on a button adds the appropriate phase level object to the diagram. The two library buttons allow activity or metric objects to be retrieved from the library. Note that metric objects must be 
retrieved from the library. This is because there is a specialized browser to create metric objects which will be described in section 5.5.2.

Once a process diagram has been constructed, it can be saved to the process library (the library is described in the next section). Process diagrams can be retrieved from the library to be edited. Individual phase, activity, and metric objects can also be saved and retrieved from the library.

The last feature of the process editor is that it allows a given diagram to be enacted. This can be used to see how the diagram will behave when it is run. Enactment of a diagram occurs in a process model viewer window which is further described in the enactment section.

\subsection{2) Process browser}

The process browser is used to browse the contents of the process library. The process library contains process diagrams, phase objects, and activity objects that were created in the process editor and saved to the library. In keeping with the browser metaphor, the objects can be placed in different categories for added organization [Goldberg and Robson, 1983].

Within Vision, there are two process libraries - a local and global one. The local library is stored in a user's image at their machine. This : eeans that each user of the tool has their own local process library. This allows users to experiment and create various process models and store them locally. 
The global library is stored in ENVY's central repository. Vision only has one global process library that any user can access. This allows the users to import or export process components between the global and local libraries. Using this mechanism, the users can share process components amongst themselves. For example, a given user may create a new process model and store it in their local library. They can experiment and test the model to make sure it works properly. When the developer is satisfied with the process model, they can export it to the global library. The other developers can then import the model into their local library to be used as the new process model.

Having this separation between the local and global library is useful because the local libraries act as a private space for each developer. This means the global browser will only contain approved process components and won't be cluttered with components of questionable validity and usefulness. One member of the development team can be appointed as the global library supervisor. This person would be responsible for maintaining the global library and would control what is allowed to be placed into the library.

\subsubsection{1) Local process browser}

A picture of the local process browser window is shown in figure 17. Depending on the pull down menu, either process diagrams, phase objects, or activity objects are displayed. In the figure, process diagrams are shown. The names of the diagrams are grouped into categories. There are menu items to remove and rename category and object entries. Additionally, a category of objects or a single object can either be imported or exported to the global library. From the browser, it is also possible to open up the process editor on a diagram or enact a diagram in the process model viewer. 


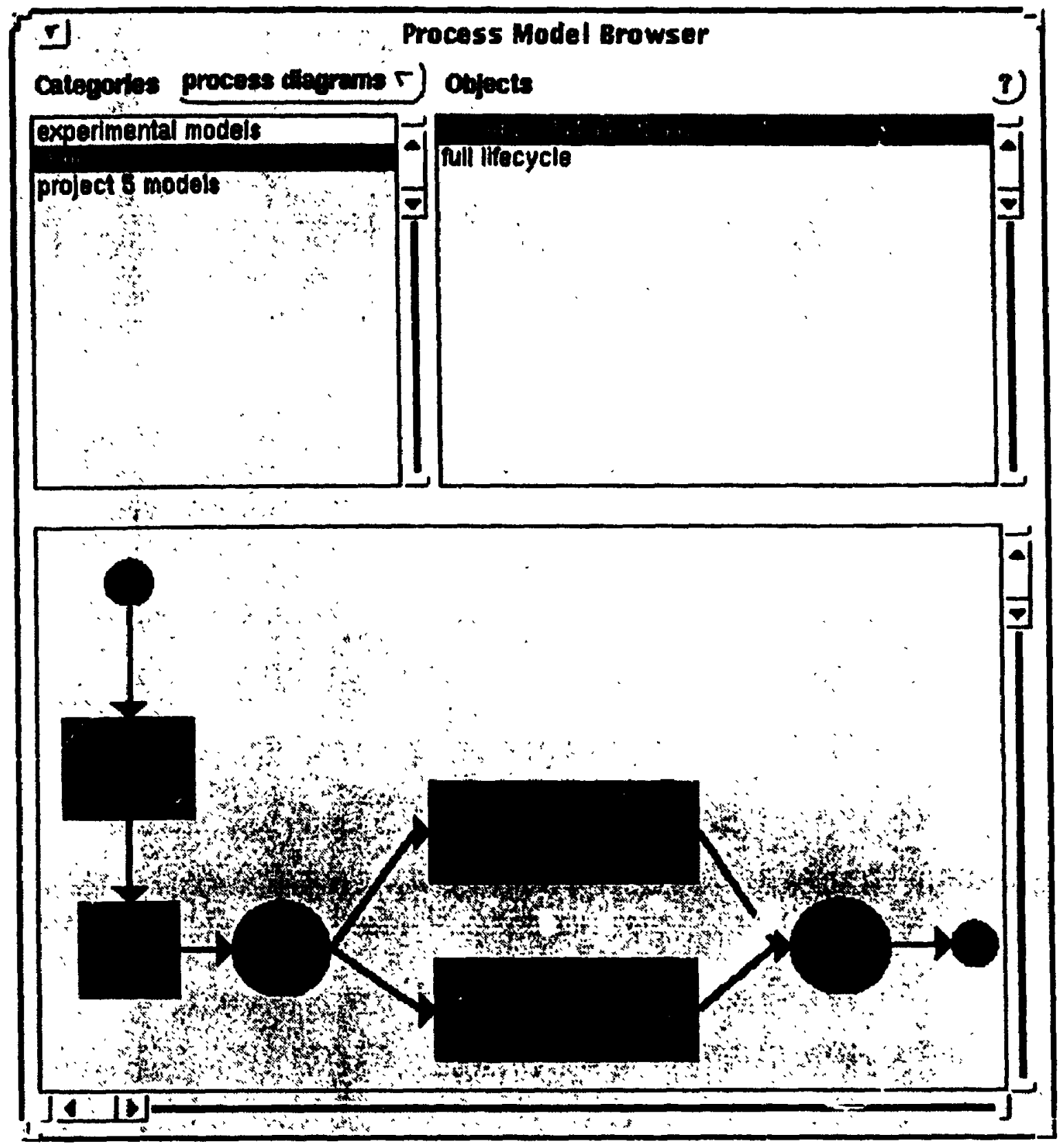

Figure 17: A local process browser 

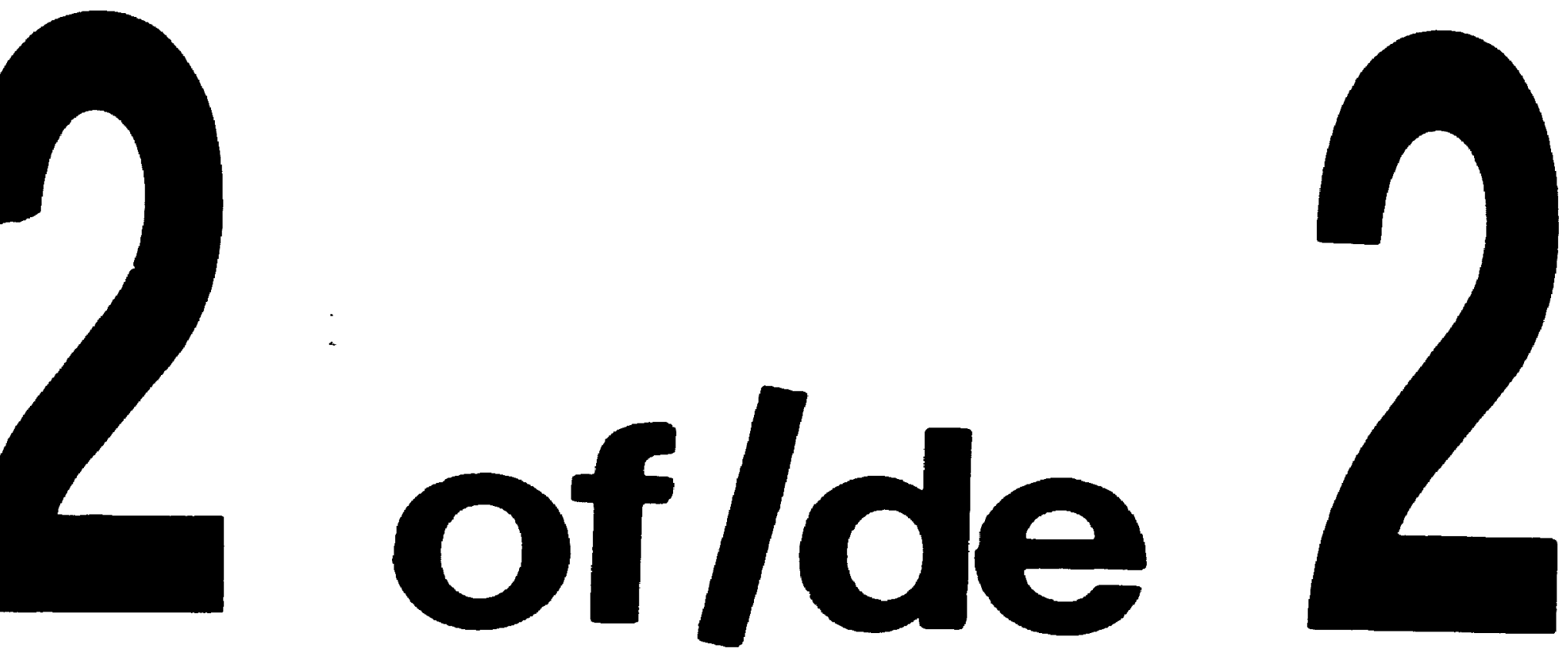

PM-1 3\%"x4" PHOTOGRAPHC MHCROCOPY TAREET was 1010 ansi/tso 22 EOUMALENT

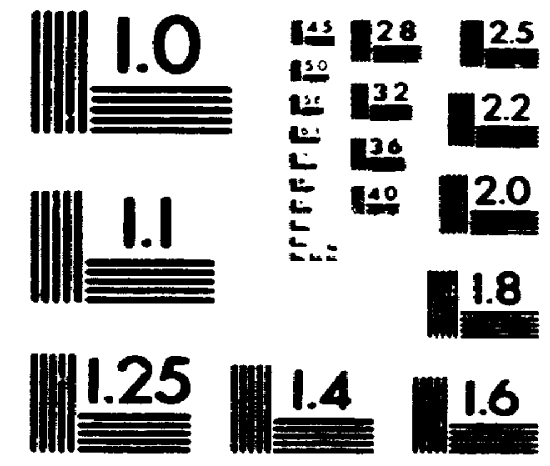

PAECISION"m RESOLUTION TARGETS 


\subsubsection{2) Global process browser}

The global process browser is nearly identical to the local process browser. The main difference is that it browses the global process library. When it is opened. the global browser is copied and stored locally. The global browser works on this local copy. After any changes are made, the copy is saved to the global library.

The global process browser is used to maintain the global process library. 'It allows a user to see what is in the library, to rename categories or objects, and to remove catcgories or objects.

\section{5) Metrics}

The metrics sub-system is made up of the local and global GQMA browsers. As shown in figure 10, the GQMA browsers are used to create the GQMA objects that are used in process models.

\subsection{1) GQMA objects}

In Vision, metrics are represented by a goal, question, metric, and analysis (GQMA). This builds on work by Basili and Romhach in which they represented metrics with a goal, question, and metric [Basili and Rombach, 1988]. Basili and Rombach found that by associating a goal and question with a metric, it was easier to see what the metric was used for in a project. The natural evolution of this idea is to additionally associate analysis techniques with the goal, question, and metric. Therefore, not only will 
the metric's context be known, the analysis to perform on the metric will also be known. An example of how a GQMA object is represented in Vision is given below.

Goal: A sentence stating the goal of the metric.

e.g. "Make sure the testing of a class is adequate"

Question: A sentence giving a question that quantifies the goal.

e.g. "What is the percent test coverage for the class"

Metric: The metric or metrics to collect to answer the question.

e.g. Dynamically measure the number of methods in the class that were called during the execution of a test suite for the class. Make this a percentage by dividing by the total number of methods in the class.

Analysis: A description of the way in which the data is used to answer the question, address the goal, and benefit the project.

e.g. Compare the test coverage for a class with a pre-set target. If it is below the target, improve the test suite. This will ensure a minimal level of testing for all classes.

e.g. View a histogram giving the distribution of test coverage for all classes. Find the classes with the lowest test coverage values and look to do more testing on these classes.

As the example shows, by viewing the metric in the context of the goal and question, it is clear what the metric's purpose is. Furthermore, the analysis gives 
instructions on how to analyze the data. This allows anybody, not just the person who created the metric, to use its results to benefit the project.

In Vision, GQMA objects are placed in process models. This a!lows them to be enacted like any other process model object (the enactment behaviour of GQMA objects is discussed in section 5.8.2). In this manner, metric collection is integrated with the process. Since the process is followed throughout the project, the metrics will be consistently collected in the project. This allows metries to be compared across the project and even across different projects.

\subsection{2) GQMA browsers}

The GQMA browsers are used to create GQMA objects and view the GQMA objects stored in the library. Just as with the process libraries, there are local and global GQMA libraries. This allows individual users to create and experiment with GQMA objects in their local library and share useful ones with each other through the global library.

A picture of the local GQMA browser is shown in figure 18. In the top left field, the various categories of GQMA objects are listed. This field's menu has items to create, remove, and rename categories. By selecting a particular category, the goals associated with the category are displayed. These are listed in the top right field. This field has menu items to create, remove, and rename goals. By selecting a goal, the questions associated with the goal are given. The questions are listed in the middle right field. This field's menu has items to create, remove, and rename questions. Finally, by selecting a question, the metric and analysis information is placed in the bottom field. Within the metric and analysis part of the browser, the user enters the following information. A 
metric name gives the name of the metric. This allows the metric to be identified. The metric class name and metric method name provide the class and method to call in order to collect the metric. Finally, a description of the analysis technique is entered into the analysis field. This instructs the user on how the metric was meant to be analyzed in order to answer the question and address the goal. In figure 18, the test coverage GQMA is shown.

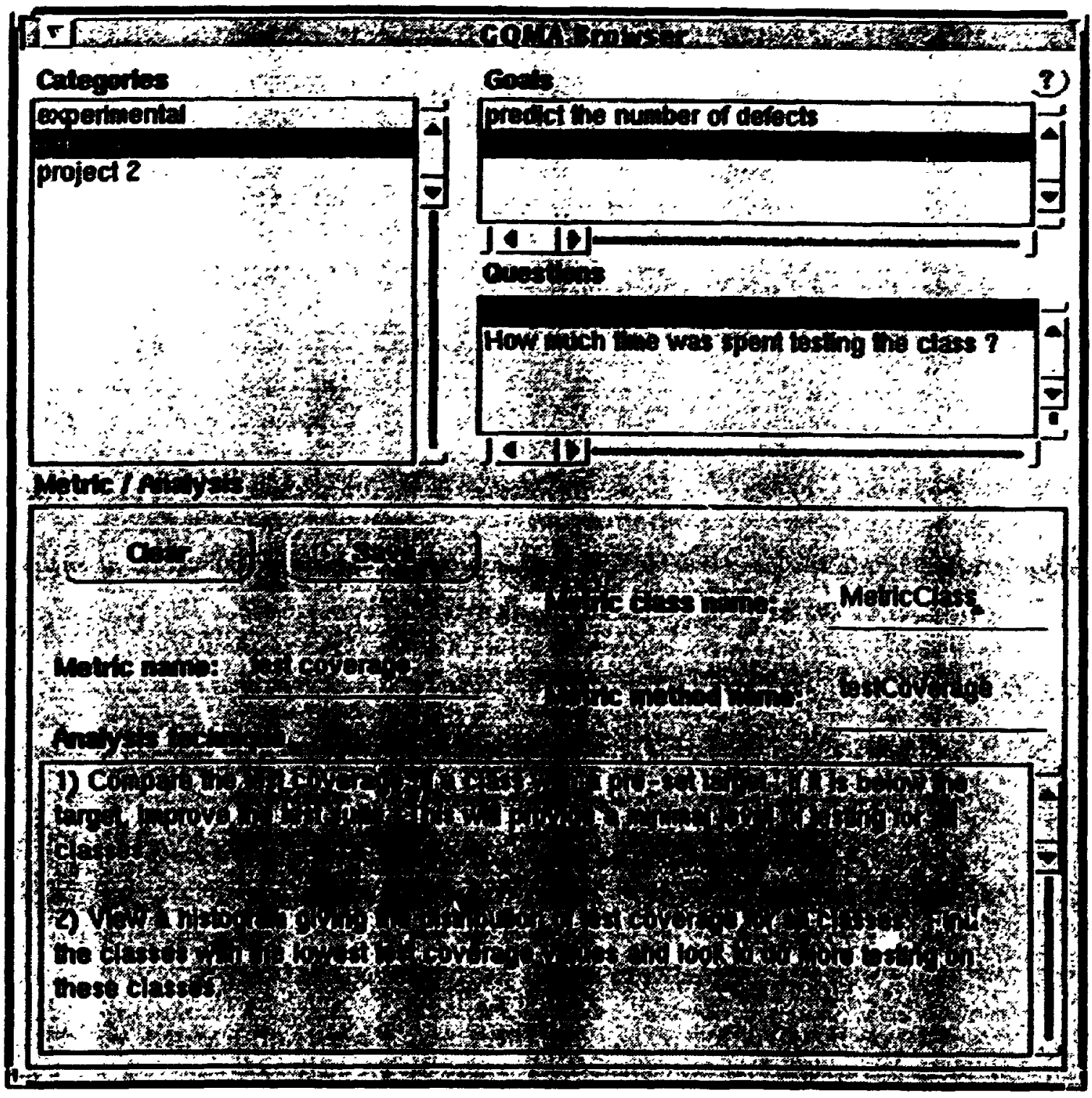

Figure 18: A local GQMA browser 
By using the local GQMA browser to specify a new goal, question, metric information, and analysis information, a new GQMA object is added to Vision. In addition to this, the metric method that actually collects the metric must be implemented. This is done in the normal way that methods are added to Smalltalk. Vision has a metric class that contains much of the framework for collecting metrics. The new metric method just has to add its specific functionality to the existing framework.

Similar to the local process browser. GQMA objects can be imported or exported to the global GQMA library. The global GQMA library is viewed using the global GQMA browser. This browser is basically identical in appearance and function to the local GQMA browser. The only difference is that it does not allow GQMA objects to be created. The global GQMA browser works in the same way that the global process browser does - the global library is copied locally, changes are made to the copy, and the copy is saved back to the global library.

\subsection{3) GQMA objects proposed}

The main purpose of this thesis was to research and develop the framework of Vision. The specific process models and metrics to use in Vision came from other people's research that was described in the section on relevant work. With respect to metrics, eight GQMA objects are proposed. These GQMA objects use metrics that were discussed in the section on object oriented metrics. Since the field of object oriented metrics is so new, there is no consensus on which object oriented metrics provide the most benefit to a project. Therefore, the metrics that were selected came down to a personal judgment as to how useful they would be and how easily they could be 
automatically collected. These eight GQMA objects provide the initial metrics which Vision supports. As Vision is used over different projects and more metrics are required. its library of metrics can be augmented accordingly. The eight GQMA objects are described below.

\subsubsection{1) GQMA 1}

G) Make sure that testing is adequate.

Q) What is the percentage test coverage.

M) Measure the percentage test coverage for a class (the number of methods in the class that were called by a test suite divided by the total number of methods in the class).

A)

1) Compare the percent test coverage of a class to a target value. If it is below the target, improve the test suite.

2) Compare the percent test coverage for all classes. Look to improve the test suites of classes with low values.

This GQMA builds on research in measuring test coverage for structured languages. For structured languages, test coverage is usually expressed as the percentage of source code lines that were executed [Conte et al., 1986]. Applying this to object oriented languages, methods are chosen as the base unit of measure. This is because it makes more sense to find methods that were not tested than it does single lines of code. This metric is similar to Haaland's runtime method coverage metric [Haaland, 1992]. 


\subsubsection{2) GQMA 2}

G) Increase quality by improving defect prone code.

Q) Which parts of the code are prone to change.

M) Collect the number of times an application, class, and method is changed.

A)

1) Compare the metric value for an application, class or method to a project average.

If it is above average, look to clean up the class or method since the changes may have introduced errors.

2) View the metric values for all classes. Find the classes with the highest values. Look to clean them up or do regression testing to make sure the changes have not introduced defects.

This GQMA also builds on research done with structured metrics. When portions of code undergo many changes, there is a high probability that errors have been introduced [deMarco, 1982]. This is applied to object oriented languages by measuring the changes made to the language's organizational constructs (i.e. methods, classes, and applications ${ }^{7}$ ). In this manner, defects can be localized to code. This type of metric was proposed by Haaland as his evolutionary change metric [Haaland, 1992].

\footnotetext{
7 The concept of applications is provided by lENVY, a configuration management system, and not by an object oriented language. However. applications form an integral part of the language when ENVY is used so they are considered a language construct.
} 


\subsubsection{3) GQMA 3}

G) Increase quality by improving defect prone code.

Q) Which parts of the code undergo many reviews and re-work.

M) Collect the number of reviews before success for an application or class.

A) Perform the same analysis as for GQMA 2.

This GQMA follows he same line of thought as for GQMA 2. Frequent code reviews mean there were problems with the code that had to be fixed. This makes the code more likely to contain errors that may have been inadvertently introduced with the code fixes. There is a metric similar to this one for structured languages [deMarco 1982].

\subsubsection{4) GQMA 4}

G) Increase quality by improving defect prone code.

Q) Which parts of the code have a high proportion of defects.

M) Compute the defect density for a class by counting the number of defects and dividing by the class' size.

A)

1) Compare the defect density for a class with an average value.

2) View the defect densities for all classes and pick the classes with the highest values.

3) View a histogram showing each class and the percentage of total defects it accounts for.

For each of the above, look to re-write classes with high defect counts. 
From research in structured metrics, defect density is defined as the number of defects per thousand lines of code [Conte et al. 1986]. To apply this metric to object oriented languages, defects should be related to $c$ ass size. This allows defects to be attributed to the object that contains the defect.

\subsubsection{5) GQMA 5}

G) Tailor testing and maintenance to characteristics of the code.

Q) Is the class strongly coupled to others by messages.

M) Dynamically measure the number of methods called or number of classes that are used when a test suite is run. Statically count the number of methods sent from a class.

A)

1) Compare the values for a class to an average value.

2) Look at all the classes' values and find the classes with the highest values.

For both of the above, classes with large values will be strongly coupled by message passing. This means that a class will be harder to test in isolation. In order to verify the class, it must be tested with the other classes it collaborates with. The class will also be harder to understand and motify in isolation. The public interfaces of the other classes must be understood to see how the class works. Furthermore if, the class' public interface is changed, the other classes will have to be re-tested.

This metric came from Chidamber and Kemerer's coupling between objects metric and $\mathrm{Li}$ and Henry's message passing coupling metric [Chidamber and Kemerer, 1991] and [Li and Henry, 1993]. 


\subsubsection{6) GQMA 6}

G) Tailor testing and maintenance to characteristics of the code.

Q) Is the class strongly coupled by inheritance.

M) Collect the number of superclasses, number of inherited attributes, and number of inherited methods.

A)

1) Compare the values for a class to an average value.

2) Look at all the classes' values and find the classes with the highest values.

For both of the above, large values indicate strong inheritance coupling. This means the testing of a class will be more dependent on testing the superclass(es) since the class uses much of the superclass(es) state and behaviour. For the same reason, it will also be harder to understand and modify the class without additionally understanding the superclass(es).

This metric applies Chidamber and Kemerer's depth of inhcritance tree metric and Chen and Lu's class hierarchy metric [Chidamiber and Kemerer, 1991] and [Chen and Lu, 1993]. 


\subsubsection{7) GQMA 7}

G) Tailor testing and maintenance to characteristics of the code.

Q) Does the class have many dependent children.

M) Collect the number of children, number of methods that will be inherited and not overridden, and number of inherited attributes.

A)

1) Compare the values for a class to an average value.

2) Look at all the classes' values and find the classes with the highest values.

For both of the above, large values indicate strong children dependency. This means that it will be harder to modify the class without affecting the children. If the class is modified, the children will have to be re-tested.

This metric uses Chidamber and Kemerer's number of children metric and Haaland's class level reuse metrics [Chidamber and Kemerer, 1991] and [Haaland, 1992].

\subsubsection{8) GQMA 8}

G) Increase the reusability of classes.

Q) Which classes need generalization.

M) Collect the method size of each method in a class and the number of methods in a class.

A)

1) Look at the method sizes for each method in a class and find methods with large sizes. 
2) Compare the average method size for all classes and find the classes with large average method size.

For both of the above, large values indicate large method sizes. Look to make large methods smaller by distributing the method functionality into two or more methods. This increases reuse and makes the class more general.

This metric applies Haaland's method length and class size metrics [Haaland, 1992].

\section{6) Management}

The management sub-system is mar : up of the managers interface. As shown in figure 10, the managers interface is used to create and view the work packages that define the tasks that developers are to complete.

\subsection{1) Work packages and projects}

As it was previously discussed, the work on a software project can be broken down into many tasks. It is at the task level that projects are tracked so it is the tasks that form the base unit of measure in the project plan. A project has many different types of tasks associated with it. Some examples include developing features of the system, participating in training classes, or attending weekly meetings. In the context of Vision, tasks are constrained to be tasks representing work to develop the system. This is because these tasks represent the bulk of the work of the project and would benefit the most from additional tracking. 
One of Vision's objectives was to provide a finer level of tracking tasks. This is done through process models. Since the lasks are all performed using the same software development process, a process model can be used to track the individual steps that occur in a task. Therefore, in order for Vision to use its process modeling capabilities to track tasks, it must somehow represent tasks. This is done using work packages.

A work package represents a unit of work in Vision. It will usually be directly mapped to a task in the project plan. This means that for every task in the project plan, there will be a corresponding work package in Vision. Note that this one-to-one mapping need not always hold. If a task in the project plan is large, it may be represented by many work packages. Conversely, several small tasks in the project plan may be represented by one work package. In any case, work packages allow Vision to be related to the project plan.

Work packages store the following information. The name gives the name of the work package. This gives a means of identifying the work package. The activity number associates a number with the work package. This gives a further mechanism to identify the work package. It can also be used to associate the work package with other project information. For example, if the organization uses numbers to identify bug reports, a work package tracking the fix of a particular bug can be assigned the bug's identifying number. This links the work package with the bug report.

The status represents the priority of the work package. This provides an indication of how important the work package is (e.g. urgent, medium, normal, low). The percentage complete contains an estimate of how much of the work package has been completed. It allows the developer who is responsible for the work package to 
communicate their progress. The relevant applications list stores the names of the ENVY applications that are affected by the work package. It is these applications that contain the classes and methods that implement the work specified by the work package. This allows tasks to be tracked to the source code. The relevant PERT nodes list contains the names of the tasks (nodes) in the project plan (PERT chart) that track the work package. This is how Vision and separate project management software can relate to each other.

The list of process models gives the process models that specify how the work package is to be completed. By following these models and keeping them up to date with the current status, the work package is tracked in detail. The start date and end date contain the dates when the work package was started and completed. This information is calculated from the process models associated with the work package. By parsing the process models and determining when the start and end objects in the process diagrams were activated, the dates when the work package was started and finished can be concluded. Finally, the developers list contains the names of the developer or developers who are assigned to the work package. Exactly one of these developers (in the case when there is more than one developer working on the package) is made the primary developer. Only the primary uuvelo- ar has write permission on the work package. Therefore, this developer is responsible for updating the percentage complete and status of the process models.

In Vision, work packages are stored in project objects. A project contains a name and a list of developers working on the project. Projects offer a logical means by which to group work packages. Furthermore, within a project, work packages can be stored in different categories. Categories can be used to group together all work packages associated with a particular development iteration. This is depicted in figure 19. 


\section{Project: Develop a simple calculator}

Development iteration 1

Development iteration 2

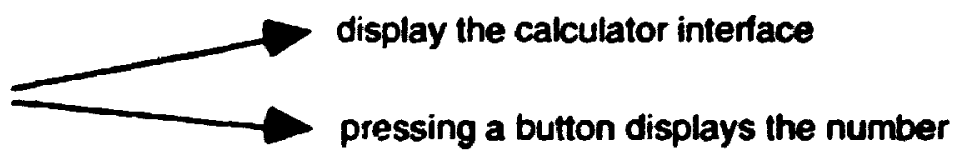

support arithmetic operations

pressing $=$ displays the result

Figure 19: The relationship between projects, categories, and work packages

In the firure, the project involves developing a simple calculator application. It contains two development iterations. In the first iteration, the calculator is graphically displayed and records which of its buttons were pressed. These tasks were tracked using two work packages. In the second itcration, the calculator computes and displays the results. These tasks were also tracked with two work packages. This example illustrates how Vision represents an iterative development effort by using projects, categories, and work packages.

\subsection{2) Managers interface}

Given the two object types, work packages and projects, it is obvious that Vision has to provide a mechanism to create and view these objects. This is done through the managers and developers interfaces. The managers interface, which is discussed in this section, allows the project manager to create projects and work packages and assign them to developers. The developers interface, which will be di icussed in section 5.7, allows the developers to view their assigned work packages. 
A picture of the managers interface is shown in figure 20 . It shows the work packages in the selected category and project.

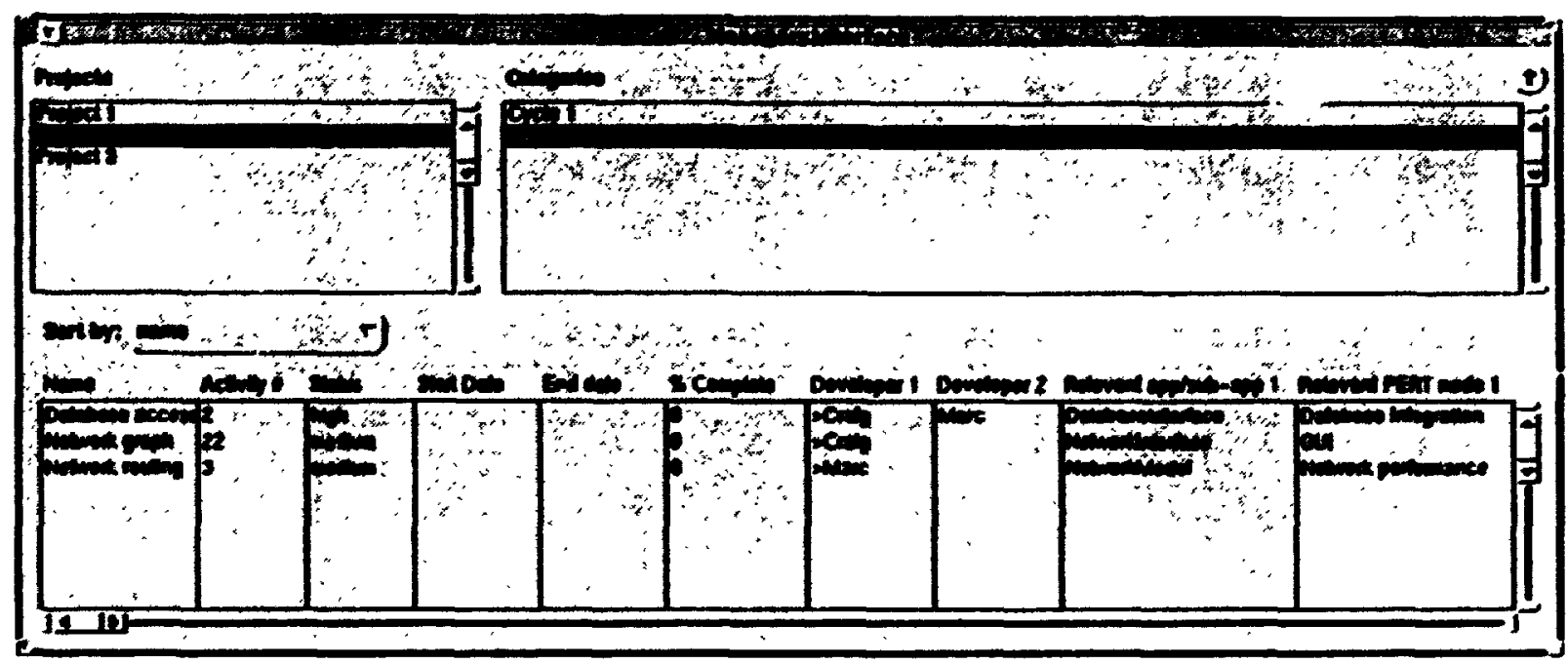

Figure 20: A managers interface

The upper left field contains the list of projects. The field's menu has items to create, edit, and remove projects. Projects have a name, a list of developers on the project, and a text field containing information on the project. This is shown in a dialog window that describes the project. The window is opened by selecting a project and choosing the edit menu item.

When a project name is selected, the categories in the project are displayed in the upper right field. In the figure, 'Project 2' is selected and the categont.s called 'Cycle 1' and 'Cycle 2' are displayed. Categories have a name and text information associated with them. The category field has menu items to create and remove categories and to edit and view the selected category's information. 
When a category is selected, all of the work packages that are stored in the category are listed in the bottom table. In figure 20 , the three work packages associated with the 'Cycle 2' category are shown. Each of the table's columns contains an attribute of the work package. The purpose of each attribute was explained in section 5.6. The pop up menu above the table allows the work packages to be sorted by different criteria. In the figure, the work packages are sorted by their name. They could also be sorted by developer, activity number, status, start date, end date, percent complete. developers, relevant applications, or relevant PERT nodes (project plan tasks).

When work packages are created, a dialog is used to enter all of the work package's information. For a given work package in the table, there are menu items to remove, edit, or view the process models associated with the work package. By viewing the process model of a work package, the manager can get an immediate indication as to which step of the process the developer is working on. This is done through a process model viewer and is shown in figure 22 in section 5.8.1.

The managers interface can print the entire list of work packages in the table to the Smalltalk transcript (the Smalltalk transcript is a window where information can be displayed in the Smalltalk environment). This allows the information to be copied and placed in a document - like a weekly status report on the project's tasks.

\section{7) Developer}

The development sub-system is made up of the developers interface. As shown in figure 10, the developers interface is used to view the work packages that are assigned to a particular developer. 


\subsection{1) Developers interface}

A picture of the developer's interface is shown in figure 21 . It displays all of the work packages assigned to the selected developer in the selected category and project.

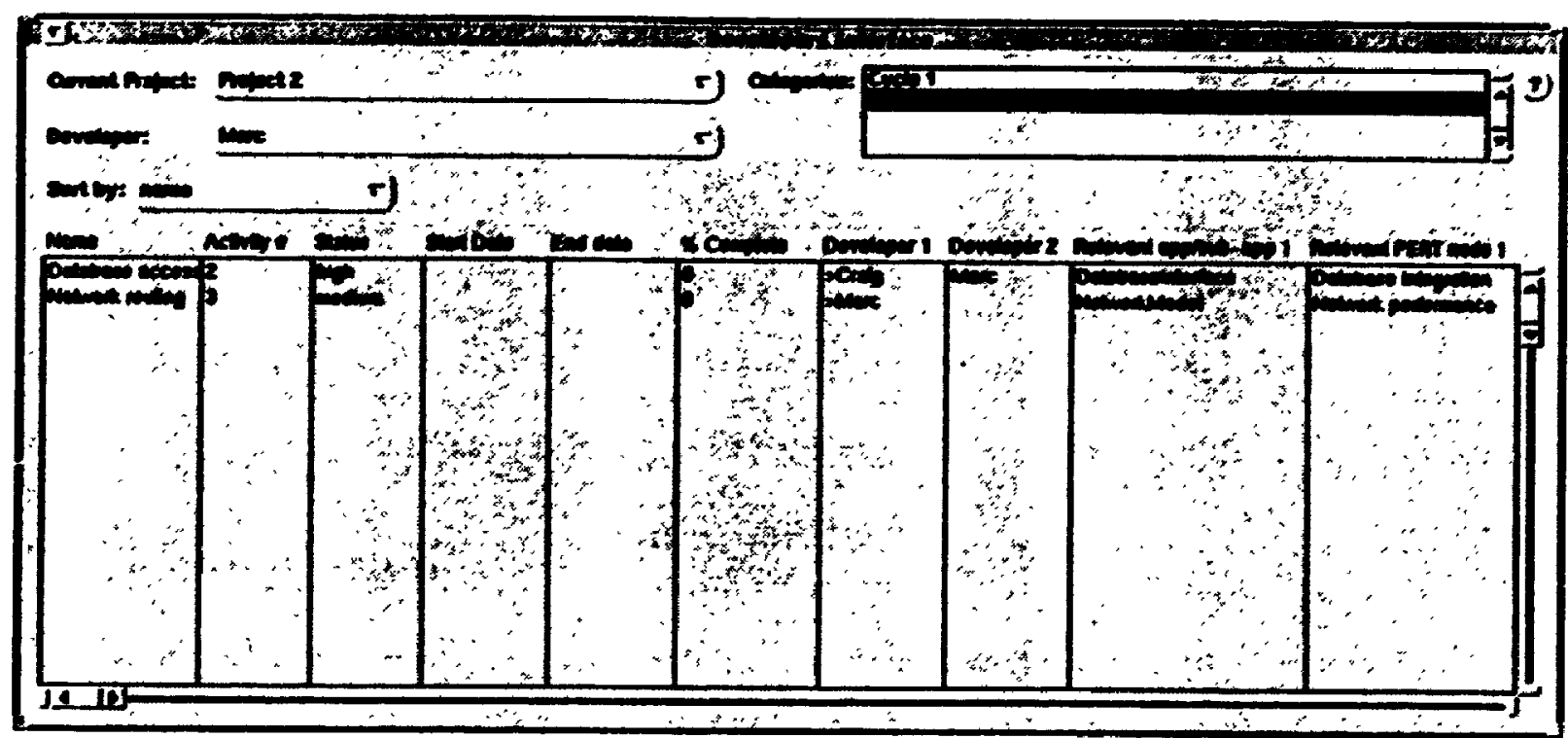

Figure 21: A developers interface

The developers interface works on the same general principles as does the managers interface. The work packages for the selected project and cycle are displayed in the table. In figure 21, the work packages for the project named 'Project 2' and category named 'Cycle 2' are displayed. The only difference with the developers interface is that it only displays the work packages assigned to the selected developer. This allows the developer to concentrate solely on their relevant work packages. In figure 21, the two work packages that are assigned to the developer named 'Marc' are displayed. The ' $>$ ' in front of a developer's name indicates that the developer is the 
primary developer. In this figure, the selected developer is the primary developer for only one of the work packages.

The operations that can be performed in the developers interface are limited. Creating, renaming, or removing projects, categories, and work packages is not allowed. This is because the distribution of tasks should be up to the manager. The developer should only be responsible for doing the tasks. To help with this, the developers interface allows the information for a category or work package to be viewed and edited. Additionally, the percentage complete attribute for a work package can be modified. Most importantly, the process models associated with a work package can be displayed and enacted. This provides guidance to the developer on how to do the work package. Furthermore, by following the process and keeping the model up to date with their current status, it allows others to see which step the developer is at in the process. The enactment of process models is illustrated in the next section.

\section{8) Enactment}

The enactment sub-system is made up of the process model viewer, metrics analysis interface, and metrics results window. As shown in figure 10, the process model viewer allows the developers to follow the process and lets managers view their progress. From the process model, any metrics that are collected are analyzed with the metrics analysis windows. In this section, all views depicted represent the views that developers will see. 


\subsection{1) Process model viewer}

A picture of the process model viewer is shown in figure 22 .

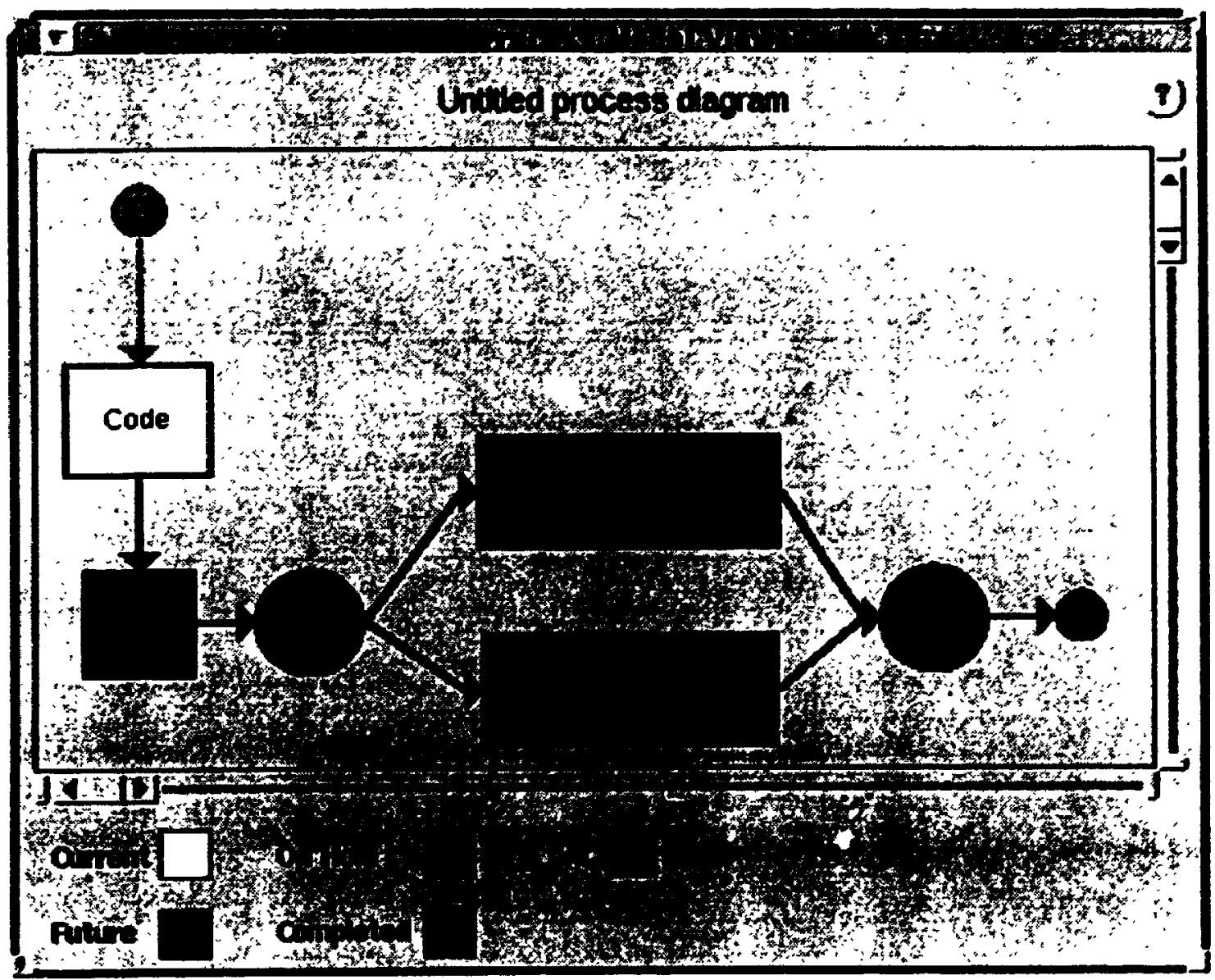

Figure 22: A process model viewer

In the figure, each object in the process model is coloured according to its status. The status indicates if the object has been completed, is currently being performed, is on hold, is not applicable, or will be done in the future. Colour patches at the bottom of the window match colours to status. This provides an easy mechanism to determine which step the developer is currently working on in the process. For example, in figure 22 it can be quickly determined that the coding phase is the one currently being performed. The 
When the process model viewer is initially opened, all of the objects have a status of future. This means that the diagram has not been enacted. To start the enactment, the start menu item is invoked. This creates a message send to the start object in the process diagram. Upon receiving this message, the start object sets its status to current and opens up a dialog asking if the user wants to start the enactment. If the user confirms, another message is generated and is sent to the object that the start object is linked to. In figure 22 , this object is the coding phase. As it was previously described, when a phase object is activated, it displays its checklist and starts the enactment of its phase diagram. This is shown in figure 23 .

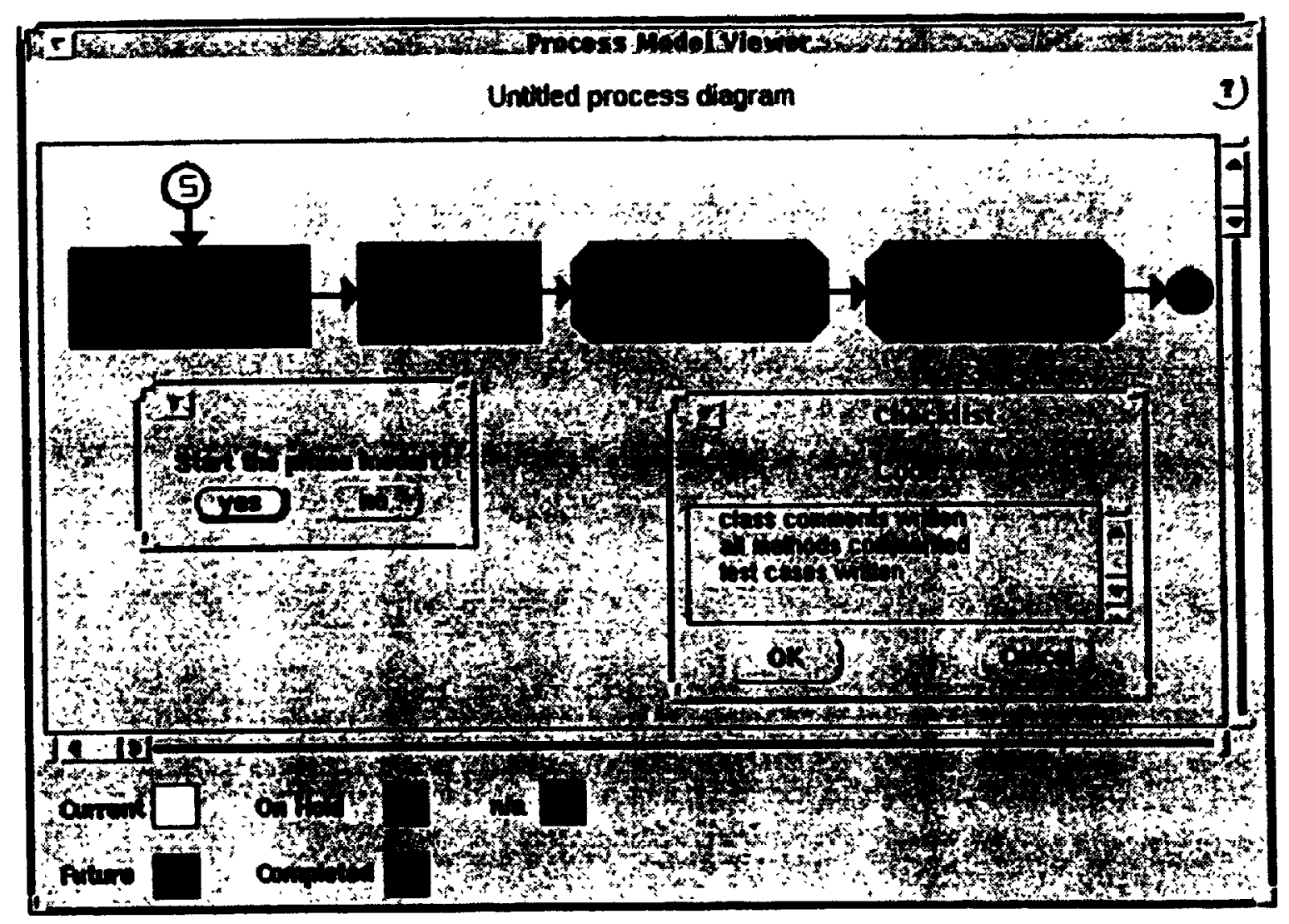

Figure 23: The enactment of a phase diagram

In the figure, a dialog asks if the user wants to start the enactment. If the user confirms, the object that the start object is linked to will be activated. In this case this 
object is the 'Write comments' activity. When an activity in the phase diagram is started, the activity displays its control window. This tells the developer what to do and allows the developer to give their status to the tool. The activation of the coding activity is shown in figure 24.

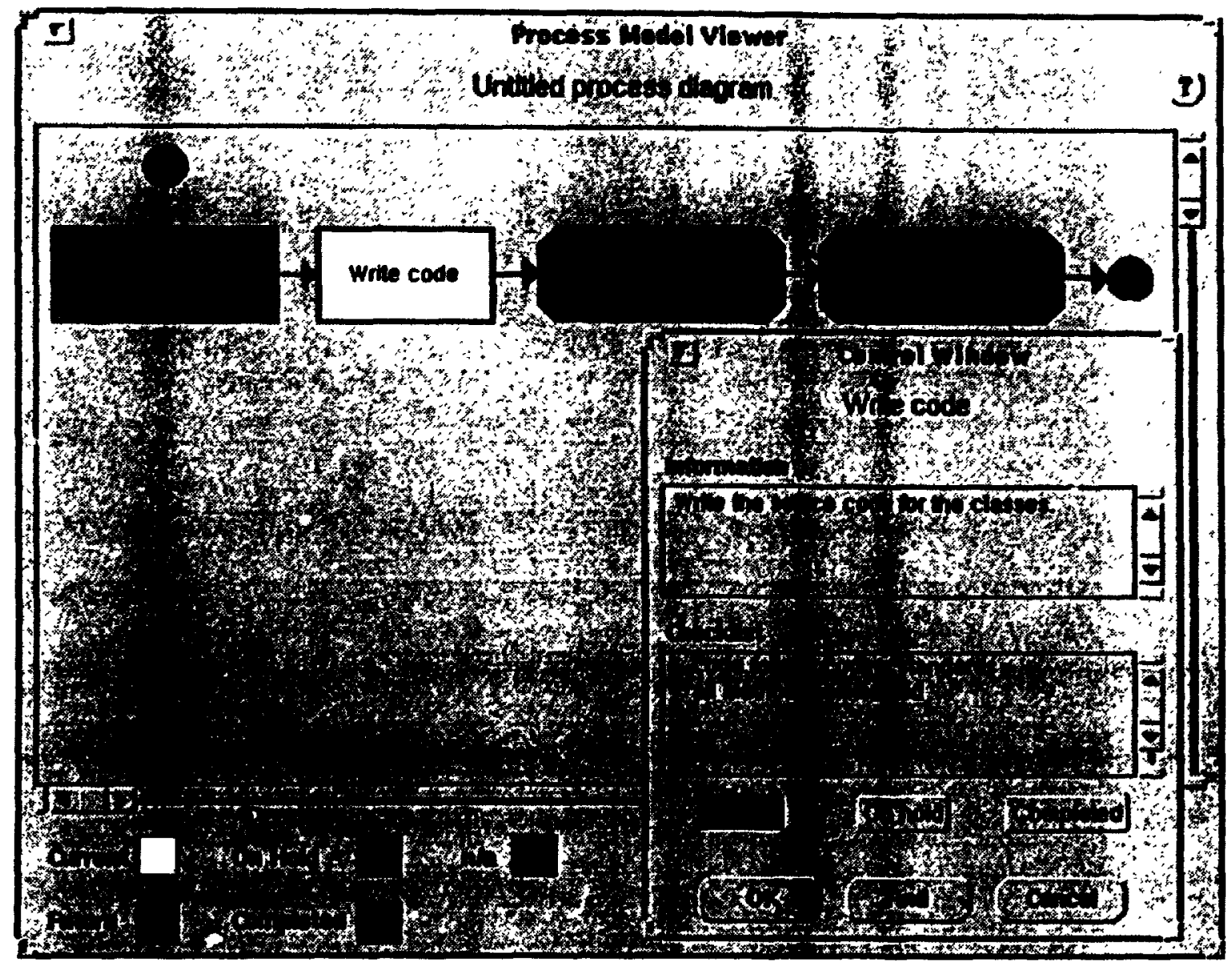

Figure 24: The enactment of a coding activity

When an activity object is the current object that is activated, the enactment is suspended. The tool will wait until the user makes a status change (by clicking an appropriate status button in the control window) and closes the control window (by clicking the ok button). Once this is done, it is taken to mean that the user has finished the activity and is ready to move on to the next step in the process. 
The enactment of the coding phase diagram continues until the end object is activated. This signals the end of enactment for the phase diagram. The process diagram is brought into view and the object that the code phase is linked to is activated. This scenario of activating the linked objects by messages continues until the end object in the process diagram is activated. When this happens, a dialog is opened that tells the user that the enactment has finished. No more messages are generated so the enactment stops.

As it was previously discussed, by viewing the process model during the enactment, a developer's progress in the model can be determined. After a process model has finished enactment, a summary of the enactment can be generated. This is shown in figure 25 .

During the enactment, the time when the various transitions occurred was automatically recorded by Vision. The enactment summary presents the status transitions of the process model objects in the form of a hierarchical list. This allows managers to track the time that was spent in each phase. For example, in figure 25 , it can be determined when the phase object named 'Code' was started (its status changed from future to current) and when it was finished (its status changed from current to completed). Indented underneath the phase object's information is the status information for each of the steps in the phase object ${ }^{8}$.

\footnotetext{
In its current form, the information in figure 25 is very detailed and may not be usable. However, it is only meant to illustrate a concept and future work can aim to provide high level enactment summaries. In other words, some filtering technique appears to be highly desirable but for now the current prototype merely accomplishes the essential, that is, logether the information to be subsequently filtered.
} 


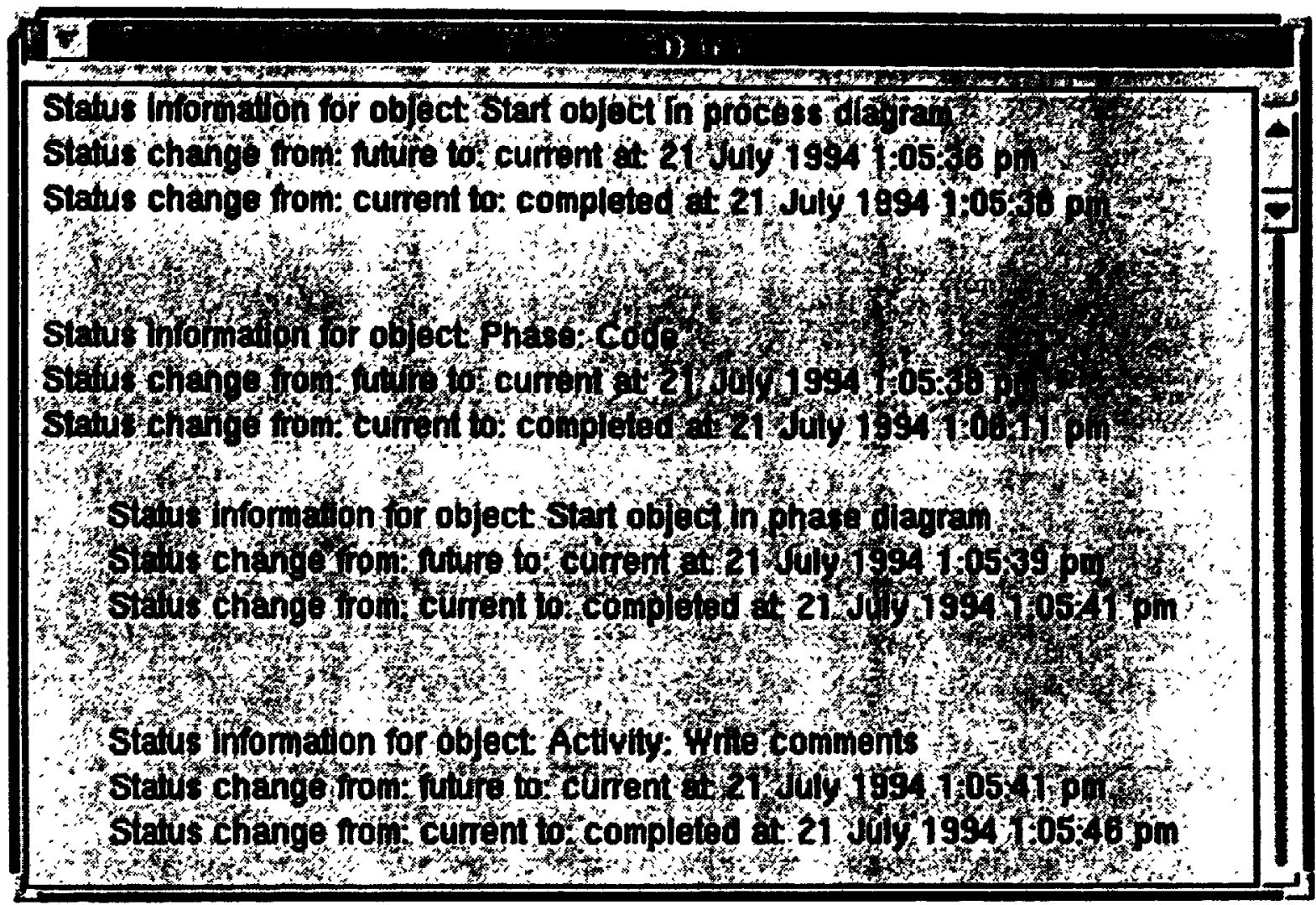

Figure 25: Enactment summary

This status information is one form of metric use in the process model. It records time information about the development process. The data can be used to see which parts of the process take the longest time. Efforts to improve productivity can then focus on these parts of the process. The other form of metric use occurs with GQMA objects. These are described in the next section.

\subsection{2) Metrics}

When a GQMA object is activated, the metric defined in the GQMA object is collected through the invocation of its associated Smalltalk method. More specifically, the method that was specified in the GQMA browser is sent to the class that was specified in the GQMA browser. The work package that is associated with the process 
model that contains the GQMA object is passed as an argument with the message. This allows the metric method to access the applications and classes within the applications that were defined as relevant to the work package. It is on these applications and classes that the metrics are collected. For example, a metric to measure the number of methods in a class will collect the metric for each class contained in the relevant applications associated with the work package. The metric results are stored in ENVY and are accessible through the process model. By selecting a GQMA object, the metric results that were collected can be viewed. This is shown in figure 26 .

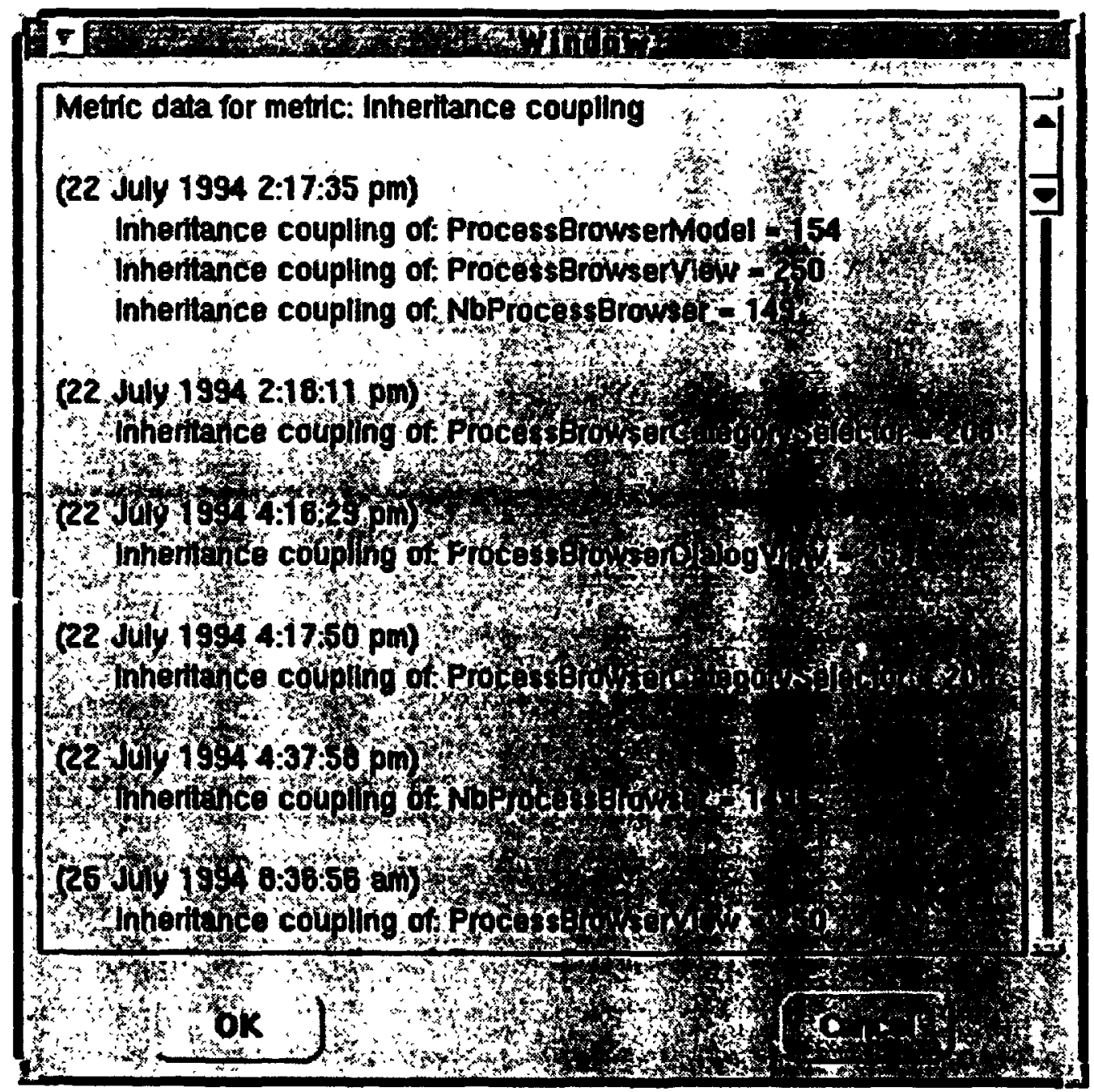

Figure 26: Metric results 
Each time the GQMA object is enacted, the time and date are recorded and the metric is collected. The results window displays each time-stamped metric result for the selected GQMA object. In figure 26, the inheritance coupling metric's is displayed. Each time stamp contains metric values for the classes that the metric was collected on.

The results window allows the results of a metric to be viewed in the context of the process model it was collected in. It is the simplest way in which to view metric data because it does not allow comparisons with other metric data9.

Vision has two ways in which metric data for the entire project can be compared. The first one organizes metrics by work packages. Using a browser like interface, projects and their categorized work packages can be viewed. Each work package has a list of the metrics and metrics data that were collected in the process models associated with the work package. Through this interface, metrics data can be selected for analysis. This organization method allows metric data for work packages to be analyzed. It can be used to show differences between work packages and trends in the project. This is the type of analysis that managers would perform. For example, a histogram showing the test coverage for all work packages in a project category can be used to find the work packages with the lowest test coverage. Another example would be to calculate the average defect density for each project category. This can show if the defect density is rising or falling as the project progresses.

The second method organizes metrics by ENVY applications. Recall that each work package stores a list of relevant applications. These applications contain the classes which implement the features of the system that is being developed. Having them referenced to work packages allows the tasks that specify the work to be traced to the

9Again, the information presented is verbose and should be summarized. 
actual code written. Through this mechanism, the metrics collected in the process model describing how to perform the task are linked to the actual code they measure. Therefore, by viewing the applications, it is possible to view any metrics that were collected on a given application. Using another browser like interface, the applications and associated metric data can be displayed and selected for analysis. This organization can be used to show differences between applications and classes that were developed. This is the type of analysis that developers would perform. For example, a developer can compare the test coverage for a class they developed to the project average. This gives the developer a relative indication of how well they tested their class.

From data selected through either of the two interfaces, Vision can calculate the mean, median, mode, and standard deviation. It can also draw a histogram, scatter plot, and multiple metrics graph. These analysis techniques allow the metric data to answer their questions and benefit the project.

\section{9) Usage scenarios}

In the following sections, the envisioned manner in which Vision is to be used on a project is presented.

\subsection{1) Library supervisor}

One of Vision's strengths is that it allows libraries of process models and GQMA objects to be built up. The person designated as the library supervisor will be responsible for maintaining these libraries. This person will use the process editor and GQMA browser to create process models and GQMA objects. The library supervisor may also 
receive process models and GQMA objects that were created by individual developers who saw an opportunity to improve the existing ones. These tried and tested models and metrics will be placed in the global process browser and global GQMA browser. The global browsers will therefore contain the process models and GQMA objects that are used on the project.

The process models placed in the global process browser may take two forms generic or project specific. Generic process models typically will not contain many GQMA objects. They will just state a generic development process. The goal is to allow generic process models to be used on any project. Project specific process models represent a specific process that was (or will be) used on a specific project. These models will include metrics that are used to address specific aspects of the project.

\subsection{2) Manager}

A manager of a project will look to the global process browser to find the process models that are applicable to their current project. If the current project is similar to a past project, the manager can use the project specific process model for that project. This will contain the steps and metrics that are needed for the current project. If no such project was done before, the manager will have to use a generic process model. This will give the process to follow. Depending of the project's goals, the manager will add the appropriate GQMA objects to the generic process model. This will make the process model project specific since it now uses metrics to address the goals of the current project. 
Once the manager has the correct process model, he or she will use the managers interface to create a new project object. This will hold the work packages for the current project. Since Vision is used in object oriented projects, the project will be divided into many iterations that each result in the development of a small part of the system. Vision uses categories to represent these iterations. The manager creates a category to hold the tasks of the first cycle. In this category, the manager creates work packages that represent the tasks to be completed in the cycle. The process model that represents the software development process telling how to do the work package is associated with each work package. Finally, the work packages are assigned to the developers who are responsible for completing them.

During the cycle, the manager can view the process models associated with a work package to determine which step the developer is performing. Daily or weekly reports can be generated which summarize the status of each work package. Additionally, the metrics associated with the work packages can be analyzed. For example, the manager can look at all work packages and pick out the five with the lowest value for the test coverage metric. Using these techniques, the managers can use Vision to effectively track and control the project.

\subsection{3) Developer}

The developer uses the developers interface to see which work packages they are responsible for. For a given work package, they enact the process models associated with the work package. The developer keeps the process model up to date as to which activity they are currently working on. This guides the developer on following the process as well as communicating their progress to others. 
As the model is enacted, any specified metrics are collected. The developers can use the results to get an indication of the quality of the work they did in the work package. This allows developers to use Vision to help them perform their job and make sure the work they do is of high quality.

During the project, the developer may think of ways to improve the current process or come up with different metrics to collect. They can use the process editor or GQMA browser to create new models and metrics. These can be stored in their local process browser and local GQMA browser. The developer can try out the new process model or metrics by themselves. Once they are sure they provide a benefit, they can give them to the library supervisor to place into the global browsers. This allows any developer to suggest process and metric improvements.

\subsection{0) Benefits}

This concludes the description of Vision's features and its intended usage. In this section, the benefits of Vision will be discussed.

Process ccmmunication. By providing an on-line graphical representation of the development process, each developer has a clear understanding of how to follow the process. This helps with training new developers as to how software is developed at an organization. It is also useful in helping existing developers leam a new process. This situation would occur if the organization is changing from the structured paradigm to the object oriented paradigm. In this case, Vision is used to help ease the transition by providing process guidance. 
Process improvement. With the process clear to everybody, there is a greater chance of people noticing inefficiencies in the process. Vision offers an easy way to make process modifications and try out the new process. If the new process is indeed better than the old one, it can be adopted as the standard development process. This is an example of how Vision supports continuous process evolution and improvement.

Process library. Vision's process library allows process models to be stored for reuse. By using Vision over several different types of projects, a library of project specific process models can be built up. This library will contain the appropriate process to use for a given type of project. Some examples are, process models to support the development of prototypes, research systems, or commercial products. This allows an organization to reuse and apply its past experience on current projects.

Tracking. By associating process models with work packages, Vision allows the tasks of a project to be tracked at the process level. Moreover, since Vision automatically records the time spent in each process step, reports of the project's time distribution can be automatically generated. This means that developers do not have to fill out time sheets and managers get immediate information as to each developer's status.

Metric collection. Vision provides a framework to define, store, and reuse metrics. Since the metrics are integrated with the process model, they are automatically collected as a consequence of enacting the model. Furthermore, because the process model is followed by each developer, the metrics are consistently collected in a project and across different projects. This allows the metrics results to be accurately compared with each other. 
Metric analysis. By storing the goal, question, and analysis technique with a metric, metrics become more than just numbers. GQMA objects make it clear as to what the metrics are measuring and how they can be used to benefit the project. Since the metrics are collected in the development environment, they can be immediately used to help the project. Vision also recognizes that managers and developers have different uses for metrics and gives different analysis techniques for each of them. Finally, Vision stores a historical record of all metrics results for a project. This provides data for project audits and post mortem analysis. 


\section{6) Future work}

There are two streams of research that can be started from this thesis. The first is to make improvements to Vision. Vision's implementation can be augmented with more features to help users productively use the tool. Some examples of these are providing more sophisticated metrics analysis techniques, making the analysis of metrics more automated (instead of just a text description of what to do), allowing work packages to be hierarchically nested for management of large numbers of work packages, or linking Vision to analysis and design CASE tools to provide better coverage across the software lifecycle.

The second form of research would be to use Vision to study software development projects. By modeling different object oriented methodologies, the relative advantages and disadvantages of each can be determined. Analyzing the actual process models that were used in a project can lead to a better understanding of what happens from the start to the finish of object oriented projects. Finally, Vision can be used to study the validity of current object oriented metrics and to determine new metrics and assess their usefulness. 


\section{7) Conclusion}

The purpose of this thesis was to develop a framework to assist object oriented software development. The framework was realized in Vision to show its validity and usefulness. Vision draws from the areas of object oriented software development, project management, process modeling, and metrics. The end result is an environment to develop, manage, and track an object oriented software development project.

Vision fills an important gap by providing automated support for the tracking of a software project. This is an area that has been somewhat neglected because management is not traditionally viewed as a computer science topic. Therefore, providing an integrated environment in which to develop and track the development is an important step to ensuring the success of a project. The management of the project is further supported through the use of process modeling and metrics.

The significance of Vision is that it is completely integrated with the Smalltalk and ENVY/Developer environment. Alone, the Smaltalk environment provides excellent features to support the development of software. ENVY/Developer augments this environment with support for multi-developer teams and large cross platform development efforts. The next logical step was to add tools to support the management of a software development project. Vision provides this through its process modeling and metrics capabilities. As a result, projects should be more accurately tracked, measured, and managed. Vision gives more power to managers and developers in their struggle against the software crisis. 


\section{8) References}

Andersen, O. (1992) Industrial applications of software measurements, Information and Software Technology, Vol. 34, No. 10, p. 681-693

Basili, V. R. and Rombach, H. D. (1987) Tailoring the software process to project goals and environments, Proceedings of the 13th International Conference on Software Engineering, p. 345-357

Basili, V. R. and Rombach. H. D. (1988) The TAME project: Towards improvementoriented software environments, I.E.E.E. Transactions on Software Engineering, Vol. 14, No. 6, p. 758-773

Bochm, B. W. (1986) A Spiral Model of Software Development and Enactment, Suftware Engineering Notes, Vol. 11, No. 4

Booch, G. (1991) Object Oriented Design with Applications, Benjamin Cummings Publishing, Menlo Park, CA.

Booch, G. (1994) Object Oriented Analysis and Design with Applications, Benjamin Cummings, Publishing, Menlo Park, CA.

Budd, T. (1991) Object-Oriented Programming, Addison-Wesley, Reading, MA. 
Chapin, N. (1988) Software Maintenance Life Cycle, 1988 I.E.E.E. Conference on Software Maintenance, p. 6-13

Chen, J. Y. and Lu, J. F. (1993) A new metric for object-oriented design, Information and Software Technology, Vol. 35, No. 4, p. 232-240

Chidamber, S. R. and Kemerer C. F. (1991) Towards a metrics suite for object oriented design, OOPSLA ‘9/, p. 197-211

Conte, S. D., Dunsmore, H. E., and Shen, V. Y. (1986) Software Engineering Metrics and Models, Benjamin Cummings Publishing, Menlo Park, CA.

Coppick, J. C. and Cheatham, T. J. (1992) Software metrics for object-oriented systems, CSC ‘92 - 20th Annual Computer Science Conference, p. 317-322

Curtis, B., Kellner, M. I., and Over, J. (1992) Process modeling, Communications of the $A C M$, Vol. 35, No. 9, p. 75-90

Dale, C. J. and van der Zee, H. (1992) Software productivity metrics: who needs them?, Information and Software Technology, Vol. 34, No. 11

deChampeaux, D., Lea, D., and Faure, P. (1993) Object-Oriented System Development, Addison Wesley, Reading, MA.

deMarco, T. (1982) Controlling Software Projects - Measurement, Management, \& Estimation, Yourdon Press, Prentice Hall, Englewood Cliffs, NJ. 
Evangelist, M. (1988) Complete solution to the measurement problem, I.E.E.E. Software, January, p. 83-84

Factor, R. M. and Smith, W. B. (1988) A discipline for improving software productivity, AT\&T Technical Journal, July/August, p. 2-9

Fenton, N. E. (1991) Software Metrics: A Rigorous Approach, Chapman and Hall, London, UK.

Fernström, C. (1993) Process weaver: Adding process support to UNIX, Proceedings of the 2nd International Conference on Software Process Modeling, p. 12-26

Fernström, C., Närfelt, K., and Ohlsson, L. (1992) Software factory principles, architecture, and experiments, I.E.E.E. Software, March, p. 36-44

Goldberg, A. and Robson, D. (1993) Smalltalk-80: The Language and its Implementation, Addison-Wesley, Reading, MA.

Grady, R. B. (1990) Work-product analysis: The philosopher's stone of software?, I.E.E.E. Software, March, p. 26-34

Grady, R. B. and Caswell, D. L. (1987) Software Metrics: Establishing a Company-Wide Program, Prentice-Hall, Englewood Cliffs, NJ.

Haaland, K. G. (iפ9'2) Towards metrics for object oriented design, Unpublished masters thesis - Carleton University 
Hall, P. A. V. (1992) Overview of Reverse Engineering and Reuse Research, Information and Software Technology, Vol. 34, No. 4, p. 239-249

Henry, S. M. and Humphrey, M. (1990) A Controlled Experiment to Evaluate Maintainability of Object Oriented Software, 1990 I.E.E.E. Conference on Software Maintenance, p. 258-265

Høydalsvik G. M. and Sindre. G. (1993) On the Purpose of Object-Oriented Analysis, OOPSLA ‘93, p. 240-255

Iida, H., Mimura, K., Inoue, K., and Torii, K. (1993) Hakoniwa: Monitor and navigation system for cooperative development based on activity sequence model, Proceedings of the 2nd International Conference on the Software Process, p. 64-74

Ince, D. (1990) Software metrics: introduction, Information and Software Technology, Vol. 32, No. 4, p. 297-303

Inglls, J. (1986) Standard software quality metrics, AT\&T Technical Joumal, Vol. 65, No. 2, p. 113-118

Jacobson, I. et al. (1992) Object-oriented Software Engineering, Addison-Wesley, Reading, MA.

Kitchenham, B. and Pickard, L. (1987) Towards a constructive quality model, Software Engineering Journal, July, p. 114-126 
Kolewe, R. (1993) Metrics in object-oriented design and programming, Software Development, October, p. 53-61

Krasner, H., Terrel. J., Linehan, A., Arnold, P., and Ett, W. H. (1992) Lessons learned from a software process modeling system, Communications of the ACM, Vol. 35, No. 9 , p. $91-99$

Li, W. and Henry, S. (1993) Object-oriented metrics that predict maintainability, Journal of Systems Software, No. 23, p. 111-122

Lientz, B. P. and Swanson, E. B. (1981) Problems in Application Software Maintenance, Communications of the ACM, Vol. 24, No. 11, p. 764-769

Lorenz, M. and Kidd, J. (1994) Object-Oriented Software Metrics, Prentice Hall, Englewood Cliffs, NJ.

Meyer B. (1988) Object Oriented Software Construction, Prentice Hall, Englewood Cliffs, NJ.

Meyer, B. and Nerson, J. M. (1993) Object-Oriented System Development, Prentice Hall, Englewood Cliffs, NJ.

Mi, P. and Scacchi, W. (1992) Process integration in CASE environments, I.E.E.E. Software, March, p. 45-53

Monarchi, D. E. and Puhr, G. I. (1992) A Research Typology for Object Oriented Analysis and Design, Communications of the ACM, Vol. 35, No. 9, p. 35-47 
Myers, W. (1989) Allow plenty of time for large-scale software, I.E.E.E. Software, July, p. $92-99$

Myrvold, A. (1990) Data analysis for software metrics, Joumal of systems software, No. 12, p. $271-275$

Notkin, D. (1988) The relationship between software development environments and the software process, SIGPLAN Norices, Vol. 24, No. 2, p. 107-109

Penedo, M. H. and Shu, C. (1991) Acquiring experiences with the modeling and implementation of the project life-cycle process: the PMDB work, Sofnware Engineering Journal, September, p. 259-274

Pfleeger, S. L. (1993) Lessons learned in building a corporate metrics program, I.E.E.E. Sofnvare, May, p. 67-74

Pfleeger, S. L., Fitzgerald Jr., J. C., and Rippy, D. A. (1992) Using multiple metrics for analysis of improvement, Software Quality Journal, No. 1, p. 27-36

Pittman, M. (1993) Lessons learned in managing object oriented development, I.E.E.E. Software, January, p. 43-53

Reuter, V. G. (1979) Using graphic management tools, Joumal of Systems Management, April, p. 6-17 
Robertson, L. B. and Secor, G. A. (1986) Effective management of software development, AT\&T Technical Journal, March/April. p. 94-101

Rosson, M. B. and Alpert, S. R. (1990) The Cognitive Consequences of Object-Oriented Design, Human-Computer Interaction, Vol. 5, p. 345-379

Selby, R. W., Porter, A. A., Schmidt, D. C., and Berney, J. (1991) Metric-driven analysis and feedback systems for enabling empirically guided software development, $13 \mathrm{th}$ International Conference on Software Engineering, p. 288-298

Shepard, T., Sibbald, S., and Wortley, C. (1992) A visual software process language, Communications of the ACM, Vol. 35, No. 4, p. 36-44

Shepperd, M. (1992) Products, processes and metrics, Information and Software Technology, Vol. 32, No. 10, p. 674-680

Sommerville, I. (1992) Sofnvare Engineering, Addison-Wesley, Reading, MA.

Valett, J. D. and McGarry, F. E. (1989) A summary of software measurement experiences in the software engineering laboratory, Journal of Systems Software, No. 9, p. $127-148$

Wilkie, G. (1993) Object-oriented software engineering: The professional developer's guide, Addison-Wesley, Reading, MA.

Wirfs-Brock, R., Wilkerson, B., and Wiener, L. (1990) Designing Object Oriented Software, Prentice Hall, Englewood Cliffs, NJ. 
Zells, L. (1990) Managing Software Projects: Selecting and Using PC-Based Project Management Systems, QED Information Sciences Inc. 


\section{Appendix 1) ENVY/Developer}

In this appendix, ENVY/Developer (from now on referred to as ENVY) is briefly described. For a more thorough presentation on the subject, the ENVY customer documentation should be referenced.

ENVY is a source code management system. It is implemented in Smalltalk and forms an integrated part of the development environment. By itself, the base Smalttalk environment contains all the necessary tools to support single user development. ENVY adds support for multi-developer teams, cross-platform development, and configuration management.

The main part of ENVY is the shared library. It stores all of the software components (methods, classes, class extensions, applications, and configuration maps) that were created by the users of ENVY. Each of these users has their own private image in which they develop. Whenever a user defines a component, it is immediately stored in their local image and the shared library. In order to use components in the shared library, the other users must explicitly load the components from the shared library to their local images. This is how code sharing occurs. A user potentially has access to another user's changes but has control over whether or not to use them.

ENVY augments the base Smalltalk development objects (classes and methods) to include configuration maps, applications, and class extensions. Applications group together related classes. Collectively the classes in an application serve a useful purpose. Applications therefore support reuse of collections of classes. This provides a larger granularity of reuse than classes alone. Applications can be arranged in a sub-application 
hierarchy and can have other applications as pre-requisites. The pre-requisite relationship requires other applications to be present for a given application to function.

Basic Smalltalk classes are defined in one application. Another application can define a class extension of a class. This allows the other application to add functionality to the class that is specific itself. Unless the other application is loaded into the image, the extension will not be visible.

Configuration maps group applications into a system. A configuration map specifies which applications to load. By having different configuration maps, different versions and releases of the final system can be loaded.

Each software component (methods, classes, class extensions, applications, and configuration maps) can be made an edition, version, or scratch. An edition is an editable component. While a component is being developed, it is an edition. Once the compenent is in a stable state, it is versioned. A versioned component, cannot be changed. Versioning provides a way to freeze changes and create a baseline. After a component has been versioned, it can be released. This makes it available for other users to load the component from the shared library to their local image. A scratch of a component is a private edition. Scratch components cannot be released. They provide a way for users to do experimentations that they do not want anybody else to have.

Within ENVY, there are specific roles and ownership rights. For example, each class has an owner. The owner is the only one who can release the class. The class can have many developers associated with it. Developers can make editions of the class but these changes must be integrated with the class owner before the class is released. Class ownership ensures that the originator of the class is responsible for maintaining the class' 
integrity by requiring the owner to approve any changes before the class is released Similar roles exist for applications and configuration maps.

Development using ENVY follows a cycle of creating editions, developing, versioning, releasing, and integrating. In each cycle, a portion of the functionality of the system being developed is added. To begin, developers create editions of the necessary components so they can edit them. A developer then develops the classes they were assigned to do. Once the developer is done, the classes are versioned and released. This allows other developers integrate the released classes with their own work. Once all of the developers are finished, the classes and applications are versioned and released. A configuration map points to these versions so that each developer can load in a baseline of functionality. This cycle is repeated until the entire system is developed.

This concludes the discussion on ENYY. ENVY provides important features necessary for large scale object oriented software development. ENVY's main advantage over other source code management systems is that it is completely integrated with the Smalltalk development environment. This makes ENVY natural to use and reconcile with the incremental development style that is supported by Smalltalk. 


\section{Appendix 2) Example Process Model}

In this appendix, an example object oriented software development process is represented by Vision.

\section{The process}

The process consists of four phases which are continuously performed until the entire system that is being developed is completed. The purpose of each phase is given below.

Requirements - this phase identifies the requirements that will be implemented in a development iteration.

Analysis - this phase models the objects and interactions of objects that specify what the system does.

Construction - this phase refines the analysis objects into a design and implementation of the system.

Validation - this phase integrates and tests the code produced in the current iteration with the code that was produced in previous iterations.

The process diagram to represent these phases is shown in figure A-1. 


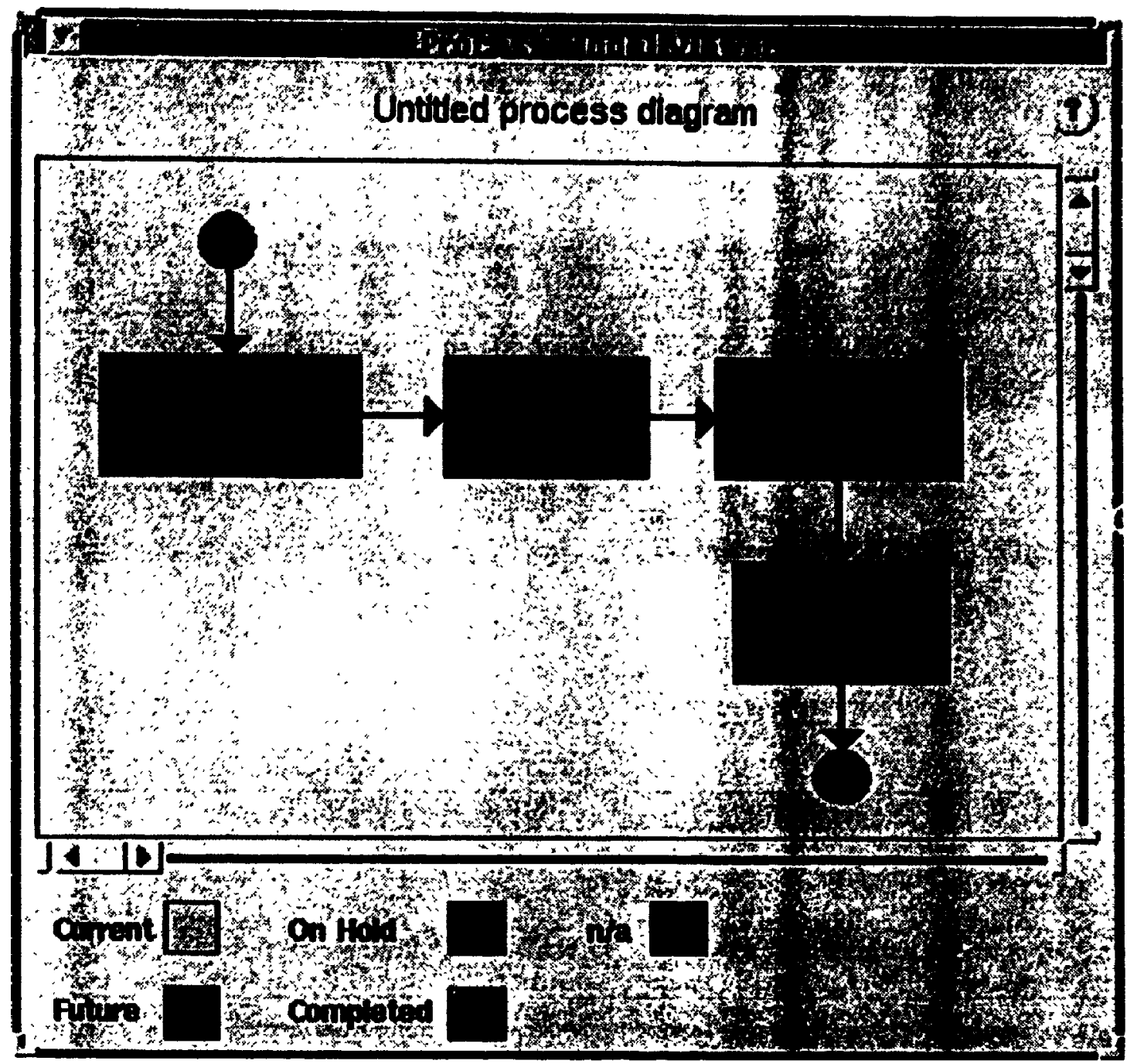

Figure A-1: Process diagram

\section{The phases}

In the following sections, each of the activities in the phases are describeo. 


\section{Requirements phase}

The activities in the requirements phase are described below. Vision's representation of the phase is shown in figure A-2.

Identify requirements - based on the previous iterations and the functional specification for the system, determine candidate requirements to implement this cycle.

Assign rank - give each requirement a composite ranking which considers the requirement's importance, difficulty, and feasibility. The highest ranking requirements will be done first, then if time is still available in the cycle the lower ranking requirements will be done.

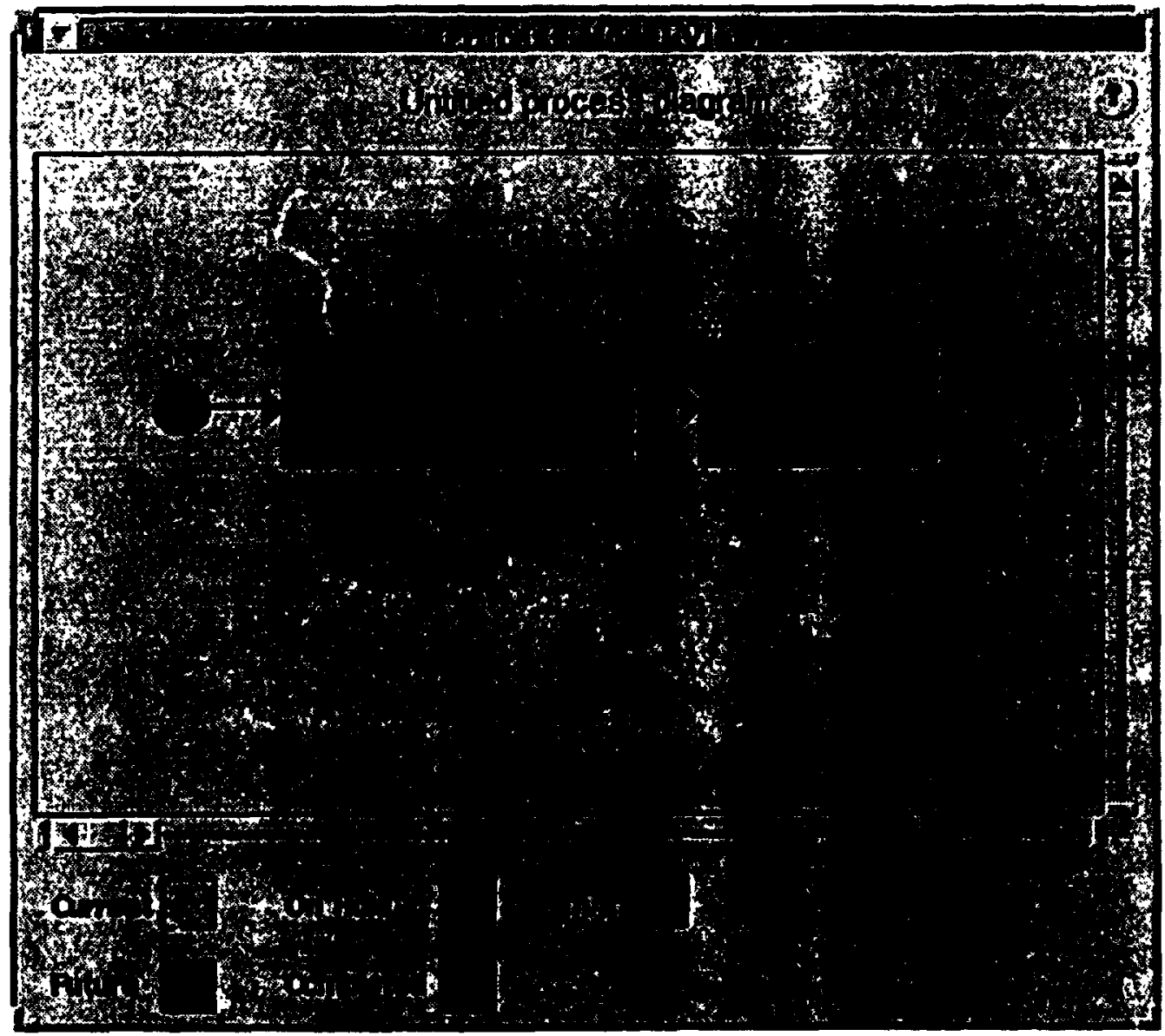

Figure A-2: Requirements phase 


\section{Analysis phase}

The activities in the analysis phase are described below. Vision's representation of the phase is shown in figure A-3.

Discover objects - based on the requirements, determine the objects necessary to represent the requirements and assign each object their responsibilities.

Model interactions - model the message interaction that occurs between the objects in order to realize the requirements.

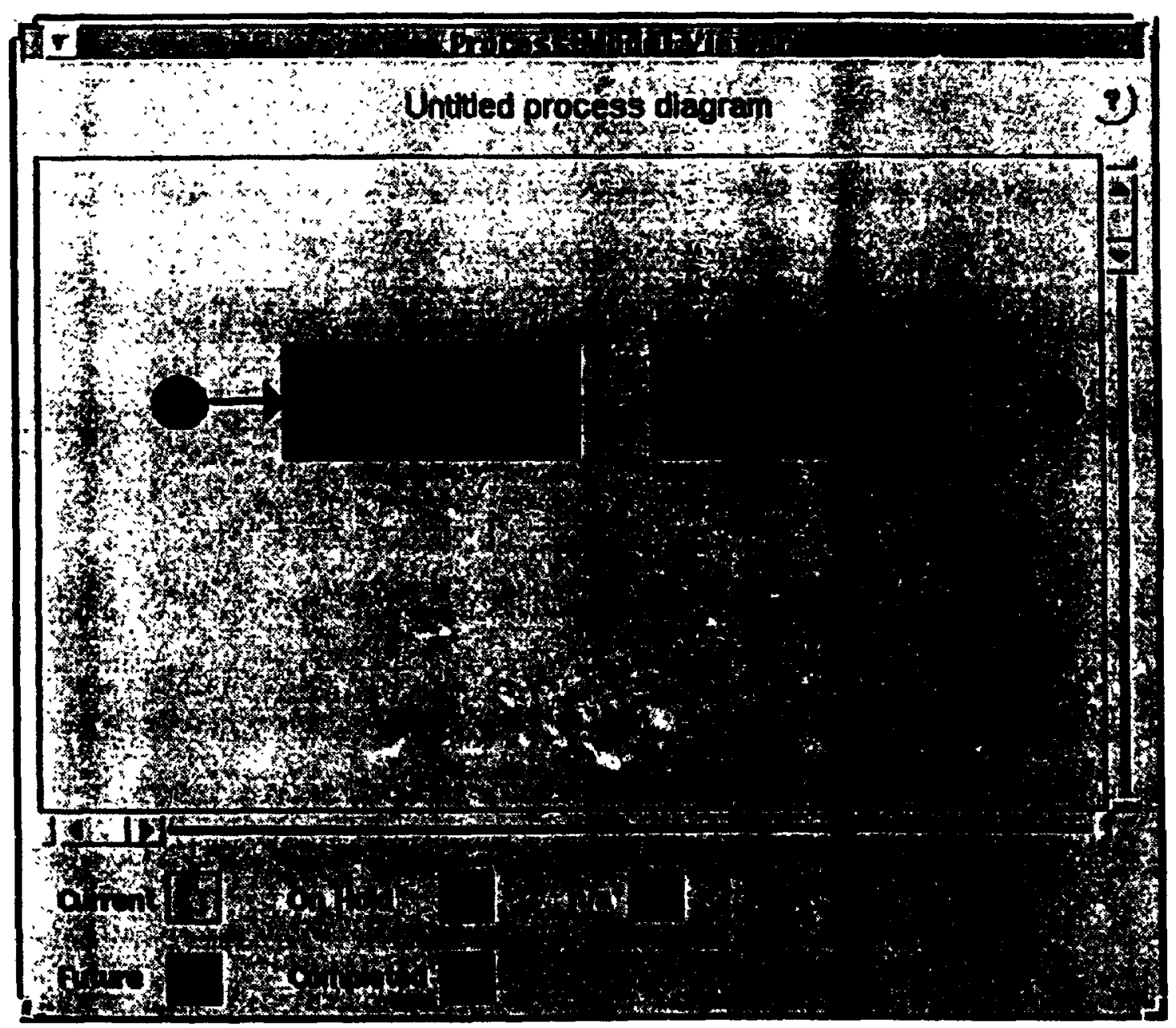

Figure A-3: Analysis phase 


\section{Construction phase}

The activities in the construction phase are described below. Vision's representation of the phase is shown in figure A-4.

Model design solution - refine the analysis model to account for design issues. The model should now contain design objects, the objects' interfaces, and the objects' use cases.

Implement - from the design model, implement the code to realize the design.

Write test cases - write test cases to test the implementation and ensure that the requirements have been met.

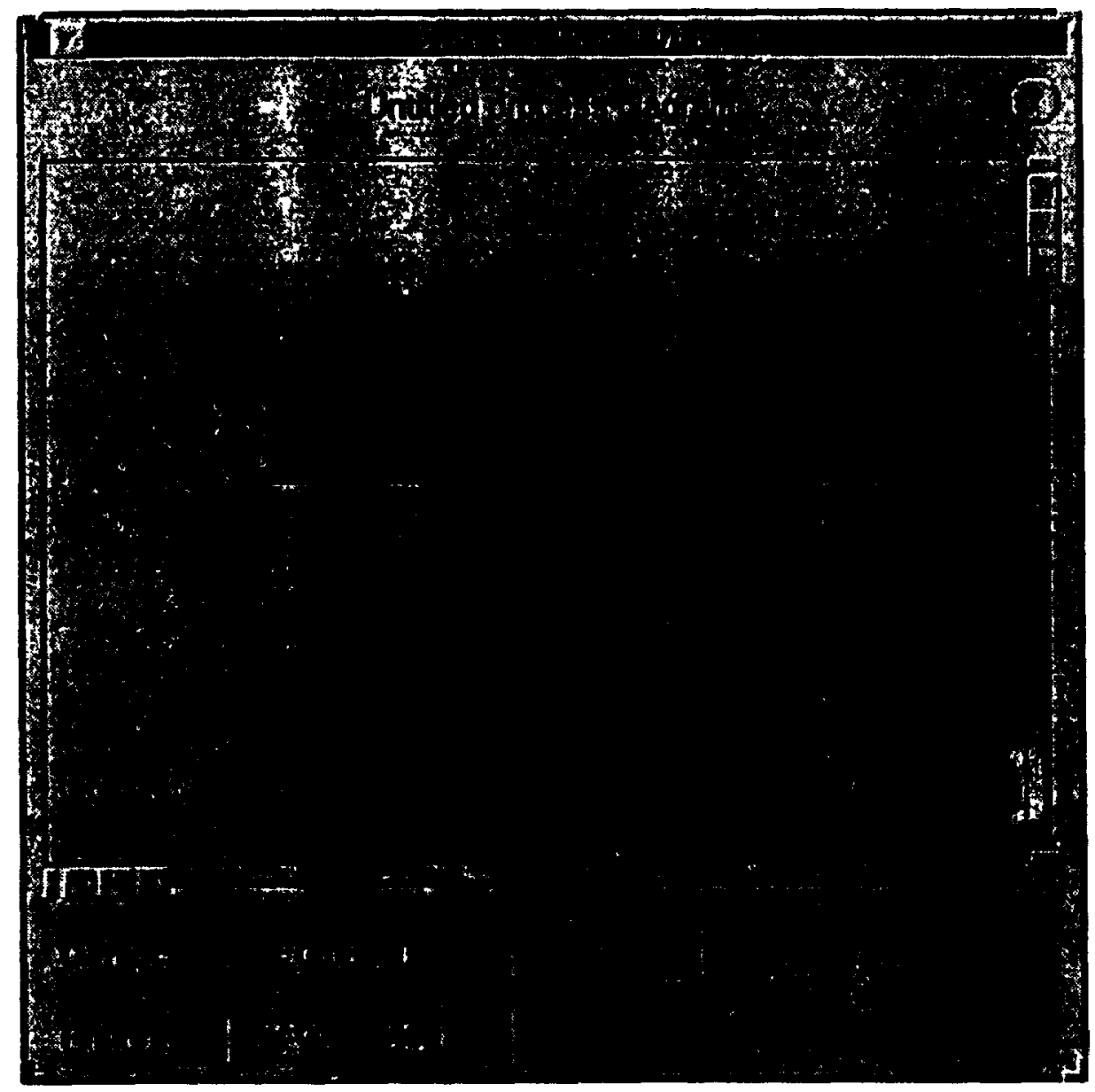

Figure A-4: Construction phase 


\section{Validation phase}

The activities in the validation phase are described below. Vision's representation of the phase is shown in figure A-5.

Integrate - integrate the code that was implemented in the current cycle with the rest of the system.

Test - run the test cases to make sure the new code works with the rest of the system.

Regression test - re-run the old test cases to make sure the old features of the system still work properly.

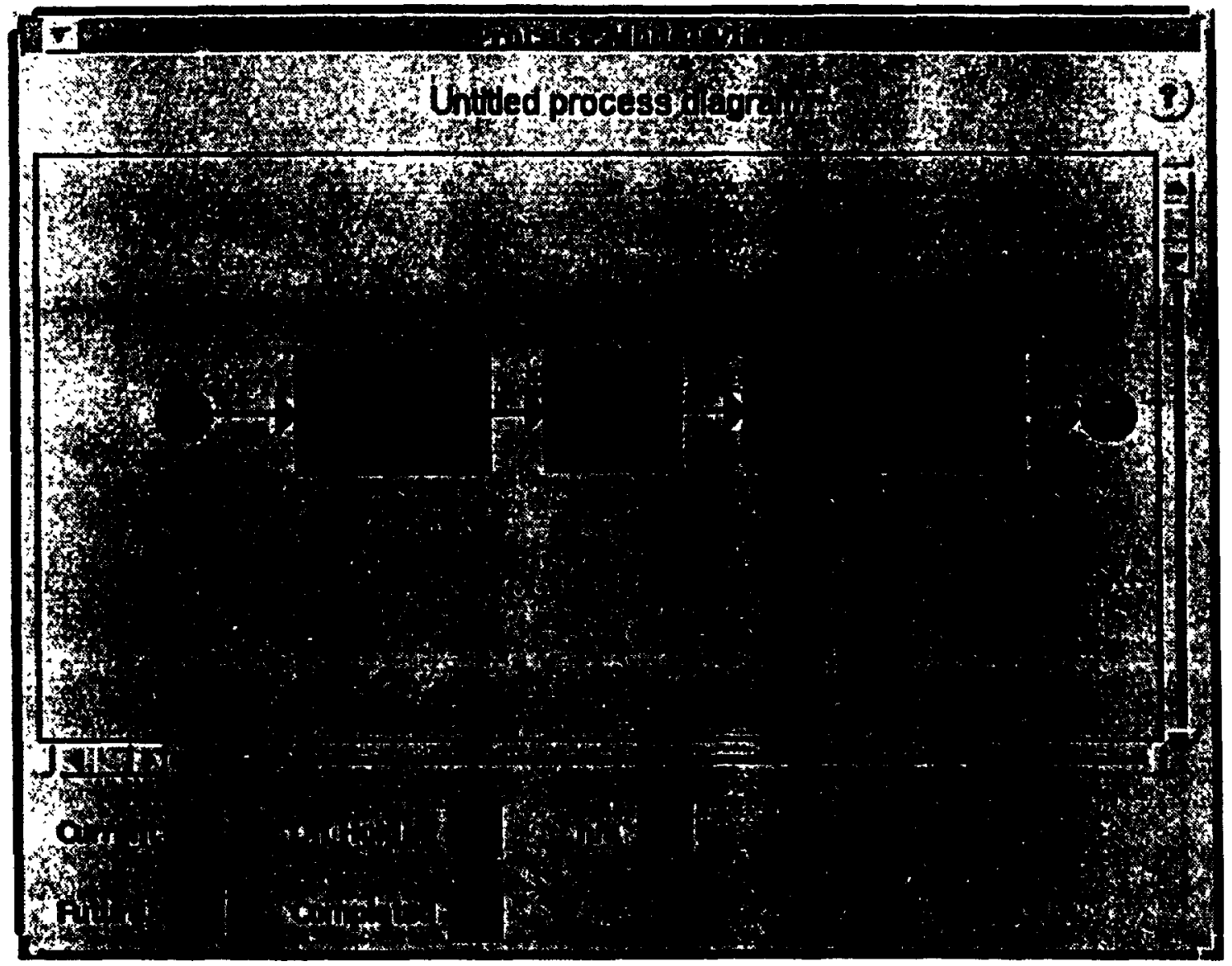

Figure A-S: Validation phase 


\section{Appendix 3) Implementation}

This appendix gives a brief overview of how Vision is implemented. The ENVY application hierarchy for the tool is as follows.

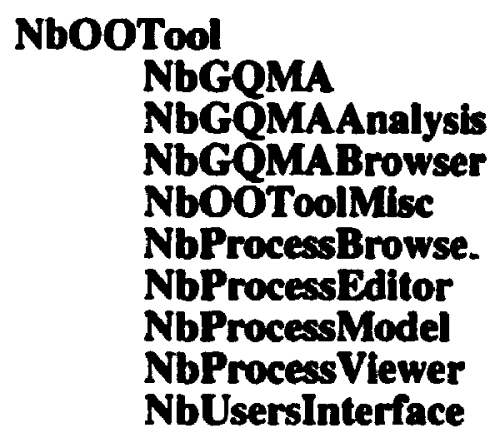

Each application's purpose and the classes it contains are given below.

Application: NbOOTool

Purpose: The root application to hold all sub-applications that implement the tool.

Classes: NbOOTool

Application: NbGQMA

Purpose: Implements the classes to collect and store metrics as well as represent the GQMA object.

Classes: $\quad$ GQMAObject, MetricCollection, MetricStorage, NbGQMA 
Application: NbGQMAAnalysis

Purpose: Implements the browsers to view and analyze metric data.

Classes: $\quad$ GQMAAnalysisBrowser, TaskGQMAAnalysisBrowser, NbGQMAAnalysis

Application: NbGQMABrowser

Purpose: Implements the local and global GQMA browsers.

Classes: GQMABrowserOrganizer, GQMABrowser,

GlobalGQMABrowser, GQMABrowserOpenDialog,

GlobalGQMABrowserOpenDialog, NbGQMABrowser

Application: NbOOToolMisc

Purpose: Contains miscellaneous windows used by all of the other applications (e.g. the on-line help window).

Classes: HelpInterface, SelectorInterface, TextWindowDialog,

TextWindowInterface, NbOOToolMisc

\section{Application: NbProcessBrowser}

Purpose: Implements the local and global process browsers.

Classes: BrowserOrganizer, ProcessBrowserOrganizer, ProcessBrowserControiler, ProcessBrowserDialogController,

ProcessBrowser, GlobalProcessBrowser,

ProcessBrowserCategorySelector, ProcessBrowserOpenDialog,

GlobalProcessBrowserOpenDialog, ProcessBrowserModel,

NbProcessBrowser, ProcessBrowserView,

ProcessBrowserDialogView 


\section{Appllication: NbProcessEditor}

Purpose: Implements the process editor.

Classes: ProcessEditorController, ProcessEditorSelectionDragMode, ProcessEditorSelectMode, ProcessEditor, ProcessEditorModel, NbProcessEditor, ProcessEditorView

Application: NbProcessModel

Purpose: Implements classes to represent the process model and its component process objects.

Classes: ActivityInformationInterface, DialogInformationInterface, GenericInformationInterface, GQMAInformationInterface, NameInformationInterface, PhaseInformationInterface, SynchrolnformationInterface, ProcessEditorObjectModel, LinkableObjectModel, ActivityModel, DialogModel, EndModel, PhaseEndModel, GenericModel, GQMAModel, PhaseModel, StartModel, PhaseStartModel, SynchroModel, LinkModel, ProcessEditorDiagram, PhaseDiagram, ProcessDiagram, NbProcessModel, ProcessEditorObjectView, LinkableObjectView, FixedSizeView, EndView, StartView, SynchroView, ToolDialogView, VariableSizeView, PolygonView, RectangleView, LinkView, ArrowedLineWrapper 


\section{Application: NbProcessViewer}

Purpose: Implements the process model viewer to view the enactment of a process model.

Classes: ProcessViewerController, Process ViewerManagerController, ChecklistInterface, ControlInterface, DataInterface,

ProcessModelViewer, ObjectSelectorInterface, Process ViewerModel, NbProcessViewer, ProcessViewerView, ProcessViewerManagerView

Application: NbUsersInterface

Purpose: Implements the developers and managers interfaces.

Classes: $\quad$ ProjectCreationInterface, WorkPackageCreationDialog, WorkPackageList, AbstractUsersInterface, DevelopersInterface, ManagersInterface, ProjectObject, NbUsersInterface, WorkPackage 

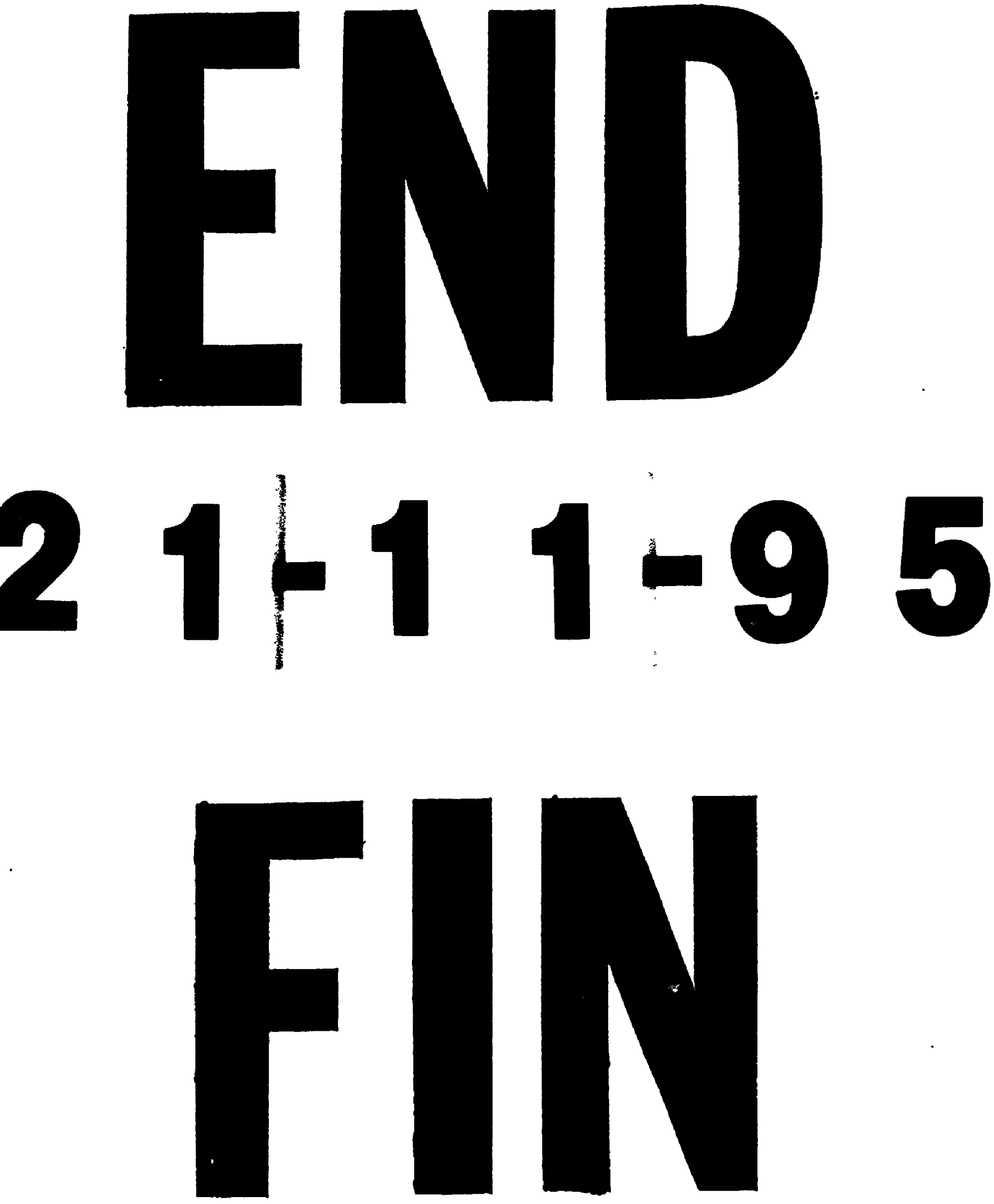